\title{
Das Selbstkonzept von Kindern in der Schuleingangsphase
}

\author{
Inauguraldissertation der Philosophisch-humanwissenschaftlichen
}

Fakultät der Universität Bern zur Erlangung der Doktorwürde

vorgelegt von

\section{Patrizia Cimeli}

Biberist/Winznau/Trogen (CH), San Marcello (IT)

Selbstverlag/Bern, Herbst 2012

Originaldokument gespeichert auf dem Webserver der Universitätsbibliothek Bern

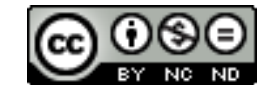

Dieses Werk ist unter einem

Creative Commons Namensnennung-Keine kommerzielle Nutzung-Keine Bearbeitung 2.5 Schweiz Lizenzvertrag

lizenziert. Um die Lizenz anzusehen, gehen Sie bitte zu http://creativecommons.org/licenses/by-nc-nd/2.5/ch/ oder schicken Sie einen Brief an Creative Commons, 171 Second Street, Suite 300, San Francisco, California 94105, 


\section{Urheberrechtlicher Hinweis}

Dieses Dokument steht unter einer Lizenz der Creative Commons Namensnennung-Keine kommerzielle NutzungKeine Bearbeitung 2.5 Schweiz. http://creativecommons.org/licenses/by-nc-nd/2.5/ch/

Sie dürfen:

(15)

dieses Werk vervielfältigen, verbreiten und öffentlich zugänglich machen

Zu den folgenden Bedingungen:

(1)

Namensnennung. Sie müssen den Namen des Autors/Rechteinhabers in der von ihm festgelegten Weise nennen (wodurch aber nicht der Eindruck entstehen darf, Sie oder die Nutzung des Werkes durch Sie würden entlohnt).

Keine kommerzielle Nutzung. Dieses Werk darf nicht für kommerzielle Zwecke verwendet werden.

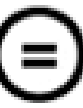

Keine Bearbeitung. Dieses Werk darf nicht bearbeitet oder in anderer Weise verändert werden.

Im Falle einer Verbreitung müssen Sie anderen die Lizenzbedingungen, unter welche dieses Werk fällt, mitteilen. Jede der vorgenannten Bedingungen kann aufgehoben werden, sofern Sie die Einwilligung des Rechteinhabers dazu erhalten.

Diese Lizenz lässt die Urheberpersönlichkeitsrechte nach Schweizer Recht unberührt.

Eine ausführliche Fassung des Lizenzvertrags befindet sich unter

http://creativecommons.org/licenses/by-nc-nd/2.5/ch/legalcode.de

Von der Philosophisch-humanwissenschaftlichen Fakultät der Universität Bern auf Antrag von Prof. Dr. Claudia M. Roebers (Hauptgutachterin) und Prof. Dr. Achim Conzelmann (Zweitgutachter) angenommen. 


\section{Danksagung}

Ich möchte mich bei allen herzlich bedanken, die mich während meinem Dissertationsprojekt unterstützt und begleitet haben.

Mein ausdrücklicher Dank gilt Frau Prof. Dr. C. Roebers, die es mir überhaupt erst ermöglicht hat, eine Dissertation zu schreiben. Ich hatte das grosse Glück, in ihr eine Betreuungsperson zur Seite zu haben, die mich in dieser Phase hilfreich begleitet, individuell gefördert und grosszügig unterstützt hat.

Meinen beiden Co-Projektleiterinnen, Dr. R. Neuenschwander und Dr. M. Röthlisberger danke ich für ihr grosses Engagement für unser Forschungsprojekt und für die motivierende und inspirierende Zusammenarbeit. Ebenso bedanke ich mich bei allen Lizentiats- bzw.

Masterstudierenden, Hilfskräften und Forschungspraktikantinnen, die bei der Datenerhebung und eingabe behilflich waren.

Ein spezieller Dank geht auch an alle ehemaligen und aktuellen Teammitglieder der Entwicklungspsychologie, wie auch an unsere Sekretärin L. Leuenberger. Besonders bedanken möchte ich mich bei Lic. phil. S. Fatzer, M. Sc. S. Loher und M. Sc. N. Destan für ihr kritisch-konstruktives Lektorat und für ihre humorvolle, emotionale Unterstützung.

Auch meiner Familie gebührt ein grosses Dankeschön. Bei meiner Schwester Manuela Cimeli möchte ich mich für das gründliche Lektorat und die unterstützenden Worte bedanken. Mein Ehemann Kaspar Schiess Cimeli war mir in dieser intensiven Phase ein unermüdlicher Diskussionspartner, hat wichtige Inputs eingebracht, mich ermutigt und mir die Freiräume eingeräumt, die ich nötig hatte, um die Dissertation zu einem Ende zu bringen - herzlichen Dank!

Nicht zuletzt gebührt mein Dank allen Lehrpersonen, Kindern und Eltern, die an unserem Forschungsprojekt teilgenommen haben und uns damit wertvolle Daten zur Verfügung gestellt haben und auch der Jacobs Stiftung Zürich, welche das Projekt mitfinanziert hat. 
Die vorliegende kumulative Dissertation umfasst folgende drei wissenschaftliche Studien zum Themengebiet „Das Selbstkonzept von Kindern in der Schuleingangsphase“:

\section{Studie 1}

Cimeli, P., Neuenschwander, R., Röthlisberger, M., \& Roebers, C. M. (im Druck). Das Selbstkonzept von Kindern in der Schuleingangsphase: Ausprägung und Struktur sowie Zusammenhänge mit frühen kognitiven Leistungsindikatoren. Zeitschrift für Entwicklungspsychologie und Pädagogische Psychologie.

\section{Studie 2}

Cimeli, P., Röthlisberger, M., Neuenschwander, R., \& Roebers, C. M. Stellt ein niedriges Selbstkonzept einen Risikofaktor für Anpassungsprobleme nach dem Schuleintritt dar? Zur Veröffentlichung eingereichtes Manuskript.

\section{Studie 3}

Roebers, C. M., Röthlisberger, M., Neuenschwander, R., \& Cimeli, P. School progress, academic performance, and self-regulatory processes as a function of early elementary school context. [Schulischer Werdegang, schulische Leistungen und selbst-regulatorische Prozesse in Abhängigkeit des Schuleingangsmodells] Manuscript resubmitted for publication. 


\section{Inhaltsverzeichnis}

Übersicht

1. Theoretischer Hintergrund und Fragestellungen der Dissertation

a) Selbstkonzept: Relevanz und Forschungsüberblick

b) Kontextualisierung der Dissertation

c) Validierung des Konstrukts Selbstkonzept (Fragestellung 1)

d) Selbstkonzeptgenese: Vergleichsprozesse (Fragestellung 2)

e) Modell zur Entwicklung des Selbstkonzepts (Ergänzung zur Fragestellung 1 und 2, Fragestellung 3)

2. Forschungsprojekt FIPS

a) Hintergrundinformationen zur Längsschnittstudie

b) Methodische Einbettung der drei Studien

c) Messinstrument zur Erfassung des Selbstkonzepts

3. Zusammenfassung der drei Studien

4. Diskussion der Fragestellungen

5. Zusammenfassung und Schlussfazit

Literaturverzeichnis

Anhang

Selbständigkeitserklärung 


\section{Übersicht}

Die vorliegende Arbeit dokumentiert die kumulative Dissertation, deren Kernstück die drei zur Veröffentlichung eingereichten Studien darstellen. Diesem zentralen Teil vorangestellt ist eine Erläuterung, die folgende fünf Teile beinhaltet: 1. den theoretischen Hintergrund, die Kontextualisierung und die drei übergeordneten Fragestellungen der Dissertation, 2. eine Vorstellung des Forschungsprojekts, aus welchem die Daten der Dissertation stammen, 3. eine Zusammenfassung der drei Studien, 4. die Diskussion der Fragestellungen und, 5. eine Zusammenfassung mit Schlussfazit.

\section{Theoretischer Hintergrund und Fragestellungen der Dissertation}

\section{a) Selbstkonzept: Relevanz und Forschungsüberblick}

Die Faszination am Selbst begleitet den Menschen schon sehr lange und erste Theorien zum Selbstkonzept entstammen den Anfängen der Psychologie als Wissenschaftsdisziplin. Seit Ende des 19. Jahrhunderts hat James (1890/1950) die Vorstellung eines dichotomen Ichs geprägt, das sich aus Subjekt („I“) und Objekt („Me“) zusammensetzt. Während dabei das „I“ der Akteur der eigenen Handlungen und des eigenen Wissens ist, wird das „Me“ als Produkt des Akteurs betrachtet und entspricht der gegenwärtigen Auffassung des Selbstkonzepts. William James wird deshalb von vielen als Begründer der Selbstkonzeptforschung angesehen. Während die Forschung zum Selbstkonzept in der Phase des Behaviorismus nur zögerliche Fortschritte machte, erwachte sie nach der kognitiven Wende erneut zum Leben (Übersicht bei Marsh, Debus, \& Bornholt, 2005). Heute stellt das Selbstkonzept ein wichtiges Konstrukt der psychologischen Forschung dar. Von der Bedeutung des Selbstkonzepts zeugen verschiedene Forschungsergebnisse, die aufzeigen, dass das Selbstkonzept dem Einfluss verschiedener interner und externer Faktoren unterliegt und sich gleichzeitig in hohem Masse auf das menschliche Verhalten auswirkt (z. B. Harter, 2012). 
Das Selbstkonzept wird von verschiedenen Teildisziplinen der Psychologie erforscht, z. B. der Persönlichkeitspsychologie, der Entwicklungspsychologie, der Pädagogischen Psychologie und der Klinischen Psychologie. Dabei lassen sich nach Krapp (1997) grob zwei Ansätze unterscheiden: der differenzielle Forschungszugang, welcher interindividuelle Merkmalsunterschiede zum Gegenstand hat und die prozessorientierte Forschungsperspektive, welche den dynamischen Charakter des Selbst untersucht.

Die historische Entwicklung der Forschung zum Selbst und die verschiedenen Forschungsansätze haben zu einer Heterogenität an Definitionen und theoretischen Modellen zum Selbst geführt (z. B. Moschner, 2001). Es lassen sich dabei drei Begrifflichkeiten theoretisch voneinander abgrenzen: Selbstkonzept (engl. self-concept, self-description, selfrepresentations, self-perceptions), Selbstwert (engl. self-worth, self-esteem) und Selbstwirksamkeit (engl. self-efficacy). Beim Selbstkonzept, dem subjektiven Bild der eigenen Person, steht die deklarative Komponente im Vordergrund (Helmke, 1992). Unter Selbstwert versteht man hingegen die affektiv-evaluative Komponente, die Bewertung dieses Selbstbilds (Asendorpf, 2012; Schütz, 2003). Selbstwirksamkeit wiederum ist ein von Bandura (1977) geprägter Begriff, der als Selbsteinschätzung einer Person aufgrund eigener Kompetenzen eine zukünftige Handlung erfolgreich ausführen zu können, definiert wird.

\section{b) Kontextualisierung der Dissertation}

Die vorliegende Dissertation zum Selbstkonzept von Kindern in der Schuleingangsphase lässt sich zwischen den Forschungsdisziplinen der Entwicklungspsychologie und der Pädagogischen Psychologie verorten und verfolgt überwiegend einen differenziellen Forschungsansatz. In diesen Forschungsdisziplinen stellt das Selbstkonzept einen wichtigen Eckpfeiler der sozio-emotionalen, kindlichen Entwicklung dar (z. B. Davis-Kean \& Sandler, 2001), dem in vielen Lebensbereichen eine wichtige verhaltensregulative Funktion zukommt (Roebers, 2007). Als Beispiel sei hier der ResilienzVulnerabilitäts-Ansatz anzuführen, der sich mit der potentiell entwicklungsförderlichen 
bzw. -hinderlichen Auswirkung eines hohen bzw. niedrigen Selbstkonzept beschäftigt (Scheithauer \& Petermann, 1999; Werner, 2007). Konkret lässt sich empirisch insbesondere die positive Auswirkung eines hohen leistungsbezogenen, akademischen Selbstkonzepts auf das Lernen nachweisen (bei Kindern am Ende der Grundschule, vgl. Helmke, 1992). Das akademische Selbstkonzept gilt dadurch als einflussreichstes nicht-kognitives Schülermerkmal, dem „eine Schlüsselrolle für die subjektive Bewältigung und Bewertung schulischer Lern- und Leistungssituationen zukommt“" (Helmke, 1998, S. 117). Die Ursprünge verschiedener Aspekte des Selbstkonzepts, die speziell für den schulischen Kontext eine Rolle spielen, werden im Vorschul- und frühen Grundschulalter, der sogenannten Schuleingangsphase, vermutet (Filipp, 2006; Harter, 1998; Marsh, Ellis, \& Craven, 2002). Genau für diese Altersspanne liegt aber gerade im deutschsprachigen Raum klar eine Forschungslücke vor, zur deren Schliessung die vorliegende Dissertation einen Teil beitragen soll.

Die Dissertation bezieht sich auf die Definition von Harter (1998), welche das Selbstkonzept als kognitive Konstruktion und mentale Repräsentation der eigenen Person definierte. Theoretisch orientiert sich unsere Forschung am Selbstkonzeptmodell von Shavelson, Hubner und Stanton (1976). Da wir uns jedoch mit der verhältnismässig unerforschten frühen Entwicklung des Selbstkonzepts zwischen 6 und 8 Jahren befasst haben, wurde zusätzlich auch das Entwicklungsmodell des Selbstkonzepts von Harter herangezogen (1939, Neuauflage 2012). Diese zwei Modelle und weitere theoretische Grundlagen, von denen sich die drei Fragestellungen der Dissertation (vgl. Kapitel 1c-e) ableiten lassen, sollen im Folgenden kurz erläutert werden. Um Redundanz zu vermeiden, sei an einigen Stellen auf die drei Studien verwiesen, welche die konkreten empirischen Ergebnisse detaillierter erörtern. Die Bezeichnungen früh und jung beziehen sich im Folgenden stets auf die verhältnismässig frühe Entwicklung während der Schuleingangsphase von verhältnismässig jungen Kindern im Alter von ca. 6 bis 8 Jahren. 


\section{c) Validierung des Konstrukts Selbstkonzept}

Um die Konstruktvalidität des Selbstkonzepts zu prüfen, werden in der Selbstkonzeptforschung sogenannte Within- und Between-Netzwerk-Untersuchungen durchgeführt (Byrne, 1984). Within-Netzwerk-Untersuchungen nehmen sich der internen Binnenstruktur des Selbstkonzepts an. Das aktuell forschungsleitende Modell des Selbstkonzepts von Shavelson, Hubner, \& Stanton (1976) spezifiziert in erster Linie dieses Within-Netzwerk. Das Selbstkonzept wird dabei als multidimensionales, hierarchisch gegliedertes Gefüge beschrieben, an dessen Spitze das relativ stabile, generelle Selbstkonzept steht und in dessen Ausläufern die situations- und bereichsspezifischen Facetten des Selbstkonzepts zu finden sind. Letztere wiederum werden unterteilt in leistungsbezogene (z. B. akademisches Selbstkonzept) und nicht-leistungsbezogene Domänen (z. B. soziales, emotionales, physisches Selbstkonzept). Eine umfassende empirische Bestätigung dieses Modells liegt erst für Kinder ab der mittleren Grundschulzeit vor (z. B. Marsh, Byrne, \& Shavelson, 1988). Zum Within-Netzwerk des Selbstkonzepts von Kindern im Schuleingangsalter lässt sich festhalten, dass sich zwar bereits bereichsspezifische Selbstkonzeptfacetten messen liessen, dass über die Art und Anzahl der für den schulischen Bereich relevanten Facetten jedoch erst wenig Klarheit herrscht (z. B. Harter \& Pike, 1984; Mantzicopoulos, French, \& Maller, 2004; Marsh, Craven, \& Debus, 1991; Marsh et al., 2002).

Between-Netzwerk-Untersuchungen analysieren die Zusammenhänge zwischen generellen oder bereichsspezifischen Facetten des Selbstkonzepts und anderen Konstrukten wie beispielsweise Leistung und Interesse (z. B. Marsh, Trautwein, Lüdtke, Köller, \& Baumert, 2005), Leistungsmotivation (z. B. Spinath \& Spinath, 2005), Soziale Beziehungen (Measelle, 2005; Verschueren, Doumen, \& Buyse, 2012), Bindungsstile (Verschueren, Marcoen, \& Schoefs, 1996) und schulische Anpassungsfähigkeit (Verschueren, Buyck, \& Marcoen, 2001; Verschueren, Marcoen, \& Buyck, 1998). 
Einerseits stellen solche Between-Netzwerk-Untersuchungen nicht selten die Grundlage für umfassende Modelle dar, welche das Selbstkonzept als Komponente enthalten. Ein aktuelles Beispiel ist das Metacognitive and Affective Model of Self-Regulated Learning (MASRL-Modell) von Efklides (2011). Nach Efklides beinhaltet das akademische Selbstkonzept einen inhärenten metakognitiven (,,perceptions of ability are, in essence, metacognition about the self“, S.11), affektiven („,Thus, it seems that self-concept influences affect and affect influences self-concept", S.16) wie auch motivationalen Charakter („Selfconcept is also considered to have motivational power", S.11). Die Wechselwirkungen zwischen diesen drei Bereichen wiederum haben einen Einfluss auf das selbstregulierte Lernen eines Individuums. Erste empirische entwicklungspsychologische Daten von Kindern in der Schuleingangsphase zu diesem Modell stammen von Roebers, Cimeli, Röthlisberger und Neuenschwander (2012/iFirst).

Typische Between-Netzwerk-Studien widmen sich andererseits der Frage nach den Zusammenhängen zwischen dem mittlerweile sehr umfassend erforschten, akademischen Selbstkonzept und Leistungsmassen in Form von Leistungstests oder Noten (erste Metaanalyse von Hansford \& Hattie, 1982). Diese Korrelationen sind umso höher, je bereichsspezifischer Selbstkonzept- und Leistungsindikatoren definiert sind (Rost \& Sparfeldt, 2002). Die Zusammenhänge fallen vor und zu Beginn der Schullaufbahn gering aus und nehmen mit dem Alter substanziell zu (z. B. Bouffard, Marcoux, Vezeau, \& Bordeleau, 2003; Guay, Marsh, \& Boivin, 2003; Mantzicopoulos, 2006; Marsh et al., 2002). Hierbei spricht Helmke (1992) auch von einer zunehmenden Veridikalität, d. h. einer zunehmenden Realitätsangemessenheit der Selbstwahrnehmung eigener Fähigkeiten und Leistungen. Die Debatte, welche sich lange Zeit um die Wirkrichtung zwischen Selbstkonzept und Leistung entfacht hatte, ist mittlerweile durch die metaanalytisch ermittelten, empirischen Hinweise auf reziproke Wechselwirkungen zwischen den beiden Konstrukten etwas in den Hintergrund gerückt (reciprocal-effects-model, vgl. Chamorro-Premuzic, Harlaar, Greven, \& Plomin, 
2010; Marsh \& Martin, 2011; Valentine, DuBois, \& Cooper, 2004). Nachzuweisen sind sowohl eine Beeinflussung des nachfolgenden akademischen Selbstkonzepts durch die Leistung (skill-development-Ansatz, vgl. Calsyn \& Kenny, 1977) als auch umgekehrt, ein Einfluss vom vorangehenden akademischen Selbstkonzept auf die Leistung (selfenhancement-Ansatz, vgl. Calsyn \& Kenny, 1977). Dabei ist noch nicht abschliessend geklärt, ob spezifische Entwicklungsübergänge, wie beispielsweise der Schuleintritt, oder das Alter allgemein den Zusammenhang oder die Wirkrichtung beeinflussen, nicht zuletzt weil Studien mit jungen Kindern im Vorschul- und frühen Grundschulalter in den Metaanalysen unterrepräsentiert sind (vgl. Hansford \& Hattie, 1982; Helmke, 1992). Aufgrund der oben skizzierten Forschungsdefizite bei jungen Kindern stand im Sinne einer Konstruktvalidierung des frühen Selbstkonzepts als Erstes folgende übergreifende Fragestellung im Zentrum der Dissertation:

Fragestellung 1: Welche Eigenschaften weist entsprechend den Within- und BetweenNetzwerk-Ansätzen das Selbstkonzept von Kindern in der Schuleingangsphase auf?

\section{d) Selbstkonzeptgenese: Vergleichsprozesse}

Eine erste Vorstellung, welche Einflussfaktoren und Bedingungen bei der Selbstkonzeptgenese eine Rolle spielen, vermittelt uns wiederum das Selbstkonzeptmodell von Shavelson et al. (1976), welches einen ständigen Austauschprozess zwischen Innen- und Aussenwelt postuliert. Neben der Annahme, dass das eigene Selbstbild das Verhalten des Individuums beeinflusst, geht das Modell davon aus, dass das Selbstkonzept durch Informationen aus der Umwelt und deren Verarbeitung und Interpretation geformt wird. Wichtige Personen im Umfeld des Kindes, elterliche und pädagogische Bezugspersonen und Gleichaltrige, spielen dabei eine prominente Rolle (Harter, 1998). Im schulischen Kontext werden zwei wichtige Einflussfaktoren diskutiert: als externer Umweltfaktor das Rückmeldeverhalten der Eltern und Lehrpersonen (z. B. Bezugsnormorientierung nach 
Rheinberg, 2008) und auf Seite des Kindes verschiedene Vergleichsprozesse (Filipp, 1984; Hellmich, 2011). Es wird dabei zwischen externalen (d. h. sozialen), internalen (d. h. temporalen und dimensionalen) und kriterialen Vergleichsprozessen unterschieden (Möller \& Trautwein, 2009). Der soziale Vergleich (vgl. auch Festinger, 1954) gilt im schulischen Kontext als zentraler psychologischer Prozess der Selbstkonzeptgenese (Köller, 2004; Nicholls, 1978, 1984; Wagner, 1999). Schülerinnen und Schüler messen dabei ihre Leistungen im interindividuellen Vergleich zu den Leistungen anderer Mitschülerinnen und Mitschülern (Helmke, 1992). Abwärtsvergleiche wirken dabei selbstkonzeptförderlich, Aufwärtsvergleiche hingegen selbstkonzepthinderlich. Soziale Vergleichsprozesse können zum sogenannten Fischteicheffekt führen (engl. big-fish-little-pond-effect in Marsh, 1987), bei dem die individuelle Ausprägung der Selbsteinschätzung von dem durchschnittlichen Leistungsniveau der Bezugsgruppe abhängt. Beim temporalen Vergleich wird die aktuelle individuelle Leistung mit der Leistung in der Vergangenheit verglichen (Schöne, Dickhäuser, Spinath, \& Stiensmeier-Pelster, 2003), beim dimensionalen Vergleich die Leistung in einem Schulfach (z. B. Deutsch) mit der Leistung in einem anderen Schulfach (z. B. Mathematik, vgl. auch internal/external-frame-of-reference-Modell in Marsh, 1986). Ein kriterialer Vergleichsprozess findet dann statt, wenn ein Vergleich zwischen der aktuellen Leistung und einem objektiven Kriterium (z. B. Lehrplanvorgabe bzw. Vorgabe durch Lehrperson) angestellt wird.

Bei Kindern im Schuleingangsalter werden soziale und temporale Vergleichsinformationen als zentrale Quellen der Selbstkonzeptgenese vermutet (Dickhäuser \& Galfe, 2004; Helmke, 1992; Nicholls, 1984; Schöne et al., 2003). Erste Ergebnisse zu den Auswirkungen sozialer Vergleichsprozesse konnten bei dieser Altersgruppe in jüngster Zeit auch tatsächlich empirisch nachgewiesen werden (Abt Gürber, 2012; Gabriel, Kastens, Poloczek, Schoreit, \& Lipowsky, 2010). Da über die Selbstkonzeptgenese von Kindern in der Schuleingangsphase noch verhältnismässig wenig bekannt ist, wollten wir zudem der 
folgenden zweiten Fragestellung nachgehen:

Fragestellung 2: Welche Vergleichsprozesse stehen mit der frühen Selbstkonzeptgenese im Zusammenhang?

\section{e) Modell zur Entwicklung des Selbstkonzepts}

Da in dieser Dissertation die frühe Entwicklung des Selbstkonzepts von Kindern im Schuleingangsalter im Zentrum steht, das Modell von Shavelson et al. (1976) jedoch wenig entwicklungstheoretische Grundlagen beinhaltet, diente ein weiteres Modell zur Generierung von Fragestellungen und zur Interpretation der Ergebnisse. Es ist dies das Entwicklungsmodell von Harter (1939, Neuauflage 2012). Dieses umfasst eine detaillierte Beschreibung der Entwicklungsveränderungen im Selbstkonzept von der frühen, über die mittlere bis in die späte Kindheit. Gerade auch in der Schuleingangsphase spielen sich wichtige Veränderungen im Between- und Within-Netzwerk des Selbst ab (vgl. ,5 to 7 year shift“ in Harter, 1996). Was etwa die Entwicklung der Binnenstruktur des Selbstkonzepts betrifft, so beschreibt Harter zwei Richtungen, die auch von Marsh und Ayotte (2003) in der sogenannten „differential distinctiveness hypothesis“ erwähnt werden. Es findet im Entwicklungsverlauf einerseits eine zunehmende Ausdifferenzierung statt, indem sich beispielsweise einzelne Selbstkonzeptfacetten in den verschiedenen schulischen Fächern ausbilden. Andererseits vollzieht sich auf der Ebene der mentalen Repräsentationen auch eine Integration, nämlich dann, wenn Informationen von verschiedenen situationsspezifischen Merkmalen (z. B. einmalige Rückmeldung zu den Buchstabenkenntnissen durch die Kindergartenlehrkraft) zu einer höher-geordneten Generalisierung (Selbstkonzept: „Ich kann gut lesen und schreiben“) umgeformt werden.

Mit dem Bezug auf Neo-Piagetaner wie Fischer (1980) schafft Harter erstmals eine theoretische Verbindung zwischen der kognitiven Entwicklung und der Entwicklung des Selbstkonzepts (vgl. Between-Netzwerk). Indem sie das Selbstkonzept primär als kognitive 
Konstruktion betrachtet, widerspiegeln sich im Selbstkonzept die kognitiven Fähigkeiten und ihre spezifischen entwicklungsbedingten Limitationen. So bringt sie das typischerweise überoptimistische Selbstkonzept von Kindern im Schuleingangsalter erstens mit dem Allesoder-Nichts-Denken, d. h. der Denkweise in Form von Gegensätzen wie „gut“-,,schlecht“, in Verbindung (sog. representational mapping nach Fischer, 1980). Mit einer zunehmenden kognitiven Koordination dieser zuvor separiert vorliegenden Repräsentationen findet allmählich eine ausbalanciertere Selbstbeschreibung statt (sog. representational system nach Fischer, 1980). Diese führt dann allmählich zu einer Abnahme des überhöhten Selbstkonzepts, welche für die meisten Kinder wiederum mit einer Zunahme der Veridikalität des Selbstkonzepts verbunden ist (vgl. Marsh, Debus, et al., 2005). Ein weiterer Grund für die Überschätzung sieht Harter darin, dass Kinder in der Schuleingangsphase erst eingeschränkt über die kognitiven Fähigkeiten verfügen, welche nötig sind, um soziale Vergleichsinformationen konsequent für die Selbsteinschätzung nutzen zu können. Vielmehr sieht Harter (2012) die überhöhte Selbsteinschätzung als Hinweis, dass es temporale Vergleichsinformationen sind, welche in dieser Phase rapider Entwicklungsveränderungen salient sind und daher primär als Vergleichsrahmen dienen. Alles in allem wird die überhöhte Selbsteinschätzung in diesem jungen Alter als normativ betrachtet und ihr wird eine adaptive Funktion in der Ontogenese zugeschrieben (vgl. auch Bjorklund \& Bering, 2002; Bjorklund \& Green, 1992; Shin, Bjorklund, \& Beck, 2007). Da Theorie und erste empirische Studien Hinweise auf wichtige Entwicklungsveränderungen des Selbstkonzepts in der Schuleingangsphase geben, sollen Fragestellung 1 und 2 auch frühe Entwicklungsveränderungen einbeziehen:

\section{Ergänzung zu Fragestellung 1 und 2: Welche frühen Entwicklungsveränderungen} durchläuft das Selbstkonzept bezüglich seiner Ausprägung, seines Within- und BetweenNetzwerks und der Selbstkonzeptgenese? 
Auf ihrem Entwicklungsmodell aufbauend haben Harter und Pike (1984) mit der Pictoral Scale of Perceived Competence and Social Acceptance (PSPCSA) eines der ersten Messinstrumente des Selbstkonzepts für Kinder im Schuleingangsalter im englischsprachigen Raum entwickelt. Vom Inhalt und der Struktur her lehnt sich die in dieser Dissertation eingesetzte Selbstkonzeptskala an die PSPCSA an (für detailliertere Informationen siehe Kapitel 2c und Erläuterungen in Studie 1). Es stellte sich darum auch die methodische Frage nach der Eignung dieses eingesetzten Messinstruments:

Fragestellung 3: Inwiefern eignet sich die neu entwickelte Selbstkonzeptskala zur Messung des Selbstkonzepts bei Kindern in der Schuleingangsphase?

\section{Forschungsprojekt FIPS}

\section{a) Hintergrundinformationen zur Längsschnittstudie}

Zur Beantwortung der Forschungsfragen dienten Daten aus der Längsschnittstudie FIPS, welche von 2008 bis 2010 an der Universität Bern an der Abteilung Entwicklungspsychologie des Psychologischen Instituts durchgeführt wurde. Die zentrale Projektidee der Längsschnittstudie FIPS bestand darin, die Entwicklung von Kindern zwischen Vorschul- und frühem Grundschulalter zu verfolgen. Neben dem Selbstkonzept wurden mehrheitlich kognitive Merkmale wie Exekutive Funktionen, Nonverbale Intelligenz, mathematische und schriftsprachliche (Vorläufer)fertigkeiten, Lern- und Leistungsverhalten sowie sprachliche und motorische Merkmale untersucht. Die Längsschnittstudie umfasste drei Messzeitpunkte (MZP 1-3), die Messungen fanden jeweils am Ende des Schuljahres statt. In der Gesamtstichprobe waren $N=438$ Kinder. Die Kinder gehörten entweder dem traditionellen Schuleingangsmodell an, in welchem nach zwei Kindergartenjahren der Eintritt in die Grundschule erfolgt oder wurden in einem neuen Schuleingangsmodell beschult, welches die zwei Kindergartenjahre und die ersten beiden Grundschuljahre in der 
sogenannten „Basisstufe“ umfasst (vgl. Studie 3). Pro MZP wurden zudem Daten von zwei schulischen Jahrgängen erhoben (sog. ,,jüngere Kohorte“ und „ältere Kohorte“).

\section{b) Methodische Einbettung der drei Studien}

Die Dissertation fokussiert mehrheitlich auf die Daten des MZP 2 und des MZP 3. Studie 1 bezieht Daten des MZP 2 ein. Die Stichprobe, die dabei analysiert wurde, umfasste lediglich Kinder, die dem traditionellen Schuleingangsmodell angehörten. Die jüngere Kohorte befand sich zu diesem Zeitpunkt noch im Kindergarten, die ältere Kohorte war bereits in der 1. Klasse der Grundschule. In den Analysen der Studie 1 liess sich die Entwicklung dadurch querschnittlich betrachten. In der Studie 2 wiederum wurde die jüngere Kohorte aus Studie 1 vom Kindergarten (MZP 2) in die 1. Klasse (MZP 3) verfolgt. Damit bot sich die Gelegenheit, die zuvor querschnittlich betrachtete Entwicklung längsschnittlich zu untersuchen sowie dem Schuleintritt, als Ereignis, das zwischen MZP 2 und 3 stattfand, besondere Beachtung zu schenken. Studie 3 nahm sich einer etwas anderen Fragestellung an und untersuchte das Selbstkonzept als Bestandteil eines Netzwerks an Variablen, welche selbstreguliertes Lernen beeinflussen, in Abhängigkeit vom Schuleingangsmodell. Zu diesem Zweck wurde eine spezielle Teilstichprobe analysiert, welche Kinder beider Schuleingangsmodelle und beider Kohorten beinhaltet. Dabei wurden mehrheitlich Daten des MZP 3 analysiert. Die drei Studien unterscheiden sich auch hinsichtlich der Berücksichtigung der Selbstkonzeptfacetten: In Studie 1 stand die Frage nach unterschiedlichen Facetten des Selbstkonzepts im Vordergrund; darum waren drei Selbstkonzeptfacetten Gegenstand dieser Untersuchung. Studie 2 stellte die zwei in der bisherigen Forschung bereits erwähnten Facetten, das leistungsbezogene, vorakademische und das nicht-leistungsbezogene, soziale Selbstkonzept ins Zentrum; wir gingen davon aus, dass beide für den Schuleintritt von entscheidender Wichtigkeit sind. In Studie 3 wurde aus konzeptionellen Gründen lediglich das leistungsbezogene, (vor)akademische Selbstkonzept verfolgt, da im Modell von Efklides (2011) einzig diese Selbstkonzeptfacette als Bestandteil des selbstregulierten Lernens 
betrachtet wird. Tabelle $1 \mathrm{im}$ Anhang bietet einen Überblick über die methodischen Besonderheiten der drei Studien.

\section{c) Messinstrument zur Erfassung des Selbstkonzepts}

Da für Kinder in der Vorschul- und frühen Grundschulphase kein überzeugendes deutschsprachiges Messinstrument des Selbstkonzepts zur Verfügung stand, kam im Rahmen dieser Dissertation ein neues, bzw. für diese junge Altersgruppe adaptiertes Messinstrument zum Einsatz (siehe Erläuterungen in Studie 1). Zu Beginn des Forschungsprojekts wurde mit Hilfe einer Pilotstudie überprüft, ob diese Selbstkonzeptskala als Messinstrument eingesetzt werden kann. Dabei wurde das Selbstkonzept von $N=83$ Zweitklässlern sowohl anhand der deutschsprachigen Version der PSPCSA von Harter und Pike (1984, dt. Version für Grundschulkinder von Asendorpf \& van Aken, 1993), wie auch mittels der Selbstkonzeptskala erhoben. Die Unterskalen „,cognitive competence“ bzw. „social acceptance“ der PSPCSA und die Unterskalen „Vorakademisches Selbstkonzept“ (VaSK) bzw. „Soziales Selbstkonzept“ (SoSK) der Selbstkonzeptskala erfragen ähnliche, jedoch nicht exakt deckungsgleiche Themenbereiche. Die Subskalen korrelierten im leistungsbezogenen Bereich zu $r=.61, p<.001$ und im nicht-leistungsbezogenen, sozialen Bereich zu $r=.64$, $p<.001$. Diese Werte interpretierten wir als Hinweis auf eine akzeptable Übereinstimmungsvalidität der Selbstkonzeptskala (vgl. Fisseni, 1997).

Bei der einen Hälfte der Pilotierungsstichprobe $(n=37)$ wurde zudem das Leseverständnis (Salzburger Lesescreening nach Mayringer \& Wimmer, 2005) und die Schreibfertigkeiten (Hamburger Schreib-Probe nach May, 2002) und bei der anderen Hälfte der Stichprobe $(n=41)$ die mathematischen Fertigkeiten (Untertest Zahlenfolgen vom Heidelberger Rechentest nach Haffner, Baro, Parzer, \& Resch, 2005) erhoben. Die Korrelationen zwischen der „,ognitive competence“ (aus PCPCSA) bzw. dem VaSK (aus Selbstkonzeptskala) und den schulischen Fertigkeiten betrugen beim Leseverständnis $r=.43$, $p<.01$ bzw. $r=.36, p<.05$, bei den Schreibfertigkeiten $r=.33, p<.05$ bzw. $r=.38, p<.05$ 
und bei den mathematischen Fertigkeiten $r=.32, p<.05$ bzw. $r=.30, p<.10$. Diese

Ergebnisse interpretierten wir als weiteren Hinweis auf eine zufriedenstellende

kriteriumsbezogene Validität der Selbstkonzeptskala (vgl. Fisseni, 1997), die zumindest mit den Validitätsmassen des weltweit am häufigsten eingesetzten Messinstruments von Harter und Pike (1984) vergleichbar ist. Auch die Objektivität hinsichtlich der Durchführung wurde als gegeben angenommen. Zusammengenommen sprachen alle diese Werte insgesamt für den Einsatz der Selbstkonzeptskala bei dieser jungen Altersgruppe.

\section{Zusammenfassung der drei Studien}

Studie 1: Cimeli, P., Neuenschwander, R., Röthlisberger, M. \& Roebers, C. M. (im Druck). Das Selbstkonzept von Kindern in der Schuleingangsphase: Ausprägung und Struktur sowie Zusammenhänge mit frühen kognitiven Leistungsindikatoren. Zeitschrift für Entwicklungspsychologie und Pädagogische Psychologie.

In Studie 1 standen drei zentrale Aspekte des Selbstkonzepts (Ausprägung, Binnenstruktur und Zusammenhänge mit Aussenkriterien) und deren frühe Entwicklung im Vordergrund. Es ging dabei erstens um die Ausprägung bzw. die Höhe des Selbstkonzepts. Zweitens wurde untersucht, welche Binnenstruktur bzw. welche wichtigen, voneinander trennbaren Facetten das kindliche Selbstkonzept aufweist (Within-Netzwerk). Drittens ging es darum, zu überprüfen, welche Zusammenhänge zwischen dem Selbstkonzept und mathematischen bzw. schriftsprachlichen Vorläuferfertigkeiten und auch bereichsübergreifenden, für den Schulerfolg relevanten Fähigkeiten bestehen (BetweenNetzwerk).

Anhand der Analysen zur Binnenstruktur des Selbstkonzepts wurde über die erwarteten zwei Facetten des leistungsbezogenen, Vorakademischen Selbstkonzepts (VaSK) und des nicht-leistungsbezogenen, Sozialen Selbstkonzepts (SoSK) hinaus ein dritter, neuer Bereich des Selbst gefunden: das Spielbezogene Selbstkonzept (SpSK). Die kindliche 
Selbsteinschätzung im Puzzle zusammensetzen und Memory spielen, die unseres Wissens erstmals in einem Messinstrument erfragt wurden, grenzte sich dabei faktorenanalytisch und altersübergreifend klar von den beiden anderen vorgefundenen Facetten des Selbst ab.

Die Ergebnisse zeigten, dass das Selbstkonzept unabhängig vom erfragten Themenbereich und vom Alter hoch ausgeprägt war, was für ein optimistisches Selbstbild bzw. eine Selbstüberschätzung der Kinder in der Schuleingangsphase sprach und mit bisherigen Studienergebnissen in Einklang stand (z. B. Helmke, 1998; Mantzicopoulos, Patrick, \& Samapungavan, 2008). Hinsichtlich der Entwicklung in der Ausprägung zeigte sich beim Vergleich zwischen Kindergarten- und Schulkindern der querschnittliche Hinweis auf eine Zunahme im VaSK und Abnahmen im SoSK und SpSK. Es wurde vermutet, dass sich die Wahrnehmung des tatsächlichen, eigenen Leistungszuwachses in den Kulturtechniken Lesen, Schreiben und Rechnen zu Beginn der Grundschule in einem Anstieg des VaSK niederschlug. Dies wurde als Hinweis darauf interpretiert, dass insbesondere das leistungsbezogene Selbstkonzept in dieser Entwicklungsphase einem vorherrschenden Einfluss temporaler Vergleiche unterliegt (Dickhäuser \& Galfe, 2004; Harter, 2012).

Signifikante Zusammenhänge zwischen dem Selbstkonzept und mathematischen und schriftsprachlichen Vorläuferfertigkeiten einerseits und bereichsübergreifenden kognitiven Fähigkeiten andererseits traten wie erwartet lediglich beim VaSK auf und waren verhältnismässig niedrig. Ein Vergleich der Grösse dieser Zusammenhänge zwischen Kindergarten- und Schulkindern erbrachte keine Hinweise auf Entwicklungsveränderungen. Die eingesetzte Selbstkonzeptskala bot die Möglichkeit, mit dem Differenziertheitsmass ein neues Mass zur Quantifizierung der kindlichen Selbstbeschreibung einzuführen. Diese Variation der gewählten Rangpositionen über die gesamte Skala hinweg unterschied sich zwischen Kindergarten- und Schulkindern bedeutsam voneinander im Sinne einer Zunahme mit dem Alter. Zudem zeigten sich zwischen dem Differenziertheitsmass und den in Leistungstests erfassten bereichsübergreifenden und bereichsspezifischen 
(vor)schulischen Variablen bedeutsame Zusammenhänge. In Bezugnahme auf Harters (2012) postulierten Zusammenhänge zwischen der kognitiven Entwicklung und der Entwicklung des Selbst wurde das Differenziertheitsmass als potentielles, entwicklungssensitives Mass der Selbstkonzeptausdifferenzierung diskutiert.

Während damit in der Studie 1, die einmal mehr Daten eines stark überhöhten Selbstkonzepts lieferte, die normative Entwicklung im Vordergrund stand, drängte sich aus diesen Ergebnissen die Frage auf, welche Entwicklung Kinder aufweisen, welche kein so hoch ausgeprägtes Selbstkonzept haben. Studie 2 bot durch die Berücksichtigung der mittlerweile erhobenen Daten des MZP 3 die Chance, dieser Frage prospektiv im längsschnittlichen Entwicklungsverlauf nachzugehen.

Studie 2: Cimeli, P., Röthlisberger, M., Neuenschwander, R., \& Roebers, C. M. Stellt ein niedriges Selbstkonzept einen Risikofaktor für Anpassungsprobleme nach dem Schuleintritt dar? Zur Veröffentlichung eingereichtes Manuskript.

Ausgehend von der theoretischen Überlegung zur Adaptivität der kindlichen Selbstüberschätzung (z. B. Bjorklund \& Green, 1992) und der in Studie 1 gewonnenen empirischen Bestätigung, dass Kinder in der Schuleingangsphase häufig ein stark überhöhtes Selbstkonzept haben, wollten wir als nächstes der Frage nachgehen, welche Entwicklung Kinder mit einem untypisch niedrigen Selbstkonzept durchlaufen. Aus der self-enhancementPerspektive heraus wurde angenommen, dass ein niedriges Selbstkonzept einen Risikofaktor für die frühe schulische Anpassung darstellt, da es im Vergleich zu einem altersentsprechend ausgeprägten Selbstkonzept die Bewältigung der neuen, schulischen Anforderungen erschwert. In einem prospektiven Längsschnittdesign wurden die Kindergartenkinder $(N=103)$ aus Studie 1 nach dem Schuleintritt, ein Jahr später, im Bezug auf ihre leistungsmässige und soziale schulische Anpassung untersucht. Während sich die Kinder mit niedrigem Selbstkonzept (unterstes Terzil, $n=31$ ) von den Kindern mit altersentsprechend ausgeprägtem Selbstkonzept (Rest, $n=72$ ) im Kindergarten weder in soziodemographischen 
noch in kognitiven Merkmalen unterschieden, zeigten sie ein Jahr später mehrheitlich schlechtere Werte in der leistungsmässigen und sozialen schulischen Anpassung. Für eine praktische Bedeutsamkeit sprachen trotz relativ geringer statistischer Effektgrössen, was die Unterschiede zwischen den beiden Gruppen anging, zwei Argumente. Erstens schnitten die Kinder, die unmittelbar vor dem Schuleintritt über ein niedriges Selbstkonzept verfügten, in unterschiedlichen Bereichen, d. h. in Schulleistungen, im Leistungsverhalten und im Sozialverhalten schlechter ab, ein Muster, welches verschiedene Quellen (Eltern, Lehrpersonen, Leistungstests) bestätigten. Zweitens liessen eine mittlere Positionsstabilität des Selbstkonzepts und ein Vergleich der Klassifikationen zum ersten Erhebungszeitpunkt mit den Klassifikationen zum zweiten Erhebungszeitpunkt vermuten, dass für beinahe die Hälfte der Kindergartenkinder mit einem niedrigen Selbstkonzept eine erhöhte Wahrscheinlichkeit besteht, auch ein Jahr nach dem Schuleintritt ein niedriges Selbstkonzept zu haben. Da die Stabilität des Selbstkonzepts im Entwicklungsverlauf ansteigt (vgl. Helmke, 1991), ist es möglich, dass diese ursprünglich als praktisch nicht bedeutsam klassifizierten Nachteile im Entwicklungsverlauf zunehmen (vgl. Chapman et al., 2001). Als Forschungsausblick wurde auf die Dringlichkeit hingewiesen, diese Hypothese zu prüfen.

Während der Schwerpunkt in Studie 1 vorrangig auf den „,inneren“ Entwicklungs- und Reifungsprozessen des Selbstkonzepts bei jungen Kindern lag, wurde er in den Studien 2 und 3 allmählich auf „äussere“ Faktoren verlagert. In Studie 2 wurde der Schuleintritt explizit als äusserer Umweltfaktor und Zeitpunkt angenommen, an dem sich die Problematik eines niedrigen Selbstkonzepts auf besondere Art und Weise manifestiert. Studie 3 ging noch einen Schritt weiter und stellte die Frage ins Zentrum, ob die Art des Schuleingangsmodells als äusserer Umweltfaktor Einfluss auf die Selbstkonzeptgenese hat. 
Studie 3: Roebers, C. M., Röthlisberger, M., Neuenschwander, R., \& Cimeli, P. School progress, academic performance, and self-regulatory processes as a function of early elementary school context. [Schulischer Werdegang, schulische Leistungen und selbst-regulatorische Prozesse in Abhängigkeit des Schuleingangsmodells] Manuscript resubmitted for publication.

Die Kinder, welche in Studie 3 miteinander verglichen wurden, gehörten entweder dem traditionellen Schuleingangsmodell oder der Basisstufe an und waren bezüglich der Hintergrundvariablen Sozioökonomischer Status, Nonverbale Intelligenz, Informationsverarbeitungsgeschwindigkeit, Sprache, Alter und Geschlecht vergleichbar (sog. statistisches 1:1 Matching). Bisherige Studien, welche das Selbstkonzept in Abhängigkeit der Zugehörigkeit zu verschiedenen Schuleingangsmodellen untersucht haben, kamen zu widersprüchlichen Ergebnissen. Moser und Bayer (2010) und Vogt, Zumwald, Urech und Abt (2010), die mit der offiziellen Evaluation der Schulversuche beauftragt waren, fanden keine Unterschiede im Selbstkonzept der Kinder; ebensowenig Liebers (2008) sowie Beutel und Hinz (2008), welche die Evaluation deutscher Schuleingangsmodelle durchführten. Neuenschwander, Röthlisberger, Michel und Roebers (2011) fanden jedoch beim Vergleich der hier in Studie 3 bis zu MZP 3 verfolgten, gematchten Stichprobe (d. h. Kindergarten vs. Basisstufe) zu MZP 1, dass die älteren Kindergartenkinder (ältere Kohorte) über ein höheres vorakademisches und soziales Selbstkonzept verfügten als die Kinder in der Basisstufe; in der jüngeren Kohorte fanden sich diesbezüglich keine Unterschiede. Die Ergebnisse führten die Autorinnen darauf zurück, dass die älteren Kindergartenkinder als älteste ihrer Bezugsgruppe im sozialen Vergleich besser abschnitten als die gleichaltrigen Basisstufenkinder, deren Bezugsgruppe heterogener war (vgl. Fischteicheffekt, Marsh, 1987). Die Ergebnisse der im Rahmen der Dissertation durchgeführten Studie 3 ihrerseits sprechen dafür, dass der schulische Kontext zu MZP 3, zu dem sich die Kinder des traditionellen 
Schuleingangsmodells mehrheitlich in der 2. Grundschulklasse und die Kinder des neuen Schuleingangsmodells sich am Ende der Basisstufe befanden, keinen messbaren Einfluss (mehr) auf die Ausprägung des Selbstkonzepts hatte.

\section{Diskussion der Fragestellungen}

In folgendem Kapitel sollen die Ergebnisse der drei Dissertationsstudien anhand der drei Fragestellungen diskutiert werden. Beantwortung und Diskussion der Fragestellungen sind nach folgendem Prinzip aufgebaut: In einem ersten Schritt wird erläutert, inwiefern Studie 1-3 Ergebnisse und Erkenntnisse zu der Fragestellung liefern konnten. In einem zweiten Schritt werden Einschränkungen vorgenommen, mögliche praktische Implikationen erläutert und Forschungsausblicke vorgenommen. Zu erwähnen sei an dieser Stelle auch, dass zugunsten der Kohärenz und zur Vermeidung von Redundanz Erkenntnisse zu frühen Entwicklungsveränderungen nicht gesondert, sondern bereits integriert in Diskussion von Fragestellung 1 (bzgl. Within-und Between-Netzwerk) und 2 (bzgl. Selbstkonzeptgenese) diskutiert werden.

Fragestellung 1: Welche Eigenschaften weist entsprechend den Within- und BetweenNetzwerk-Ansätzen das Selbstkonzept von Kindern in der Schuleingangsphase auf?

In der Tradition des Within-Netzwerk-Ansatzes (vgl. Byrne, 1984) sollte zuerst die Frage nach der Anzahl der Facetten des Selbstkonzepts von Kindern in der Schuleingangsphase geklärt werden. Diesem Thema nahm sich vornehmlich Studie 1 an. Die Frage nach der Anzahl an Selbstkonzeptfacetten bei jungen Kindern mag etwas irreführend klingen und ist nicht absolut zu verstehen. Theoretisch ist hier nämlich eine unbegrenzte Anzahl einzigartiger Selbstdefinitionen denkbar (Rheinberg, 2008). Unser Vorhaben war vielmehr, uns hier gewissermassen auf mögliche für den (vor)schulischen Kontext bedeutsame Facetten zu beschränken. Unter Einsatz der auf die (vor)schulische Lern- und Erfahrungsumwelt von Kinder zwischen 6 und 8 Jahren ausgelegten Selbstkonzeptskala 
liessen sich so unabhängig vom Alter anhand einer explorativen Faktorenanalyse drei Facetten des Selbstkonzepts bestimmen: eine (vor)akademische, eine soziale und eine spielbezogene Facette. Da sich eine Differenzierung zwischen einem leistungsbezogenen (vorakademischen) und einem nicht-leistungsbezogenen (sozialen) Bereich bestätigen liess, lässt sich schlussfolgern, dass das Modell von Shavelson et al. (1976) auch für das Selbstkonzept von Kindern in der Schuleingangsphase eine gewisse Gültigkeit hat. Die Einordnung und auch die Bedeutung des spielbezogenen Selbstkonzept bleibt allerdings offen und müsste Untersuchungsgegenstand zukünftiger Studien insbesondere mit Vorschulkindern sein, deren Erlebniswelt noch stark vom Spiel geprägt ist (z. B. Pior, 1998; Rubin, Watson, \& Jambor, 1978). Dass in diesem jungen Alter weitere Selbstkonzeptfacetten für den (vor)schulischen Kontext von Belang sind, ist nicht auszuschliessen. In Anbetracht der prominenten Rolle von elterlichen und pädagogischen Bezugspersonen bei der Selbstkonzeptgenese (vgl. Harter, 1998) wäre möglicherweise eine Selbstkonzeptfacette, welche die Beziehung zwischen (Vor)schulkind und Lehrperson erfasst, ebenfalls von Bedeutung (analog zu "mütterlicher Akzeptanz" in Harter \& Pike, 1984; Leflot, Onghena, \& Colpin, 2010; Verschueren et al., 2012).

Einschränkend sei erwähnt, dass eine fächerspezifische Ausdifferenzierung des vorakademischen Selbstkonzepts in eine sprachliche und mathematische Selbstkonzeptfacette, wie sie Marsh et al. (1991) anhand einer konfirmatorischen Faktorenanalyse (CFA) gefunden haben, sich hier unter Umständen deshalb nicht zeigte, weil aufgrund des explorativen Charakters der Fragestellung und aufgrund unserer Stichprobengrösse eine explorative Faktorenanalyse durchgeführt wurde (vgl. auch Methodenargumentation in Marsh, Debus, et al., 2005). Möglicherweise liessen sich mit einer CFA durchaus auch Entwicklungsveränderungen in der Binnenstruktur bereits zwischen Kindergarten und Grundschule beobachten, die sich mit unseren Analysen nicht zeigten. 
Inwiefern die drei gefundenen Selbstkonzeptfacetten mit verschiedenen Variablen aus dem (vor)schulischen Kontext im Verbindung stehen bzw. dafür von Bedeutung sind, lässt sich ihren Zusammenhängen im Between-Netzwerk entnehmen (vgl. Byrne, 1984). Studie 1 nahm sich den Zusammenhängen zwischen verschiedenen Facetten des Selbstkonzepts und bereichsübergreifenden kognitiven Fähigkeiten bzw. bereichsspezifischen, mathematischen und sprachlichen Vorläuferfertigkeiten an. In Studie 2 wurde dem Between-Netzwerk-Ansatz durch die Betrachtung der Zusammenhänge zwischen Selbstkonzept und verschiedenen Indikatoren schulischer Anpassung Rechnung getragen. Da die Daten in Studie 2 zudem im Längsschnitt vorlagen, wurde im Sinne des self-enhancement-Ansatzes (nach Calsyn \& Kenny, 1977) die Auswirkung des Selbstkonzepts auf den weiteren Entwicklungsverlauf untersucht. Studie 3 lieferte ebenfalls Ergebnisse zum Between-Netzwerk-Ansatz, da neben den Zusammenhänge zwischen Selbstkonzept und Schulleistungen auch Verbindungen mit Prozessen selbstregulierten Lernens, nämlich Exekutiven Funktionen und Metakognitionsmassen untersucht wurden.

Was das Between-Netzwerk angeht, lässt sich erstens festhalten, dass sich zwischen vorakademischem Selbstkonzept und leistungsbezogenen Massen verhältnismässig konsistent signifikante Korrelationen ergaben, die allerdings niedrig (ca. $r \approx .20$ ) waren. Damit sind sie jedoch mit bisherigen Studienergebnissen von Kindern dieser Altersgruppe vergleichbar (z. B. Mantzicopoulos, 2006; Marsh et al., 2002). Das Zusammenhangsmuster zwischen akademischem Selbstkonzept und sprachlichen Schulleistungen $(r=.10$, n.s. im traditionellen Schulsystem und $r=.15$, n.s. in der Basisstufe) bzw. mathematischen Schulleistungen $(r=.15$, n.s. im traditionellen Schulsystem und $r=.25, p<.001$ in der Basisstufe $)$ in Studie 3 erscheint im Vergleich zu den Ergebnissen der Studie 1 und 2 vorerst inkonsistent und erstaunt insofern, als die Kinder in Studie 3 älter waren als in den beiden anderen Studien und mit dem Alter eine zunehmende Veridikalität zu erwarten wäre (vgl. Helmke, 1992; Marsh, Debus, et al., 2005). Hier liegt jedoch die Vermutung nahe, dass diese Zusammenhänge durch 
eine inzwischen fachspezifische Ausdifferenzierung des Selbstkonzepts verwischt wurden. Diese Hypothese wurde explorativ überprüft: Partialkorrelationen (Kontrollvariable Alter, $N=$ 227) zwischen dem sprachlichen Selbstkonzept mit den Komponenten „Buchstaben kennen“ und „Wörter lesen und schreiben“ bzw. dem mathematischen Selbstkonzept mit den Komponenten ,zählen“ und „rechnen“ und den sprachlichen Schulleistungen $(r=$. 29, $p<.001)$ bzw. den mathematischen Schulleistungen $(r=.16, p<.05)$ waren signifikant, die nicht-korrespondierenden Korrelationen hingegen waren - entsprechend der Regel der Bereichsspezifität (vgl. Rost \& Sparfeldt, 2002) - wie erwartet alle nicht signifikant. Zusammenfassend lässt sich schlussfolgern, dass Kinder in der Schuleingangsphase sich im vorakademischen Bereich nicht völlig losgelöst von den Leistungen, die sie in der Realität zeigen, einschätzen; dies gilt unabhängig vom Alter (ca. 6 - 8 Jahre) und Schulkontext (Kindergarten vs. Grundschule vs. Basisstufe). Dennoch scheinen die Zusammenhänge in diesem Alter nicht sonderlich robust zu sein (vgl. auch Hansford \& Hattie, 1982).

Zweitens waren zudem sowohl querschnittlich (Studie 1) wie auch längsschnittlich (Studie 2) bereichsspezifische (leistungsbezogene vs. nicht-leistungsbezogene) Zusammenhangsmuster beobachtbar. Aus der self-enhancement-Perspektive heraus betrachtet ist somit das vorakademische Selbstkonzept für die Aneignung (vor)schulischer Kompetenzen wie auch für die leistungsbezogene schulische Anpassung von Bedeutung. Das soziale Selbstkonzept wiederum scheint speziell für die soziale Anpassung eine wichtige Rolle zu spielen. Das spielbezogene Selbstkonzept hingegen zeigt keine Bezüge innerhalb des erhobenen Netzwerkes. Einschränkend sei anzumerken, dass in Studie 2 zwar von einem selfenhancement-Ansatz ausgegangen wurde, dass jedoch Effekte der umgekehrten Art (skilldevelopment-Ansatz) ebenso wirksam sein könnten, hier aber nicht explizit untersucht wurden (vgl. Aunola, Leskinen, Onatsu-Arvilommi, \& Nurmi, 2002; Bouffard et al., 2003; Chapman \& Tunmer, 1995; Zafiropoulou, Sotiriou, \& Mitsiouli, 2007). 
Was drittens das Zusammenwirken von Prozessen selbstregulierten Lernens angeht (vgl. MARSL-Modell nach Efklides, 2011), zeigten sich auf Korrelationsebene kaum signifikante Zusammenhänge zwischen dem vorakademischen Selbstkonzept, Exekutiven Funktionen und der Metakognition (vgl. Studie 3). Eine angemessenere statistische Methode wäre hier die Analyse mit Strukturgleichungsmodellen (vgl. Byrne, 2010), die geeignet sind, komplexe Zusammenhangsmodelle prüfen und zudem durch die Modellierung von latenten Variablen messfehlerbereinigte Daten beinhalten (vgl. Ergebnisse in Roebers et al., 2012/iFirst).

Eine Grösse, die sowohl zum Within- wie auch zum Between-Netzwerk des Selbstkonzept spannende Ergebnisse erbrachte, ist das Differenziertheitsmass. Das Mass widerspiegelt möglicherweise eine frühe Ausdifferenzierung des Selbstkonzepts, die sich stärker auf der Ebene einer veränderten Koordination der einzelnen Selbstbeschreibungen (vgl. representational system nach Fischer, 1980) abspielt als auf der Ebene einer veränderten Formation der unterschiedlichen Selbstkonzeptfacetten. Das Differenziertheitsmass stand zudem in einer verhältnismässig engen Verbindung mit den bereichsübergreifenden und bereichsspezifischen (vor)schulischen Variablen (vgl. Studie 1), folglich der kognitiven Entwicklung. Mit der Extraktion eines Differenziertheitsmasses lassen sich möglicherweise empirische Daten zu Harters (2012) postuliertem Zusammenhang zwischen der Selbstkonzeptentwicklung und der kognitiven Entwicklung gewinnen.

Insgesamt sprechen die vorliegenden Ergebnisse für eine Konstruktvalidierung des Selbstkonzepts von Kindern in der Schuleingangsphase. Dennoch bleiben auch einige Fragen dazu offen: Bezüglich des Within-Netzwerks wäre beispielsweise zu überprüfen, ob sich die Ergebnisse zum Differenziertheitsmass replizieren und erweitern lassen. Was das BetweenNetzwerk angeht, gilt es, zukünftig Zusammenhänge, die sich mit dem sozialen oder spielbezogenen Selbstkonzept ergeben, genauer zu untersuchen (vgl. Pior, 1998). Aufgrund der bisher gefundenen Hinweise auf frühe bereichsspezifische Zusammenhänge müsste man 
zudem überprüfen, ob sich der Risikofaktor „,niedriges generelles Selbstkonzept“ (vgl. Studie

2) auch auf bereichsspezifischer Ebene (niedriges vorakademisches bzw. soziales Selbstkonzept) finden lässt.

Fragestellung 2: Welche Vergleichsprozesse stehen mit der frühen Selbstkonzeptgenese im Zusammenhang?

An dieser Stelle soll vorweggenommen werden, dass keine der drei Studien eine Aussage zu den Vergleichsprozessen machen kann, die mit der frühen Selbstkonzeptgenese direkt im Zusammenhang stehen. Vielmehr lassen sich den Studien indirekte Hinweise auf zwei Vergleichsprozesse, namentlich die für den schulischen Kontext als zentral geltenden sozialen und temporalen Vergleiche (Dickhäuser \& Galfe, 2004; Helmke, 1992; Nicholls, 1984; Schöne et al., 2003), entnehmen, die möglicherweise in die frühe Selbstkonzeptgenese involviert sind. Dieser zweiten Fragestellung widmet sich Studie 3, welche Kinder unterschiedlicher Schuleingangsmodelle, unter anderem im Bezug auf ihr Selbstkonzept, miteinander vergleicht. Aufgrund der unterschiedlichen Bezugsgruppen werden Unterschiede in der Ausprägung des Selbstkonzepts erwartet. Bezugsgruppeneffekte werden als typische Auswirkungen sozialer Vergleichsprozesse angesehen (vgl. Marsh, 1987). Das Ausbleiben von Unterschieden in der Selbstkonzeptausprägung zwischen den Kindern der beiden Schuleingangsmodelle - wiederum unabhängig von der Altersgruppe - steht in Übereinstimmung mit anderen Studien (z. B. Beutel \& Hinz, 2008) und kann unterschiedlich interpretiert werden.

Einerseits könnte man annehmen, dass Kinder in diesem Alter tatsächlich, wie Harter (2012) annahm, für eine konsequente Nutzung sozialer Vergleiche kognitiv überfordert sind. Dass den Kindern soziale Vergleiche zumindest nicht gänzlich fremd waren, stellten sie jedoch dadurch unter Beweis, dass sie beim Einsatz der stark auf die Bezugsgruppe ausgerichteten Selbstkonzeptskala verhältnismässig valide Selbstkonzeptdaten lieferten. 
Andererseits ist aber auch möglich, dass Kinder auch aktiv mitbestimmen, wen sie als zur Bezugsgruppe zugehörig betrachten und darum Kinder des traditionellen Schulsystems und Kinder der Basisstufe - insbesondere gegen Ende der Basisstufe - eine vergleichbar homogene Bezugsgruppe für den sozialen Vergleich heranzogen.

Eine weitere Möglichkeit ist, dass soziale Vergleiche zwar wirkten, sie aber von temporalen Vergleichen „überschattet“" wurden; diese Erklärung wurde auch bei der Interpretation der Zunahme des vorakademischen Selbstkonzepts vom Kindergarten in die Grundschule in Studie 1 herangezogen. Diese Zunahme im vorakademischen Selbstkonzept liess sich im Übrigen auch längsschnittlich replizieren (vgl. Studie 2). Erst von der 1. in die 2. Klasse (bzw. von der 3. in die 4. Basisstufe) nahm das vorakademische Selbstkonzept nicht mehr zu, sondern geringfügig ab (vgl. Studie 3). Es lässt sich vermuten, dass die Entwicklungsfortschritte, welche Kinder in dieser Phase durchlaufen auch für die Kinder sehr salient sind und dass ein Fokus auf diese temporalen Vergleichsinformationen sogar ein funktionaler Mechanismus der Ontogenese darstellt (vgl. Bjorklund \& Bering, 2002).

Letztendlich lässt die Tatsache, dass sich auch keine Unterschiede in den Schulleistungen und Exekutiven Funktionen und kaum Unterschiede in den metakognitiven Fähigkeiten zwischen Kindern der beiden Schuleingangsmodellen zeigten, die Vermutung aufkommen, dass sich die Schuleingangsmodelle zu ähnlich waren, als dass sie auf die verschiedenen erhobenen Schülermerkmalen einen messbaren Einfluss haben (vgl. auch Moser \& Bayer, 2010; Vogt et al., 2010).

Da uns jedoch leider keine konkreten Unterrichtsvariablen zur Verfügung standen, bewegen wir uns mit diesen Überlegungen zu den Mechanismen, welche dazu geführt haben könnten, dass sich keine Unterschiede in der Ausprägung des Selbstkonzepts zwischen den beiden Schuleingangsmodelle zeigten, vorwiegend im Bereich der Spekulationen.

Als praktische Implikation für die Gestaltung der Schuleingangsphase liesse sich schlussfolgern, dass das Selbstkonzept gegenüber externen Faktoren, hier durch zwei 
unterschiedliche Schuleingangsmodelle repräsentiert, bereits früh über eine gewisse „Robustheit" verfügt, was konkret bedeutet, dass weder eine hohe, noch eine niedrige Altersund Leistungsheterogenität ein Risiko für die Entwicklung des Selbstkonzepts darstellt (vgl. auch Abt, 2012).

Im Rahmen eines Forschungsausblicks sei hier auch noch die Möglichkeit erwähnt, wie Studien direktere Hinweise auf Zusammenhänge zwischen Vergleichsprozessen und Selbstkonzeptgenese untersuchen könnten. Um beispielsweise den Einfluss von temporalen und sozialen Vergleichen auf die Selbstkonzeptgenese einander gegenüberzustellen, könnte zukünftig die Selbstkonzeptskala so angepasst werden, dass sie an beiden Polen nicht nur wie bisher den sozialen Vergleich herstellt (der/die Beste - der/die Schlechteste), sondern auch den temporalen Vergleich thematisiert (meine Leistung in x zu Beginn des Schuljahrs - meine Leistung in x zum jetzigen Zeitpunkt). Eine spezifische Erhebung, mit wem Kinder sich innerhalb einer potentiell zur Verfügung stehenden Bezugsgruppe vergleichen, wäre zudem ebenfalls informativ. Untersuchungen dieser Art würden weniger eine differenzielle als vielmehr eine prozessorientierte Forschungsperspektive (vgl. Krapp, 1997) einnehmen und insofern in ein Gebiet vorstossen, in dem gerade auch bei jüngeren Kindern grosser Forschungsbedarf herrscht.

Fragestellung 3: Inwiefern eignet sich die neu entwickelte Selbstkonzeptskala zur Messung des Selbstkonzepts bei Kindern in der Schuleingangsphase?

Grundsätzlich gilt, dass eine zuverlässige Erfassung des frühkindlichen Selbstkonzepts einige methodische Herausforderungen mit sich bringt; beispielsweise gilt es zu berücksichtigen, dass Kinder im Vor- und frühen Grundschulalter über ein eingeschränktes Konzentrations- und Sprachvermögen verfügen und mit einer Gruppenbefragung zum Selbstkonzept überfordert wären. Sie müssen deshalb individuell und mit Hilfe eines altersadäquaten Formats befragt werden (vgl. Asendorpf \& van Aken, 1993). Instrumente, 
welche die Testgütekriterien ausreichend erfüllen und das Selbstkonzept valide erfassen, sind ungeachtet dieser Schwierigkeiten die Grundlage für eine qualitativ hochstehende Forschung (Marsh, Debus, et al., 2005). Infolgedessen soll die hier eingesetzte Selbstkonzeptskala an dieser Stelle kritisch diskutiert werden. Einerseits wollen wir hier die Argumente, die für das Messinstrument sprechen, auflisten, andererseits auf mögliche Probleme, die mit dem Einsatz der Selbstkonzeptskala verbunden sind, eingehen. Wie bei den vorherigen Diskussionsteilen soll auch hier ein Ausblick auf zukünftige Forschungsvorhaben die Beantwortung der letzten Fragestellung abschliessen.

Was die Testgütekriterien betrifft liegen sowohl bezüglich Validität, Objektivität und Reliabilität der Selbstkonzeptskala akzeptable Werte vor, die sich durchaus mit den Gütekriterien herkömmlicher, englischsprachiger Messinstrumente für diesen Altersbereich wie der PSPCSA (Harter \& Pike, 1984) und dem Self Description Questionnaire for Preschoolers (Marsh et al., 1991) vergleichen lassen. Obschon man aufgrund der schiefen Verteilung des Selbstkonzepts und der niedrigen Zusammenhänge zwischen Selbstkonzept und Aussenkriterien auf eine mangelnde Validität des Messinstruments schliessen könnte, sprechen die bisherigen theoretischen und empirischen Befunde dafür, dies als normales Entwicklungsphänomen der Selbstüberschätzung denn als methodisches Problem zu interpretieren. Trotz der eingeschränkten Varianz, die man aufgrund des überhöhten Selbstkonzepts erwarten würde, vermochte das Messinstrument in seinem eher unüblichen Format gerade im oberen Bereich der Skala angemessen zu differenzieren. So sind dennoch individuelle Unterschiede messbar. Ein erster Hinweis, inwiefern diese Unterschiede auch prognostisch valide sind, lieferte Studie 2 mit ihrem prospektiven Längsschnittdesign.

Obschon das Format der Selbstkonzeptskala damit von Vorteil scheint und nicht zuletzt Grundlage zur Berechnung des Differenziertheitsmasses darstellt, beinhaltet es auch problematische Aspekte. So mag das soziale Vergleichsformat der Selbstkonzeptskala den Kindern zwar helfen sich möglichst adäquat einzuschätzen, gleichzeitig löst es 
höchstwahrscheinlich auch Bezugsgruppeneffekte aus (vgl. Marsh, 1987). Um diese in den Analysen mitzuberücksichtigen, wäre eine Bestimmung und Erhebung der Daten der gesamten Bezugsgruppe nötig, welche wiederum mehrebenenanalythisch ausgewertet werden müssten. Inwiefern allerdings das soziale Vergleichsformat tatsächlich andere Ergebnisse als übrige Vergleichsformate liefert, müsste anhand einer systematischen Gegenüberstellung verschiedener Vergleichsrahmen überprüft werden (vgl. Diskussion Fragestellung 2).

Was eine inhaltliche Überarbeitung angeht, wäre es wünschenswert, die Selbstkonzeptskala in allen drei Bereichen, dem vorakademischen, dem spielbezogenen und dem sozialen Bereich von der Itemanzahl her zu erweitern. Die Reliabilität der Unterskalen liesse sich damit vermutlich noch erhöhen (vgl. Asendorpf \& van Aken, 1993).

\section{Zusammenfassung und Schlussfazit}

In der vorliegenden Dissertation wurden die Konstruktvalidität des Selbstkonzepts, ausgewählte Faktoren der Selbstkonzeptgenese und diesbezügliche Entwicklungsveränderungen bei Kindern zwischen 6 und 8 Jahren untersucht. Es liessen sich erste Antworten auf Fragestellungen finden, die bisher vor allem bei Kindern ab der mittleren Grundschulzeit untersucht wurden. In den Ergebnissen wurden dabei einerseits Parallelen zur späteren Selbstkonzeptentwicklung sichtbar; beispielsweise in der Bedeutung des leistungsbezogenen Selbstkonzepts und seinen bereichsspezifischen Zusammenhänge innerhalb des Between-Netzwerks. Andererseits veranschaulichen die Ergebnisse beispielsweise anhand der hohen Selbstkonzeptausprägung und den Eigenschaften des frühen Within-Netzwerks auch die Besonderheiten der frühen Selbstkonzeptentwicklung in der Schuleingangsphase. Dank innovativen methodischen Aspekten wie einem neu eingesetzten Messinstrument oder der prospektiven Untersuchung von Kindern mit unterschiedlicher Selbstkonzeptausprägung konnte die Dissertation möglicherweise auch Anregungen für weitere Forschungsvorhaben liefern. Denn die Bedeutung des Selbstkonzepts für die 
(vor)schulische Erlebnis- und Lernwelt von Kindern in der Schuleingangsphase gilt es weiter zu erforschen. 


\section{Literaturverzeichnis}

Abt Gürber, N. (2012). Bereichsspezifische Selbstkonzepte bei Kindern in der

Schuleingangsstufe: Zusammenhänge mit Leistungen und Wohlbefinden in der Schule. Saarbrücken: Südwestdeutscher Verlag für Hochschulschriften.

Asendorpf, J. B. (2012). Persönlichkeitspsychologie. Berlin, Heidelberg: Springer Verlag.

Asendorpf, J. B., \& van Aken, M. A. G. (1993). Deutsche Versionen der Selbstkonzeptskalen von Harter. Zeitschrift für Entwicklungspsychologie und Pädagogische Psychologie, $25,64-86$.

Aunola, K., Leskinen, E., Onatsu-Arvilommi, T., \& Nurmi, J.-E. (2002). Three methods for studying developmental change: A case of reading skills and self-concept. British Journal of Educational Psychology, 72, 343-364.

Bandura, A. (1977). Self-efficacy: Toward a unifying theory of behavioral change. Psychological Review, 84(2), 191-215.

Beutel, S.-I., \& Hinz, R. (2008). Schulanfang im Wandel - Selbstkonzepte der Kinder als pädagogische Aufgabe. Berlin: LIT Verlag.

Bjorklund, D. F., \& Bering, J. M. (2002). The evolved child: Applying evolutionary developmental psychology to modern schooling. Learning and Individual Differences, $12,347-373$.

Bjorklund, D. F., \& Green, B. L. (1992). The adaptive nature of cognitive immaturity. American Psychologist, 47, 46-54.

Bouffard, T., Marcoux, M.-F., Vezeau, C., \& Bordeleau, L. (2003). Changes in selfperceptions of competence and intrinsic motivation among elementary school children. British Journal of Educational Psychology, 73, 171-186.

Byrne, B. M. (1984). The general/academic self-concept nomological network: A review of construct validation research. Review of Educational Research, 54, 427-456.

Byrne, B. M. (2010). Structural equation modeling with AMOS: Basic concepts, applications and programming. New York: Routledge.

Calsyn, R., \& Kenny, D. (1977). Self-concept of ability and perceived evaluations by others: Cause or effect of academic achievement? Journal of Educational Psychology, 69, $136-145$.

Chamorro-Premuzic, T., Harlaar, N., Greven, C. U., \& Plomin, R. (2010). More than just IQ: A longitudinal examination of self-perceived abilities as predictors of academic performance in a large sample of UK twins. Intelligence, 38, 385-392. 
Chapman, J. W., \& Tunmer, W. E. (1995). Development of young children`s reading selfconcepts: An examination of emerging subcomponents and their relationship with reading achievement. Journal of Educational Psychology, 87, 157-167.

Davis-Kean, P. E., \& Sandler, H. M. (2001). A meta-analysis of measures of self-esteem for young children: A framework for future measures. Child Development, 72, 887-906.

Dickhäuser, O., \& Galfe, E. (2004). Besser als..., schlechter als...: Leistungsbezogene Vergleichsprozesse in der Grundschule. Zeitschrift für Entwicklungspsychologie und Pädagogische Psychologie, 36, 1-9.

Efklides, A. (2011). Interactions of metacognition with motivation and affect in self-regulated learning: The MASRL model. Educational Psychologist, 46, 6-25.

Festinger, J. (1954). A theory of social comparison processes. Human Relations, 7, 117-140.

Filipp, S. H. (2006). Kommentar zum Themenschwerpunkt. Entwicklung von

Fähigkeitsselbstkonzepten. Zeitschrift für Entwicklungspsychologie und Pädagogische Psychologie, 20, 65-72.

Filipp, S. H. (Ed.). (1984). Selbstkonzept-Forschung: Probleme, Befunde, Perspektiven (Vol.

\section{2). Stuttgart: Klett-Cotta.}

Fischer. (1980). A theory of cognitive development: The control and construction of hierachies of skills. Psychological review, 87, 477-531.

Fisseni, H.-J. (1997). Lehrbuch der psychologischen Diagnostik. Göttingen/Toronto: Hogrefe.

Gabriel, K., Kastens, C., Poloczek, S., Schoreit, E., \& Lipowsky, F. (2010). Entwicklung des mathematischen Selbstkonzepts im Anfangsunterricht - Der Einfluss des Klassenkontextes. Zeitschrift für Grundschulforschung, 3, 65-82.

Guay, F., Marsh, H. W., \& Boivin, M. (2003). Academic self-concept and academic achievement: Developmental perspectives on their causal ordering. Journal of Educational Psychology, 95, 124-136.

Haffner, J., Baro, K., Parzer, P., \& Resch, F. (2005). Heidelberger Rechentest-HRT 1-4: Erfassung mathematischer Basiskompetenzen im Grundschulalter. Göttingen: Hogrefe.

Hansford, B. C., \& Hattie, J. A. (1982). The relationsship between self and achievement/performance measures. Review of Educational Research, 52, 123-142.

Harter, S. (1996). Developmental changes in self-understanding across the 5 to 7 shift. In A. J. Sameroff \& M. M. Haith (Eds.), The five to seven year shift: The age of reason and responsibility. London: Chicago Press. 
Harter, S. (1998). The development of self-representations. In W. Damon \& N. Eisenberg (Eds.), Handbook of Child Psychology: Social, Emotional, and Personality Development (pp. 553-617). New York: Wiley.

Harter, S. (2012). The construction of the self: A developmental perspective (Vol. 2). New York: Guilford Press.

Harter, S., \& Pike, R. (1984). The pictorial scale of perceived competence and social acceptance for young children. Child Development, 55, 1969-1982.

Hellmich, F. (Ed.). (2011). Selbstkonzepte im Grundschulalter: Modelle, empirische Ergebnisse, pädagogische Konsequenzen. Stuttgart: Kohlhammer.

Helmke, A. (1991). Entwicklung des Fähigkeitsselbstbildes vom Kindergarten bis zur dritten Klasse. In R. Pekrun \& H. Fend (Eds.), Schule und Persönlichkeitsentwicklung - ein Resümee der Längsschnittforschung (Vol. 11). Stuttgart: Ferdinand Enke Verlag.

Helmke, A. (1992). Selbstvertrauen und schulische Leistungen. Göttingen: Hogrefe.

Helmke, A. (1998). Vom Optimisten zum Realisten? Zur Entwicklung des Fähigkeitskonzeptes vom Kindergarten bis zur 6. Klassenstufe. In F. E. Weinert (Ed.), Entwicklung im Kindesalter. Weinheim: Beltz Psychologie Verlags Union.

James, W. (1890/1950). The Consciousness of Self. In J. William (Ed.), The principles of psychology. New York: Dover Publications.

Köller, O. (2004). Konsequenzen von Leistungsgruppierungen. Münster: Waxmann.

Krapp, A. (1997). Selbstkonzept und Leistung - Dynamik ihres Zusammenspiels: Literaturüberblick. In F. E. Weinert \& A. Helmke (Eds.), Entwicklung im Grundschulalter. Weinheim: Beltz.

Leflot, G., Onghena, P., \& Colpin, H. (2010). Teacher-child interactions: Relations with children`s self-concept in second grade. Infant and Child Development, 19, 385-405.

Liebers, K. (2008). Kinder in der flexiblen Einschulungsphase. Perspektiven für einen gelingenden Schulstart. Wiesbaden: VS Verlag für Sozialwissenschaften.

Mantzicopoulos, P. (2006). Younger children`s changing self-concept: Boys and girls from preschool through second grade. The Journal of Genetic Psychology, 167, 289-308.

Mantzicopoulos, P., French, B. F., \& Maller, S. J. (2004). Factoral structure of the pictoral scale of perceived competence and social acceptance with two pre-elementary samples. Child Development, 75, 1214-1228.

Mantzicopoulos, P., Patrick, H., \& Samapungavan, A. (2008). Young children`s motivational beliefs about learning science. Early Childhood Research Quarterly, 23, 378-394. 
Marsh, H. W. (1986). Verbal and math self-concepts: An internal/external frame of reference model. American Educational Research Journal, 23, 129-149.

Marsh, H. W. (1987). The big-fish-little-pond effect on academic self-concept. Journal of Educational Psychology, 79, 280-295.

Marsh, H. W., \& Ayotte, V. (2003). Do multiple dimensions of self-concept become more differentiated with age? The differential distinctiveness hypothesis. Journal of Educational Psychology, 95, 687-706.

Marsh, H. W., Byrne, B. M., \& Shavelson, R. J. (1988). A multifaceted academic selfconcept: Its hierachical structure and its relation to academic achievement. Journal of Educational Psychology, 80, 366-380.

Marsh, H. W., Craven, R. G., \& Debus, R. (1991). Self-concepts of young children 5 to 8 years of age: Measurement and multidimensional structure. Journal of Educational Psychology, 83, 377-392.

Marsh, H. W., Debus, R., \& Bornholt, L. (2005). Validating young children`s self-concept responses: Methodological ways and means to understand their responses. In M. D. Teti (Ed.), Handbook of research methods in developmental science. Oxford: Blackwell Publishing Ltd.

Marsh, H. W., Ellis, L. A., \& Craven, R. G. (2002). How do preschool children feel about themselves? Unraveling measurement and multidimensional self-concept structure. Developmental Psychology, 38, 376-393.

Marsh, H. W., \& Martin, A. J. (2011). Academic self-concept and academic achievement: Relations and causal ordering. British Journal of Educational Psychology, 81, 59-77.

Marsh, H. W., Trautwein, U., Lüdtke, O., Köller, O., \& Baumert, J. (2005). Academic selfconcept, interest, grades, and standardized test scores: Reciprocal effects models of causal ordering. Child Development, 76, 397-416.

May, P. (2002). Hamburger Schreib-Probe (HSP) 1-9 (6th ed.). Hamburg: vpm.

Mayringer, H., \& Wimmer, H. (2005). Salzburger Lese-Screening für die Klassenstufen 1-4 (SLS) (2nd ed.). Bern: Huber.

Measelle, J., R. (2005). Children`s self-perceptions as a link between familiy relationship quality and social adaptation to school. In P. Cowan, A., C. Cowan, P., J. Ablow, C., V. K. Johnson \& J. Measelle, R. (Eds.), The family context of parenting in children `s adaptation to elementary school. New Jersey: Lawrence Erlbaum Associates.

Möller, J., \& Trautwein, U. (2009). Selbstkonzept. In E. Wild \& J. Möller (Eds.), Pädagogische Psychologie. Heidelberg: Springer Medizin Verlag. 
Moschner, B. (2001). Selbstkonzept. In D. H. Rost (Ed.), Handwörterbuch Pädagogische Psychologie (2 ed.). Weinheim: Psychologie Verlags Union, Beltz.

Moser, U., \& Bayer, N. (2010). EDK-Ost 4bis8. Schlussbericht der summativen Evaluation. Lernfortschritte vom Eintritt in die Eingangsstufe bis zum Ende der 3. Klasse der Primarschule. Bern/Buchs: Schulverlag plus AG.

Neuenschwander, R., Röthlisberger, M., Michel, E., \& Roebers, C. M. (2011). Unterschiede in ausgewählten Bereichen der Schulfähigkeit: Ein Vergleich von Kindergarten und einem neuen Schuleingangsmodell in der Schweiz. Psychologie in Erziehung und Unterricht, 58, 30-40.

Nicholls, J. G. (1978). The development of the concepts of effort and ability, perception of academic attainment, and the understanding that difficult tasks require more ability. Child Development, 49, 800-814.

Nicholls, J. G. (1984). Achievement motivation: Conceptions of ability, subjective experience, task choice, and performance. Psychological Review, 91, 328-346.

Pior, R. (1998). Selbstkonzepte von Vorschulkindern- Empirische Untersuchungen zum Selbstkonzept sozialer Integration. Münster: Waxmann Verlag.

Rheinberg, F. (2008). Motivation (7 ed.). Stuttgart: Kohlhammer.

Roebers, C. M. (2007). Entwicklung des Selbstkonzeptes. In M. Hasselhorn \& W. Schneider (Eds.), Handbuch der Entwicklungspsychologie (pp. 381-391). Göttingen: Hogrefe.

Roebers, C. M., Cimeli, P., Röthlisberger, M., \& Neuenschwander, R. (2012/iFirst). Executive functioning, metacognition, and self-perceived competence in elementary school children: An explorative study on their interrelations and their role for school achievement. Metacognition \& Learning.

Rost, D. H., \& Sparfeldt, J. R. (2002). Facetten des schulischen Selbstkonzepts: Ein Verfahren zur Messung des differentiellen Selbstkonzepts schulischer Leistungen und Fähigkeiten (DISK-Gitter). Diagnostica, 48, 130-140.

Rubin, K., H., Watson, K. S., \& Jambor, T. W. (1978). Free-play behaviors in preschool and kindergarten children. Child Development, 49, 534-536.

Scheithauer, H., \& Petermann, F. (1999). Zur Wirkungsweise von Risiko- und Schutzfaktoren in der Entwicklung von Kindern und Jugendlichen. Kindheit und Entwicklung, 8, 314.

Schöne, C., Dickhäuser, O., Spinath, B., \& Stiensmeier-Pelster, J. (2003). Das Fähigkeitsselbstkonzept und seine Erfassung. In J. Stiensmeier-Pelster \& F. Rheinberg (Eds.), Diagnostik von Motivation und Selbstkonzept (pp. 3-14). Göttingen: Hogrefe. 
Schütz, A. (2003). Psychologie des Selbstwertgefühls: Von Selbstakzeptanz bis Arroganz (2 ed.). Stuttgart: Kohlhammer.

Shavelson, R. J., Hubner, J. J., \& Stanton, G. C. (1976). Self-concept: Validation of construct interpretation. Review of Educational Research, 46, 407-441.

Shin, H., Bjorklund, D., F., \& Beck, E. F. (2007). The adaptive nature of children`s overestimation in a strategic memory task. Cognitive Development, 22, 197-212.

Spinath, B., \& Spinath, F. M. (2005). Longitudinal analysis of the link between learning motivation and competence beliefs among elementary school children. Learning and Instructions, 15, 87-102.

Valentine, J., C., DuBois, D., L., \& Cooper, H. (2004). The relation between self-beliefs and academic achievement: A meta-analytic review. Educational Psychologist, 39, 111133.

Verschueren, K., Buyck, P., \& Marcoen, A. (2001). Self-representations and socioemotional competences in young children: A 3-year longitudinal study. Developmental Psychology, 37, 126-134.

Verschueren, K., Doumen, S., \& Buyse, E. (2012). Relationships with mother, teacher, and peers: Unique and joint effects on young children`s self-concept. Attachment \& Human Development, 14, 233-248.

Verschueren, K., Marcoen, A., \& Buyck, P. (1998). Five-year-old`s behaviorally presented self-esteem: Relations to self-perceptions and stability across a three-year period. The Journal of Genetic Psychology, 159, 273-279.

Verschueren, K., Marcoen, A., \& Schoefs, V. (1996). The internal working model of self, attachment, and competence in five-year-olds. Child Development, 67, 2493-2511.

Vogt, F., Zumwald, B., Urech, C., \& Abt, N. (2010). EDK-Ost 4bis8. Schlussbericht der formativen Evaluation. Grund-/Basisstufe: Umsetzung, Unterrichtsentwicklung und Akzeptanz bei Eltern und Lehrpersonen. Bern/Buchs: Schulverlag plus AG.

Wagner, J. W. L. (1999). Soziale Vergleiche und Selbsteinschätzungen: Theorien, Befunde und schulische Anwendungsmöglichkeiten. Münster: Waxmann Verlag.

Werner, E. E. (2007). Resilienz: Ein Überblick über internationale Längsschnittstudien. In G. Opp \& M. Fingerle (Eds.), Was Kinder stärkt - Erziehung zwischen Risiko und Resilienz. München: Ernst Reinhardt Verlag.

Zafiropoulou, M., Sotiriou, A., \& Mitsiouli, V. (2007). Relation of self-concept in kindergarten and first grade to school adjustment. Perceptual and Motor Skills, 104, $1313-1327$. 


\section{Anhang}

Tabelle 1

Übersicht über methodische Besonderheiten der durchgeführten drei Studien

\begin{tabular}{|c|c|c|c|}
\hline & Studie 1 & Studie 2 & Studie 3 \\
\hline $\begin{array}{l}\text { Fokussierte } \\
\text { Messzeitpunkte aus } \\
\text { FIPS }\end{array}$ & Messzeitpunkt 2 & Messzeitpunkt 2 und 3 & Messzeitpunkt 3 \\
\hline Stichprobe & $\begin{array}{l}N=297 \\
\text { Kinder des } \\
\text { traditionellen } \\
\text { Schuleingangsmodells }\end{array}$ & $\begin{array}{l}N=103 \\
\text { Kinder des } \\
\text { traditionellen } \\
\text { Schuleingangsmodells }\end{array}$ & $\begin{array}{l}N=230 \\
\text { Kinder des } \\
\text { traditionellen und der } \\
\text { Basisstufe }\end{array}$ \\
\hline Kohorte(n) & $\begin{array}{l}\text { - Jüngere Kohorte } \\
\text { (2. Kindergartenjahr) } \\
\text { - Ältere Kohorte } \\
\text { (1. Klasse) }\end{array}$ & $\begin{array}{l}\text { - Jüngere Kohorte } \\
\text { (2. Kindergartenjahr } \\
\text { bis 1. Klasse) }\end{array}$ & $\begin{array}{l}\text { - Jüngere } \\
\text { (1. Klasse bzw. } 3 . \\
\text { Basisstufenjahr) } \\
\text { - Ältere Kohorte } \\
\text { (2. Klasse bzw. } 4 . \\
\text { Basisstufenjahr) }\end{array}$ \\
\hline Entwicklung & querschnittlich & längsschnittlich & querschnittlich \\
\hline Selbstkonzept-facetten & $\begin{array}{l}\text { Vorakademisches } \\
\text { Selbstkonzept } \\
\text { - zählen } \\
\text { - rechnen } \\
\text { - Buchstaben kennen } \\
\text { - Wörter lesen und } \\
\text { schreiben } \\
\text { Soziales Selbstkonzept } \\
\text { - } \quad \text { Freunde haben } \\
\text { - } \quad \text { nebenan sitzen } \\
\text { - } \quad \text { Spielaufforder } \\
\quad \text { ung } \\
\text { - gemeinsam } \\
\quad \text { spielen/lernen } \\
\text { Spielbezogenes } \\
\text { Selbstkonzept } \\
\text { - Memory } \\
\text { - } \quad \text { Puzzle }\end{array}$ & $\begin{array}{l}\text { Vorakademisches } \\
\text { Selbstkonzept } \\
\text { - zählen } \\
\text { - rechnen } \\
\text { - Buchstaben kennen } \\
\text { - Wörter lesen und } \\
\text { schreiben } \\
\text { Soziales Selbstkonzept } \\
\text { - } \quad \text { Freunde haben } \\
\text { - } \quad \text { nebenan sitzen } \\
\text { - } \quad \text { Spielaufforder } \\
\quad \text { ung } \\
\text { - } \quad \text { gemeinsam } \\
\quad \text { spielen/lernen }\end{array}$ & $\begin{array}{l}\text { Vorakademisches } \\
\text { Selbstkonzept } \\
\text { - zählen } \\
\text { - rechnen } \\
\text { - Buchstaben kennen } \\
\text { - Wörter lesen und } \\
\text { schreiben }\end{array}$ \\
\hline
\end{tabular}




\section{Selbständigkeitserklärung}

Ich erkläre hiermit, dass ich diese Arbeit selbständig verfasst und keinen anderen als die angegebenen Quellen benutzt habe. Alle Stellen, die wörtlich oder sinngemäss aus Quellen entnommen wurden, habe ich als solche kenntlich gemacht. Mir ist bekannt, dass andernfalls der Senat gemäss Artikel 36, Absatz 1, Buchstabe o des Gesetztes vom 5. September 1996 über die Universität zum Entzug des aufgrund dieser Arbeit verliehenen Titels berechtigt ist. 
Studie 1: Cimeli, P., Neuenschwander, R., Röthlisberger, M. \& Roebers, C. M. (im Druck). Das Selbstkonzept von Kindern in der Schuleingangsphase: Ausprägung und Struktur sowie Zusammenhänge mit frühen kognitiven Leistungsindikatoren. Zeitschrift für Entwicklungspsychologie und Pädagogische Psychologie. 
Kolumnentitel: SELBSTKONZEPT IN DER SCHULEINGANGSPHASE

Das Selbstkonzept von Kindern in der Schuleingangsphase: Ausprägung und Struktur sowie Zusammenhänge mit frühen kognitiven Leistungsindikatoren

Self-concept of children at school entry: Mean level, structure, and relations to indicators of early cognitive achievement

Lic. phil. Patrizia Cimeli, Lic. phil. Regula Neuenschwander, Dr. phil. Marianne Röthlisberger, Prof. Dr. Claudia M. Roebers, Universität Bern

Die vorliegende Studie wurde finanziell unterstützt von der Jacobs Stiftung Zürich (Projekt: Transition to School: Developmental Pathways to Executive Control as a Function of Educational Experience). Wir danken den teilnehmenden Kindern, ihren Eltern und den Lehrpersonen und Schulleitungen für ihre Bereitschaft, an der Studie teilzunehmen. Ein großer Dank geht auch an unsere Projekt-MitarbeiterInnen für ihre tatkräftige Unterstützung.

Korrespondenzadresse:

Patrizia Cimeli

Universität Bern

Institut für Psychologie

Abteilung Entwicklungspsychologie

Muesmattstraße 45

CH-3000 Bern

Email: patrizia.cimeli@psy.unibe.ch 
Das Selbstkonzept von Kindern in der Schuleingangsphase: Ausprägung und Struktur sowie Zusammenhänge mit frühen kognitiven Leistungsindikatoren

Vor dem Hintergrund internationaler Vergleichsstudien ist in den letzten Jahren ein verstärktes Forschungsinteresse an der Schuleingangsphase zu verzeichnen, welche die Kindergarten- und die ersten Grundschuljahre umfasst. Dadurch sind die verschiedenen individuellen Schülermerkmale, die für einen erfolgreichen Übergang vom vorschulischen in das schulische Lernen von Bedeutung sind, in den Fokus der Forschung gerückt (OECD, 2006). Neben kognitiven und sozialen Aspekten scheinen auch affektiv-motivationale Merkmale für eine erfolgreiche schulische Anpassung wichtig zu sein (Daseking, Oldenhage \& Petermann, 2008; Harter, 1998; Wigfield \& Eccles, 2002). In der Literatur wird das Selbstkonzept, d. h. das kognitive Konstrukt der Selbstbeschreibung eigener Fähigkeiten und Merkmale, als ein zentrales affektiv-motivationales Merkmal aufgeführt (Harter, 1999; Meyer, 1984). Etliche Studien konnten die Bedeutung, welche diesem affektivmotivationalen Persönlichkeitsmerkmal speziell in schulischen Lern- und Leistungssituationen zukommt, vor allem bei älteren Schulkindern und Jugendlichen bestätigen (z. B. Guay, Marsh \& Boivin, 2003; Helmke, 1998; Spinath, Spinath, Harlaar \& Plomin, 2006). Dieser Vielfalt an Studien mit älteren Kindern steht ein Mangel an Untersuchungen des Selbstkonzepts von Kindern im Vorschul- und frühen Schulalter gegenüber, obschon in der Literatur vielfach postuliert wurde, dass dem Selbstkonzept schon in der Schuleingangsphase eine bedeutsame Rolle zukommt (z. B. Filipp, 2006; Harter, 1998; Marsh, Ellis \& Craven, 2002).

Die vorliegende Studie befasst sich deshalb mit der Ausprägung und der Struktur des Selbstkonzepts von Kindern in der Schuleingangsphase sowie den Zusammenhängen zwischen dem Selbstkonzept und den kognitiven Leistungsindikatoren. Mögliche Entwicklungsveränderungen hinsichtlich dieser drei Aspekte sollen durch systematische Vergleiche zwischen Kindern im Kindergarten und Kindern der ersten Grundschulklasse exploriert werden. Bereits vorliegende Ergebnisse für Kinder dieser Altersgruppen stammen vorwiegend aus dem englischsprachigen Raum. Entsprechend soll hier ein deutschsprachiges Messinstrument zur Erfassung des Selbstkonzepts entwickelt und eingesetzt werden. Dabei soll eine Erweiterung um einen Selbstkonzeptbereich vorgenommen werden, der in bisherigen Instrumenten unberücksichtigt blieb, nämlich den Bereich der spielerischen Aktivitäten. 
Erfassung des Selbstkonzepts von Kindern in der Schuleingangsphase

Ein Grund für den Mangel an Untersuchungen zum Selbstkonzept von Vorschul- und jungen Schulkindern mag darin liegen, dass die valide Erfassung des Selbstkonzepts aufgrund der eingeschränkten kognitiven und sprachlichen Ressourcen dieser Altersstufe relativ schwierig ist (Pior, 1998). Für den englischsprachigen Raum entwickelten Harter und Pike (1984) ein Messinstrument in Bildformat, die Pictoral Scale of Perceived Competence and Social Acceptance for Young Children (PSPCSA), welches leistungsbezogene Selbsteinschätzungen in kognitiven und körperlichen Fähigkeiten und nicht leistungsbezogene Selbsteinschätzungen bezüglich der Akzeptanz durch Gleichaltrige und der Akzeptanz durch die Mutter beinhaltet (PSPCSA, Version Vorschulkinder, Version 1./2. Klasse, deutsche Übersetzung für Kinder der 1./2. Klasse von Asendorpf \& van Aken, 1993). Dieses Messinstrument mit einem vierstufigen Antwortformat gilt als eines der am häufigsten eingesetzten Instrumente bei Kindern im Schuleingangsalter (Mantzicopoulos, 2006).

Eine etwas andere Messmethode, die ebenfalls bei jungen Kindern eingesetzt wird, geht auf Nicholls (1978) zurück. Sie beruht auf dem Prinzip, dass Kinder sich bezüglich verschiedener Eigenschaften und Fähigkeiten im Vergleich zu ihrer Klasse bzw. ihrer Kindergartengruppe einschätzen (sogenanntes soziales Vergleichsformat). Im Einzeltest wird das Kind aufgefordert, seine Position in einer Reihe von Kindern, die als Figuren oder bildlich dargestellt sind, zu kennzeichnen. Diese Messmethode kommt vor allem im deutschsprachigen Raum zur Anwendung, so z. B. in der Münchner LOGIK-Studie bei Helmke (1998), bei Roebers und Schneider (1999), bei Röthlisberger und Michel (2009), bei Neuenschwander, Röthlisberger, Michel und Roebers (2011) und Niklas und Schneider (in Druck). Vereinzelt wird sie auch außerhalb des deutschsprachigen Raums angewendet, so z. B. bei Nurmi und Aunola (2005) sowie Stipek und Daniels (1988). Die Visualisierung der sozialen Bezugsgruppe in dieser Messmethode soll jungen Kindern ein besseres Verständnis der Aufgabe sowie eine genauere Selbsteinschätzung ermöglichen. Soziale Vergleichsinformationen gelten zudem - speziell im Kontext Schule - neben internaltemporalen Vergleichsinformationen als die zentralen Quellen für die Selbstkonzeptgenese bei Kindern im Schuleingangsalter (Dickhäuser \& Galfe, 2004; Helmke, 1992; Köller, 2004; Nicholls, 1978, 1984; Wagner, 1999).

Die in der vorliegenden Untersuchung eingesetzte Selbstkonzeptskala lehnt sich inhaltlich an die zwei Skalen der kognitiven Fähigkeiten und der Akzeptanz durch 
Gleichaltrige der PSPCSA von Harter und Pike (1984) an, bedient sich von der Form her jedoch des sozialen Vergleichsformats. Um inhaltlich zudem Aspekte der spielerischen Lern- und Erfahrungswelt von Kindern im Vorschul- und frühen Schulalter erfassen zu können, wurde zusätzlich die Selbsteinschätzung in Spielen erfragt, welche neben kreativen auch speziell kognitive Anforderungen beinhalten (für eine Übersicht siehe Pior, 1998).

Bisherige Forschungsergebnisse bezüglich der drei Fragestellungen dieser Studie, die dank der Fortschritte in der Entwicklung von kindgerechten Messinstrumenten möglich wurden, sollen nun vorgestellt werden.

Ausprägung des Selbstkonzepts

Das Selbstkonzept von Kindern in der Schuleingangsphase ist typischerweise deutlich überhöht (French \& Mantzicopoulos, 2007; Harter, 1998; Helmke, 1998; Nicholls, 1978; Shin, Bjorklund \& Beck, 2007). Diese robuste Selbstüberschätzung zeigt sich unabhängig vom eingesetzten Messinstrument in einer schiefen Verteilung und einer Höhe, welche deutlich über dem zu erwartenden Mittelwert der jeweiligen Skala liegt (z. B. Asendorpf \& van Aken, 1993; Helmke, 1998; Mantzicopoulos, 2006). Im Entwicklungsverlauf sinkt die Ausprägung des Selbstkonzepts allmählich ab (Eccles \& Wigfield, 2002; Jacobs, Lanza, Osgood, Eccles \& Wigfield, 2002; Marsh \& Ayotte, 2003; Roebers \& Schneider, 1999), seine Höhe entspricht allerdings selbst bei Erwachsenen noch einer leichten Überschätzung der eigenen Person (positive illusory bias, z. B. Robins \& Beer, 2001). Das Absinken des Selbstkonzepts wird mit Fortschritten in der kognitiven Entwicklung (z. B. Sprache, Metakognition, Perspektivenübernahme) in Verbindung gebracht (Harter, 1998). Auch Faktoren im sozialen Umfeld der Kinder wie schulische Leistungsrückmeldungen und familiäres Feedback führen in der Ontogenese zu einem angemesseneren Selbstbild (Beutel \& Hinz, 2008; Filipp, 1984; Roebers, 2007). Einige Untersuchungen sind der Frage nachgegangen, von welchem Alter an sich eine Abnahme des Selbstkonzepts erstmals nachweisen lässt. Zwei Studien finden bereits ein vom Kindergartenalter an absinkendes Selbstkonzept (Mantzicopoulos, 2006; Marsh, Craven \& Debus, 1991). Insbesondere für das leistungsbezogene Selbstkonzept werden auch immer wieder Zunahmen in der Ausprägung dokumentiert, welche teilweise bis ins zweite Schuljahr andauern (Aunola, Leskinen, Onatsu-Arvilommi \& Nurmi, 2002; Beutel \& Hinz, 2008; Helmke, 1998; Mantzicopoulos, 2006; Renner, 2008). Die bisherigen empirischen Studien zu frühen Entwicklungsveränderungen in der Ausprägung des Selbstkonzepts liefern 
demnach inkonsistente Ergebnisse und zeigen die Notwendigkeit weiterer Forschungsarbeiten auf.

Struktur des Selbstkonzepts

Ein zentraler Bestandteil des hierarchischen Selbstkonzeptmodells von Shavelson, Hubner und Stanton (1976) ist die Annahme, dass sich im Entwicklungsverlauf eine zunehmende Anzahl von Selbstkonzeptbereichen ausbildet. Diese sogenannte Ausdifferenzierung konnte vor allem für ältere Kinder und Jugendliche bestätigt werden (z. B. Marsh \& Ayotte, 2003). Für Kinder in der Schuleingangsphase jedoch liegen bisher nur wenige und widersprüchliche Ergebnisse zur Anzahl der faktorenanalytisch trennbaren Selbstkonzeptbereiche vor (Marsh et al., 1991). Studien, welche die PSPCSA einsetzten, fanden Hinweise auf eine Selbstkonzeptstruktur, die lediglich aus einem leistungsbezogenen und einem nicht leistungsbezogenen Bereich besteht (Harter \& Pike, 1984; Mantzicopoulos, French \& Maller, 2004). Marsh et al. (1991) hingegen konnten die acht Skalen des Self Description Questionnaire for Preschoolers (SDQ-P von Marsh et al., 2002) bereits bei Kindern zwischen 5 und 8 Jahren empirisch bestätigen (allgemeines Selbstkonzept, Selbstkonzept für Schule, Mathematik, Lesen, körperliche Fähigkeiten, körperliche Erscheinung, Selbsteinschätzung der Beziehung zu den Gleichaltrigen und zu den Eltern). Neben einer zunehmenden Anzahl an empirisch trennbaren Selbstkonzeptbereichen im Entwicklungsverlauf wird eine Abnahme der Korrelationen zwischen den Bereichen als zusätzlicher Hinweis für eine ansteigende Ausdifferenzierung betrachtet (Marsh \& Hocevar, 1985).

\section{Zusammenhänge zwischen Selbstkonzept und kognitiven Leistungsindikatoren}

Für ältere Schulkinder und Jugendliche gibt es zahlreiche Studien, die bedeutsame mittlere bis hohe - positive Zusammenhänge zwischen leistungsbezogenem Selbstkonzept und Außenkriterien, d. h. objektiven Leistungsmaßen oder Schulnoten, dokumentieren (Guay et al., 2003; Helmke, 1998). Diese Zusammenhänge nehmen im Entwicklungsverlauf zu (z. B. Bouffard, Marcoux, Vezeau \& Bordeleau, 2003). Für Kinder in der Schuleingangsphase fallen die Zusammenhänge geringer aus. Eine Metaanalyse von Hansford und Hattie (1982) fand für Kindergartenkinder eine durchschnittliche Korrelation von $r=.12$ und für Grundschulkinder eine Korrelation von $r=.20$ zwischen dem leistungsbezogenen Selbstkonzept und der Performanz. Aktuelle Studien berichten über geringe, aber signifikante Korrelationen von $r=.10$ bis $r=.40$ (Buff, Nakamura, Hollenweger \& Achermann, 2005; Hughes, Dyer, Luo \& Kwok, 2009; Mantzicopoulos, 
2006; Marsh et al., 2002; Martschinke \& Kammermeyer, 2003). Dabei erweist sich der Zusammenhang zwischen Selbstkonzept und Leistung schon bei jüngeren Kindern als bereichsspezifisch (sogenannte Regel der Bereichsspezifität): Je ähnlicher Selbstkonzeptund Leistungsindikatoren definiert sind, umso größer ist ihr statistischer Zusammenhang (Rost \& Sparfeldt, 2002). Die Untersuchung des Zusammenhangs zwischen Selbstkonzept und Leistung in der Schuleingangsphase bringt - speziell bei Kindergartenkindern - gewisse methodische Herausforderungen mit sich: Anstelle von schulischen Leistungsindikatoren (Schulleistungstest, Noten) müssen andere Außenkriterien gewählt werden, z. B. sogenannte schulische Vorläuferfertigkeiten. Für die Mathematik werden unter anderem frühe Zählfertigkeiten (Krajewski \& Schneider, 2006), für den Schriftspracherwerb neben der phonologischen Bewusstheit (Näslund \& Schneider, 1996) auch die lexikalische Zugriffsgeschwindigkeit (Anthony, Williams, McDonald \& Francis, 2007) als wichtige Vorläuferfertigkeiten diskutiert. Bei Kindern in der Schuleingangsphase erscheint es zudem sinnvoll, als Außenkriterien zusätzlich bereichsübergreifende kognitive Fähigkeiten einzubeziehen, welche bedeutsame Prädiktoren für erfolgreiche schulische Anpassung und Schulfähigkeit sind. Dazu gehören die Fluide Intelligenz und das Arbeitsgedächtnis (z. B. Blair \& Razza, 2007; Krajewski \& Schneider, 2009), aber auch das Konstrukt der Exekutiven Funktionen, das als kognitive Komponente selbstregulatorischer Fähigkeiten definiert wird (z. B. Best, Miller \& Jones, 2009).

Hinsichtlich der Ausprägung des Selbstkonzepts werden in der vorliegenden Untersuchung grundsätzlich stark überhöhte Selbstkonzeptwerte erwartet. Wir nehmen jedoch an, dass sich Kindergarten- und Schulkinder in der Höhe des Selbstkonzepts unterscheiden werden. Aufgrund widersprüchlicher bisheriger Befunde verzichten wir hier auf eine spezifische Hypothese, was die Richtung dieser Unterschiede betrifft. Im Hinblick auf die Struktur des Selbstkonzepts soll überprüft werden, welche Bereiche sich bei Kindern in der Schuleingangsphase nachweisen lassen, wenn mit dem neu eingesetzten Messinstrument speziell auch Themen berücksichtigt werden, die der spielerischen Erfahrungswelt junger Kinder entstammen. Aufgrund bisheriger wissenschaftlicher Befunde erwarten wir, dass Kinder in der Schuleingangsphase mindestens zwischen einem leistungsbezogenen und einem nicht leistungsbezogenen Selbstkonzeptbereich unterscheiden werden. Es wird auch erwartet, dass Schulkinder allgemein eine stärkere Ausdifferenzierung des Selbstkonzepts aufweisen werden als Kindergartenkinder. Als Letztes stellt sich die Frage, ob und welche Zusammenhänge sich zwischen dem Selbstkonzept und verschiedenen 
kognitiven Leistungsindikatoren bei Kindern im Schuleingangsalter nachweisen lassen. Gemäß der Regel der Bereichsspezifität erwarten wir die - relativ gesehen - größten Zusammenhänge zwischen dem leistungsbezogenen Selbstkonzept und den schulischen Vorläuferfertigkeiten. Diese Zusammenhänge sollten bei Schulkindern deutlicher erkennbar sein als bei Kindergartenkindern.

\section{Methode}

\section{Stichprobe}

Die Stichprobe bestand aus $N=297$ Kindern (54 \% Knaben). Davon besuchten 110 Kinder im Alter von $\mathrm{M}=79.8$ Monaten $(S D=3.7)$ den Kindergarten (Kindergartenkinder) und 187 Kinder im Alter von $\mathrm{M}=90.6$ Monaten $(S D=4.1)$ die 1. Klasse der Grundschule (Schulkinder). Die zwei Altersgruppen stammten aus insgesamt 79 Kindergärten bzw. Schulklassen aus ländlichen und städtischen Regionen verschiedener deutschsprachiger Kantone der Schweiz. Pro Kindergarten bzw. Schulklasse konnten ca. 4 Kinder $(\mathrm{M}=3.8, S D=2.9)$ getestet werden, dies bei einem Minimum von einem Kind und einem Maximum von 14 Kindern pro Gruppe. Die Stichprobe umfasste 79 \% (schweizer-)deutschsprachige, $16 \%$ fremdsprachige und $5 \%$ zweisprachige Kinder. Versuchsablauf

Im Rahmen des Forschungsprojekts Übergang in die Schule führten instruierte VersuchsleiterInnen (VL) jeweils vormittags während 30 Minuten im Einzelsetting Testungen durch. Zur Untersuchung zugelassen waren alle Kinder, deren Eltern ein aktives Einverständnis zur Teilnahme an der Studie gegeben hatten. Die Testungen fanden in einem ruhigen Raum innerdhalb der (vor)schulischen Einrichtung im letzten Schuljahresquartal statt. Als Dankeschön erhielt jedes Kind ein kleines Geschenk.

\section{Messinstrumente}

Zur Erhebung des Selbstkonzepts diente die Selbstkonzeptskala, welche den Kindern als Papier-Bleistift-Individualtest vorgelegt wurde, wie er in Abbildung 1 gezeigt wird. Die Skala war bereits im Rahmen einer unveröffentlichten Pilotstudie zusammen mit dem PSPCSA bei $N=83$ Zweitklässlern eingesetzt worden. Die Korrelationen zwischen den entsprechenden Skalen der PSPCSA und der Selbstkonzeptskala betrugen zwischen $r=.61$ und $r=.64, p<.01$. Zudem berichteten Cimeli, Neuenschwander, Röthlisberger und Roebers (2010) bei einer Stichprobe von $N=207$ Erstklässlern Korrelationen in der Höhe von $r=.32, p<.01$, zwischen dem leistungsbezogenen Selbstkonzept und den Lese- und Mathematikleistungen. 


\section{Abbildung 1 - hier einfügen}

Die Selbsteinschätzung der Kinder wurde mithilfe einer vertikalen Rangordnung von je 25 geschlechtsneutralen Figuren erfasst. Zur Beispielaufgabe "Körpergröße" wurde folgende Instruktion abgegeben:

Schau mal, hier ist eine Reihe von Kindern. Stell dir vor, dies sind die Kinder deiner Klasse (bzw. Kindergartengruppe). Hier haben sich die Kinder deiner Klasse (bzw. Kindergartengruppe) der Größe nach aufgestellt. Dieses Kind hier (VL zeigt auf die oberste Figur) ist das größte Kind der Klasse, dieses Kind hier (VL zeigt auf die unterste Figur) ist das kleinste Kind und dieses Kind hier (VL zeigt auf die Figur auf der Mittellinie) befindet sich genau in der Mitte. Welches von diesen Kindern bist $\mathrm{du}$ ?

Diese Instruktion wurde für die sechs Themenbereiche „Buchstaben kennen“, „Wörter lesen und schreiben“, „Zählen“, „Rechnen“, „Puzzle zusammensetzen“ und „Memory spielen“ wiederholt. Anstelle von ,der Größte/der Kleinste“ wurde die Formulierung ,die/der Beste im ...“ und ,die/der Schlechteste im ...“ wiederholt. Bei den vier Themenbereichen, die keine Fähigkeiten abbildeten (viele Kinder wollen vs. niemand will neben ihr/ihm sitzen, oft vs. nie zum Spielen aufgefordert werden, viele vs. keine Freunde haben, oft mit anderen Kindern zusammen vs. oft alleine spielen und lernen) wurde die Instruktion wie folgt angepasst:

Dieses Kind hier (VL zeigt auf die oberste Figur) hat viele Freunde und dieses Kind hier (VL zeigt auf die unterste Figur) hat keine Freunde. Dieses Kind hier (VL zeigt auf die Mittellinie) liegt genau dazwischen. Welches von diesen Kindern bist du? Als abhängige Variable der Selbstkonzepthöhe diente die markierte Position auf der vertikalen Reihe (Minimum 1 = unterste Position bis Maximum $25=$ oberste Position). Um die Hypothese der zunehmenden Ausdifferenzierung des Selbstkonzepts zu prüfen, wurde eine weitere abhängige Variable aus den 10 Themenbereichen extrahiert: Das Differenziertheitsmaß (DM). Dabei handelte es sich um die Anzahl der sich voneinander unterscheidenden Positionen, die ein Kind über alle Themenbereiche hinweg gewählt hatte. So erhielt beispielsweise ein Kind, das sich in allen 10 Themenbereichen mit der mittleren Figur identifiziert hatte, ein DM von 1. Ein Kind, welches sechs verschiedene Positionen gewählt hatte (z. B. zweimal bei 1, zweimal bei 13, je einmal bei 15, 23 und 24 und fünfmal bei 25), bekam unabhängig von den konkret gewählten Werten in der Selbstkonzepthöhe ein DM von 6. Nach dem gleichen Prinzip wurde jeweils ein DM für die vier vorakademischen 
Themenbereiche $\left(\mathrm{DM}_{\text {vorakademisch }}\right)$ und die vier sozialen Themenbereiche $\left(\mathrm{DM}_{\text {sozial }}\right)$ berechnet (siehe auch die Ergebnisse zur Faktorenstruktur der Selbstkonzeptskala).

Um berechnen zu können, welche Zusammenhänge zwischen dem Selbstkonzept und den kognitiven Leistungsindikatoren von Kindergarten- und Schulkindern existieren, wurden einerseits bereichsübergreifende kognitive Kompetenzen (Fluide Intelligenz, phonologische Schleife des Arbeitsgedächtnisses, Exekutive Funktionen) und andererseits schulische Vorläuferfertigkeiten im mathematischen und schriftsprachlichen Bereich erhoben.

Die Fluide Intelligenz wurde anhand des Intelligenztests Test of Nonverbal Intelligence (TONI-3) von Brown, Sherbenou und Johnson (1997) gemäß Handanweisung erhoben. Als abhängige Variable diente die Anzahl korrekter Antworten.

Mit der Aufgabe Zahlen nachsprechen wurde die phonologische Schleife des Arbeitsgedächtnisses erfasst (Krajewski \& Schneider, 2009). Die Kinder mussten dabei eine vorgesprochene Zahlenfolge in der gleichen Reihenfolge mündlich wiedergeben. Pro Spanne wurden drei Zahlensequenzen vorgegeben. Die Aufgabe wurde nur dann fortgesetzt, wenn mindestens zwei Sequenzen korrekt waren. Als abhängige Variable diente die Anzahl korrekt wiedergegebener Zahlensequenzen.

Die Exekutiven Funktionen wurden in Anlehnung an die drei Dimensionen Inhibition, Updating und Shifting von Miyake, Friedman, Emerson, Witzki, Howerter und Wager (2000) operationalisiert. Dazu wurde ein z-standardisierter Summenwert von drei Testaufgaben berechnet, die bei Röthlisberger, Neuenschwander, Michel und Roebers (2010) detailliert beschrieben werden. Zur Messung der Inhibition diente die Früchte-Gemüse-Stroop-Aufgabe gemäß Archibald und Kerns (1999) (abhängige Variable: Interferenzmaß nach Archibald \& Kerns, 1999, Verrechnung der benötigten Zeiten in Sekunden unter vier verschiedenen Bedingungen, kein festgelegter Maximalwert), zur Messung des Updatings die Aufgabe Farbenspanne rückwärts (abhängige Variable: Anzahl richtiger Antworten, maximal 18) und zur Messung des Shiftings die Aufgabe Kognitive Flexibilität (abhängige Variable: Prozentanteil der richtigen Antworten unter den Switch-Bedingungen, maximal 100).

Zur Erhebung der mathematischen Vorläuferfertigkeiten wurden die zwei Aufgaben Würfelaugen zählen und Einfache Addition eingesetzt. Bei der Aufgabe Würfelaugen zählen wurden auf einem Blatt insgesamt 20 Würfel präsentiert. Die Kinder mussten möglichst schnell die Augenzahl der einzelnen Würfel nennen (adaptiert nach Krajewski, 2003). Bei der Aufgabe Einfache Addition mussten die Kinder jeweils die Summe der Augenzahl 
zweier Würfel (angeordnet in drei Reihen an je drei Würfelpaare) nennen (adaptiert nach Krajewski, Schneider \& Nieding, 2008). Als abhängige Variable beider Teilaufgaben dienten die benötigten Sekunden, die für das Maß der mathematischen Vorläuferfertigkeiten aufsummiert wurden.

Zwei Aufgaben wurden zur Erhebung der schriftsprachlichen Vorläuferfertigkeiten eingesetzt. Die erste Aufgabe Objekte benennen bestand darin, dass die Kinder 40 verschiedene Objekte so schnell als möglich benennen sollten (Di Filippo, Zoccolotti \& Ziegler, 2008). Diese in der deutschen Sprache ein- oder zweisilbigen Objekte wurden den Kindern als Abbildungen auf einem Blatt in fünf Reihen von je acht Objekten präsentiert. Als abhängige Variable galt die für die Benennung aller Objekte benötigte Zeit in Sekunden. Bei der zweiten Aufgabe Tierarten aufzählen wurden die Kinder gebeten, innerhalb von 1 Minute möglichst viele verschiedene Tierarten zu nennen (Bialystok, Luk \& Craik, 2008; Milner, 1964). Die abhängige Variable war die Anzahl korrekt genannter Tierarten, welche ohne Wiederholungen innerhalb von 1 Minute genannt wurden. Die Werte für die schriftsprachlichen Vorläuferfertigkeiten ergaben sich aus den aufgrund unterschiedlicher Metriken z-standardisierten Variablen von Objekte benennen und Tierarten aufzählen.

Ergebnisse

\section{Faktorenstruktur der Selbstkonzeptskala}

Um mehr über die Struktur des Selbstkonzepts von Kindergarten- bzw. Schulkindern zu erfahren, wurden die 10 Themenbereiche der Selbstkonzeptskala explorativen Faktorenanalysen unterzogen. Diese Berechnungen wurden zur Entdeckung allfälliger altersbedingter Strukturunterschiede im Selbstkonzept für Kindergarten- und Schulkinder getrennt durchgeführt. Tabelle 1 zeigt die resultierenden Faktorladungen aller erfragten Themenbereiche, getrennt für die beiden Altersgruppen.

\section{Tabelle 1 - hier einfügen}

Als Lösungen ergaben sich in beiden Berechnungen drei Faktoren mit Eigenwerten über dem Kaiserkriterium von 1, die zusammen 57 \% bzw. 58 \% der Varianz erklärten. Damit ließen sich anhand der Faktorenanalyse die erwarteten drei Bereiche unterscheiden: das leistungsbezogene Vorakademische Selbstkonzept (VaSK), das nicht leistungsbezogene Soziale Selbstkonzept (SoSK) sowie das Spielbezogene Selbstkonzept (SpSK). Im Folgenden werden deshalb anstelle der einzelnen Themenbereiche diese drei Komponenten als abhängige Variablen des Selbstkonzepts für beide Altersgruppen verwendet. Deskriptive Angaben zu den drei Komponenten sind der Tabelle 2 zu entnehmen. 
Tabelle 2 - hier einfügen

\section{Geschlechtsunterschiede}

Einfaktorielle Varianzanalysen zeigten, dass Knaben sich im SoSK signifikant und im SpSK tendenziell höher einschätzten als Mädchen, $F(1,292)=5.02, p<.05, \eta_{\mathrm{p}}{ }^{2}$ $($ partielles Eta-Quadrat $)=.02$ bzw. $F(1,292)=2.86, p<.10, \eta_{\mathrm{p}}{ }^{2}=.01 . \mathrm{Im}$ VaSK manifestierten sich die Geschlechtsunterschiede nur in separaten Analysen der zwei Altersgruppen: Bei den Kindergartenkindern erreichten die Mädchen höhere Werte als die Jungen, $F(1,107)=5.50, p<.05, \eta_{\mathrm{p}}{ }^{2}=.03$, bei den Schulkindern hingegen wiesen die Knaben ein höheres Selbstkonzept auf, $F(1,184)=5.38, p<.05, \eta_{\mathrm{p}}{ }^{2}=.03$. Die Mädchen zeigten darüber hinaus bessere Leistungen in den Exekutiven Funktionen, $F(1,289)=4.06$, $p<.05, \eta_{\mathrm{p}}{ }^{2}=.01$. Da diese Geschlechtsunterschiede relativ klein sind (vgl. Effektstärken $\eta_{\mathrm{p}}{ }^{2}$ ) und nicht im Fokus dieses Beitrages stehen, werden die Daten von Jungen und Mädchen im Folgenden gemeinsam ausgewertet.

Ausprägung des Selbstkonzepts

Tabelle 2 zeigt die deskriptiven Werte der drei Selbstkonzeptkomponenten, der Differenziertheitsmaße sowie der bereichsübergreifenden kognitiven Kompetenzen und der schulischen Vorläuferfertigkeiten - jeweils getrennt für Kindergarten- und Schulkinder. Bereits ein Blick auf die Mittelwerte der drei Selbstkonzeptkomponenten in Tabelle 2 zeigt, dass sich Kinder in der Schuleingangsphase im Durchschnitt auf den obersten Rängen der Klasse positionierten $(M=17.6$ bis $M=20.5$, bei einem Maximalwert von 25$)$. Um statistisch zu überprüfen, ob Kinder in der Schuleingangsphase überhöhte Selbstkonzeptwerte aufweisen, wurden Ein-Stichproben-T-Tests in Referenz zum Testwert 13 berechnet, was dem hypothetisch ,realistischen“ Mittelwert der Skala entspricht. Bei Kindergarten- und Schulkindern lagen die Mittelwerte aller 10 erfragten Themenbereiche des Selbstkonzepts hochsignifikant $(p<.001)$ über dem Skalenmittelwert von 13; die einzige Ausnahme bildete „Wörter lesen und schreiben“ bei den Kindergartenkindern, $M=14.1$, $t(107)=1.20, p>.05$. Um einen Unterschied der Selbstkonzeptausprägung zwischen Kindergarten- und Schulkindern festzustellen, wurden einfaktorielle Varianzanalysen durchgeführt. Die Ergebnisse der Mittelwertvergleiche sowie die Effektstärken sind in den letzten beiden Spalten in Tabelle 2 zu sehen. Einem bereichsspezifischen Veränderungsmuster entsprechend ergaben sich Unterschiede der Selbstkonzeptausprägung zwischen Kindergarten- und Schulkindern: Während das VaSK vom Kindergarten in die 
Schule relativ stark anstieg, nahmen das SoSK und das SpSK vom Kindergarten in die Schule leicht $\mathrm{ab}$.

Ausdifferenzierung des Selbstkonzepts

Zur Überprüfung, ob Schulkinder im Vergleich zu Kindergartenkindern tatsächlich eine stärkere Selbstkonzeptausdifferenzierung aufweisen, wurden zwei Analysen durchgeführt. Einerseits wurden die Interkorrelationen zwischen den Selbstkonzeptkomponenten innerhalb der beiden Altersgruppen miteinander verglichen. Dabei wurde erwartet, dass die Interkorrelationen zwischen den drei Selbstkonzeptkomponenten aufgrund der stärkeren Bereichsspezifität bei den Schulkindern geringer ausfallen würden als bei den Kindergartenkindern. Die Interkorrelationen lagen jedoch bei den Kindergartenkindern zwischen $r=.27$ und $r=.34(p<.05)$ und bei den Schulkindern zwischen $r=.24$ und $r=.36(p<.05)$, also in einem sehr ähnlichen Größenbereich (vgl. auch erste drei Zeilen bzw. Spalten in Tabelle 3).

Tabelle 3 - hier einfügen

Andererseits wurde angenommen, dass sich eine verstärkte Ausdifferenzierung insbesondere in einer zunehmenden Anzahl unterschiedlicher Positionen zeigen würde, welche ein Kind über alle erfragten Themenbereiche der Selbstkonzeptskala hinweg wählt. Dementsprechend wurde erwartet, dass Schulkinder ein größeres DM aufweisen als Kindergartenkinder. Tabelle 2 zeigt, dass Schulkinder tatsächlich signifikant mehr Rangpositionen für ihre Selbsteinschätzung nutzten als Kindergartenkinder. Diese Unterschiede zeigten sich sowohl über alle 10 Themenbereiche als auch separat über die vier vorakademischen $\left(\mathrm{DM}_{\text {vorakademisch }}\right)$ und die vier sozialen Themenbereiche $\left(\mathrm{DM}_{\text {sozial }}\right)$ hinweg. Des Weiteren lässt sich Tabelle 3 entnehmen, dass das DM mit der Höhe aller drei Selbstkonzeptkomponenten bedeutsam negativ korrelierte: Je tiefer ein Kind sich selbst einschätzte, umso mehr Positionen wählte es auf der Selbstkonzeptskala. Um diese Konfundierung des DM mit der Selbstkonzepthöhe zu kontrollieren, wurde zusätzlich eine Kovarianzanalyse (unabhängige Variable Altersgruppe: Kindergarten- vs. Schulkinder) mit der abhängigen Variablen DM und den drei Selbstkonzeptkomponenten VaSK, SoSK und SpSK als Kovariaten durchgeführt. Obwohl sich zwei Kovariaten als bedeutsam erwiesen (SoSK: $F(1,288)=28.98, p<.001$; SpSK: $F(1,288)=32.95, p<.001)$, blieb der Alterseffekt für das DM bestehen, $F(1,288)=37.21, p<.001, \eta_{\mathrm{p}}{ }^{2}=.11$. Während der Vergleich der Interkorrelationen zwischen den drei Selbstkonzeptkomponenten also keinen Hinweis auf eine zunehmende Ausdifferenzierung des Selbstkonzepts vom Kindergarten in die Schule 
lieferten, zeigte sich im DM ein konsistenter, bereichsunabhängiger Unterschied zwischen den Kindergarten- und Schulkindern.

\section{Zusammenhänge zwischen Selbstkonzeptvariablen und kognitiven Leistungsindikatoren}

Um Genaueres über die Zusammenhänge zwischen dem Selbstkonzept und den kognitiven Leistungsindikatoren aussagen zu können, wurden Pearson-Korrelationen zwischen den verschiedenen Variablen berechnet. Diese Korrelationen sind in Tabelle 3 getrennt für die beiden Altersgruppen (unterhalb bzw. oberhalb der Diagonalen) dargestellt. Es zeigten sich, wie erwartet, bedeutsame Zusammenhänge zwischen dem leistungsbezogenen VaSK und den Maßen der schulischen Vorläuferfertigkeiten. Je besser sich also die Kinder in ihren (vor)akademischen Fähigkeiten einschätzten, umso weniger Zeit brauchten sie für die Zähl- und Additionsaufgaben (mathematische Vorläuferfertigkeiten) und umso mehr Tierarten und Objekte konnten sie nennen (schriftsprachliche Vorläuferfertigkeiten). Entgegen unseren Erwartungen waren diese Zusammenhänge bei den Kindergartenkindern deskriptiv größer als bei den Schulkindern, interferenzstatistisch unterschieden sich die z-transformierten Korrelationswerte jedoch nicht voneinander (mathematische Vorläuferfertigkeiten: $r=-.16$ [Schulkinder], $r=-.22$ [Kindergartenkinder], $\mathrm{z}_{\mathrm{Differenz}}=0.52$, n.s., schriftsprachliche Vorläuferfertigkeiten: $r=.14$ [Schulkinder], $r=.21$ [Kindergartenkinder], $\mathrm{z}_{\text {Differenz }}=0.63$, n.s. $)$. Während sich bei den Kindergartenkindern diese signifikanten Zusammenhänge wie erwartet nur zwischen VaSK und schulischen Vorläuferfertigkeiten, nicht aber zwischen VaSK und bereichsübergreifenden kognitiven Kompetenzmaßen zeigten, war das Muster bei den Schulkindern weniger eindeutig. Es zeigten sich bei den Schulkindern auch tendenziell signifikante Zusammenhänge zwischen VaSK und Fluider Intelligenz bzw. Exekutiven Funktionen.

Zwischen dem nicht leistungsbezogenen SoSK und den kognitiven Leistungsindikatoren blieben bedeutsame Zusammenhänge aus. Bei den Schulkindern nicht aber bei den Kindergartenkindern - zeigten sich zwischen dem SpSK und drei von fünf kognitiven Leistungsindikatoren tendenzielle Zusammenhänge: Je besser sich die Schulkinder im „Puzzle zusammensetzen“ und „Memory spielen“ einschätzten, umso schlechter waren ihre Leistungen im Intelligenztest, in den Exekutiven Funktionen und in den mathematischen Vorläuferfertigkeiten.

Ein auffallendes Zusammenhangsmuster ergab sich zwischen dem DM und den kognitiven Indikatoren, jedoch mit Altersunterschieden: Während Kindergartenkinder mit 
einem höheren DM bessere Werte in der Fluiden Intelligenz und in den Exekutiven Funktionen aufwiesen, zeigten Schulkinder mit einem höheren DM gleichzeitig bessere Leistungen im phonologischen Arbeitsgedächtnis, den Exekutiven Funktionen und den mathematischen Vorläuferfertigkeiten.

\section{Diskussion}

In der vorliegenden Studie wurde das Selbstkonzept von 6- bis 8-jährigen Kindern $(N=297)$ untersucht. Von Interesse war dabei, welche Ausprägung und welche Struktur das Selbstkonzept aufweist und welche Zusammenhänge zwischen verschiedenen Selbstkonzeptkomponenten und schulischen Vorläuferfertigkeiten sowie bereichsübergreifenden kognitiven Kompetenzen bestehen. Ein Vergleich zwischen Kindergartenkindern und Kindern der ersten Grundschulklasse sollte Hinweise auf frühe Entwicklungsveränderungen in den drei Aspekten liefern.

Einige der bisherigen Forschungsbefunde, die vor allem aus dem englischsprachigen Raum stammen, konnten anhand der Studie repliziert werden. So bestätigte sich beispielsweise in dieser Untersuchung, in welcher ein neues Messinstrument zur Erhebung des Selbstkonzepts eingesetzt wurde, dass sich eine leistungsbezogene (VaSK) und eine nicht leistungsbezogene (SoSK) Selbstkonzeptkomponente unterscheiden lassen (vgl. Ergebnisse anhand der PSPCSA von Harter \& Pike, 1984). Außerdem neigten die Kinder zu stark überhöhten Selbsteinschätzungen. Der Regel der Bereichsspezifität entsprechend ließen sich ebenfalls bedeutsame Zusammenhänge zwischen dem leistungsbezogenen Selbstkonzept VaSK und den schulischen Vorläuferfertigkeiten nachweisen; Hinweise auf eine Zunahme dieser Zusammenhänge mit dem Alter blieben jedoch aus. Hinsichtlich früher Entwicklungsveränderungen der Selbstkonzeptstruktur wurden nach herkömmlichen Berechnungen (d. h. Interkorrelationen zwischen Selbstkonzeptkomponenten und Faktorenanalysen) keine Unterschiede zwischen Kindergarten- und Schulkindern gefunden. Es ergaben sich hingegen Altersunterschiede in der Ausprägung des Selbstkonzepts: Beim leistungsbezogenen VaSK war eine starke Zunahme und beim nicht leistungsbezogenen SoSK eine leichte Abnahme vom Kindergarten- ins Schulalter zu verzeichnen. Dieses bereichsspezifische Veränderungsmuster in der frühen Entwicklung wurde bereits in Längsschnittsstudien gefunden (z. B. Helmke, 1998; Mantzicopoulos, 2006). Die Zunahme des leistungsbezogenen Selbstkonzepts wurde in der Literatur mit einer „Schonphase“ hinsichtlich Leistungsrückmeldungen in der ersten Klasse in Verbindung gebracht (Helmke, 1998). Der speziell nach dem Schuleintritt stattfindende massive objektive 
Kompetenzzuwachs, den die Kinder gerade in den untersuchten vorakademischen Bereichen wie z. B. „Buchstaben kennen“ und „zählen“ erleben, könnte ebenfalls zu diesem zunehmenden subjektiven Kompetenzbewusstsein beitragen. Dies würde die Annahme bestätigen, dass internal-temporale Vergleiche zur Entstehung insbesondere des leistungsbezogenen Selbstkonzepts beitragen (Helmke, 1992; Nicholls, 1984; Schöne, Dickhäuser, Spinath \& Stiensmeier-Pelster, 2003).

Die vorliegende Studie lieferte zudem zwei neue Befunde zur frühen Selbstkonzeptentwicklung: die Komponente des Spielbezogenen Selbstkonzepts und das Differenziertheitsmaß. Diese zwei Befunde sollen im Folgenden hinsichtlich ihrer Bedeutsamkeit und ihres Nutzens kritisch diskutiert werden.

\section{Spielbezogenes Selbstkonzept}

Unsere Untersuchung hatte zum Ziel, die Selbsteinschätzungen in Bereichen zu erfassen, welche für junge Kinder insbesondere im (vor)schulischen Alltag bedeutungsvoll sind. Die adaptierte Selbstkonzeptskala beinhaltete deshalb neben typischerweise eher schulischen Leistungsbereichen und dem Bereich der Gleichaltrigenbeziehungen in der Klassengemeinschaft auch Themen wie „Puzzle zusammensetzen“ und „Memory spielen“. Faktorenanalytisch ließ sich aus diesen zwei letzten Themenbereichen für beide Altersgruppen eine separate spielbezogene Komponente des Selbstkonzepts extrahieren. Dieses Ergebnis gilt es vorerst jedoch mit Vorsicht zu interpretieren und es bedarf einer Replikation, um auszuschließen, dass in der vorliegenden Untersuchung lediglich die beiden Themenbereiche, welche zu den anderen zwei Komponenten weniger gut passten, einen dritten, gemeinsamen Faktor ergaben. Inwiefern es sich tatsächlich um ein spielbezogenes Selbstkonzept handelt, müssten weitere Untersuchungen beispielsweise dadurch überprüfen, dass die Selbstkonzeptskala durch zusätzliche Spielaktivitäten erweitert würde. Falls sich die Komponente auch in anderen Untersuchungen bestätigen ließe, würde sich des Weiteren die Frage stellen, ob das Spielbezogene Selbstkonzept in einem altersangepassten Modell von Shavelson et al. (1976) eher dem leistungsbezogenen oder dem nicht leistungsbezogenen Aspekt des Selbst zugeordnet werden sollte. Im ursprünglichen Modell entspricht der leistungsbezogene Bereich dem akademischen Selbstkonzept und der nicht leistungsbezogene Bereich subsumiert die emotionale, die physische und die soziale Komponente des Selbst (Shavelson et al., 1976). Unsere Ergebnisse bezüglich der frühen Entwicklungsveränderungen in der Ausprägung und der Zusammenhänge zwischen Spielbezogenem Selbstkonzept und kognitiven Indikatoren sprechen hier eher für eine 
Ähnlichkeit mit dem nicht leistungsbezogenen Selbstkonzept (SoSK) als mit dem leistungsbezogenen Selbstkonzept (VaSK).

Differenziertheitsmaß

Während sich durch die herkömmlichen Berechnungsmethoden keine Hinweise auf Altersunterschiede in der Ausdifferenzierung des Selbstkonzepts ergaben, erwies sich das Differenziertheitsmaß als neue, entwicklungssensitive Größe. Es zeigte sich nämlich, dass Schulkinder im Vergleich zu Kindergartenkindern eine größere Variation an Rangpositionen auf der Selbstkonzeptskala wählten und zwar unabhängig davon, ob sie sich im leistungsbezogenen ( $\left.\mathrm{DM}_{\text {vorakademisch }}\right)$ oder im nicht leistungsbezogenen Bereich $\left(\mathrm{DM}_{\text {sozial }}\right)$ einschätzten. Diese Variation in den Rangpositionen schien wiederum mit der Selbstkonzepthöhe verbunden zu sein: In beiden Altersgruppen wurden bedeutsame, negative Zusammenhänge ( $r=-.19$ bis $r=-.50)$ zwischen dem Differenziertheitsmaß und der Höhe der drei Selbstkonzeptkomponenten gefunden. Je mehr Variation Kinder in ihren Selbstpositionierungen aufwiesen, umso tiefer fiel ihr Selbstkonzept aus. In diesem negativen Zusammenhang könnte man ein Artefakt vermuten, das sich lediglich daraus ergab, dass die Kinder ihre überhöhten Selbsteinschätzungen auf der Selbstkonzeptskala nur hin zu tieferen Positionen variieren konnten. Die Altersunterschiede im Differenziertheitsmaß, welche selbst nach Kontrolle der Höhe des Selbstkonzepts bestehen blieben, weisen jedoch darauf hin, dass ein isolierter Effekt der gemessenen Ausdifferenzierung unabhängig von der Selbstkonzeptausprägung messbar ist. In den vorliegenden Daten findet sich also der Hinweis auf einen Ausdifferenzierungsprozess, der zwar zu einem relativ frühen Zeitpunkt in der Entwicklung relativ eng mit der Ausprägung des Selbstkonzepts verbunden ist, dennoch aber als distinktes Phänomen erfasst werden kann.

Eine weitere Eigenschaft dieser frühen Ausdifferenzierung zeigt sich in den bedeutsamen Korrelationen zwischen dem Differenziertheitsmaß und den kognitiven Leistungsindikatoren. Ein Anstieg im Differenziertheitsmaß bedeutet, zunehmend über ein persönliches Profil zu verfügen, welches nicht nur Stärken, sondern auch relative Schwächen beinhaltet. Genau diese Fähigkeit bringt Harter (1999) in ihrem Entwicklungsmodell des Selbstkonzepts mit der Stufentheorie der kognitiven Entwicklung von Piaget und Inhelder (1955, übers.1977) in Verbindung. Das uni-dimensionale Denken der präoperationalen Phase, das im Kindergartenalter vorherrschend ist, wird im Schulalter vom bi-dimensionalen Denken der operationalen Phase abgelöst (Harter, 1998). Die bedeutsamen Korrelationen 
zwischen dem Differenziertheitsmaß und den Exekutiven Funktionen, dem phonologischem Arbeitsgedächtnis und der Fluiden Intelligenz könnten genau auf diesen Zusammenhang zwischen dem Grad der Ausdifferenzierung des Selbstkonzepts und der kognitiven Reife hindeuten.

Bei einem Vergleich der Zusammenhänge, die sich zwischen dem VaSK und den kognitiven Leistungsindikatoren einerseits und zwischen dem Differenziertheitsmaß und diesen Indikatoren andererseits ergeben, fällt zudem auf, dass die Ausprägung des Selbstkonzepts als absolute Größe zwar charakteristisch mit den bereichsspezifischen Vorläuferfertigkeiten korreliert, dass jedoch das Differenziertheitsmaß als relative Größe des Selbstkonzepts ebenfalls vergleichbare, teilweise sogar höhere Zusammenhänge mit verschiedenen kognitiven Leistungsindikatoren aufweisen kann. Die Befunde sprechen insgesamt dafür, dass dieses entwicklungssensitive, normalverteilte, der Selbstkonzeptskala inhärente Differenziertheitsmaß einen potenziell wichtigen Reifeprozess in der Selbstkonzeptentwicklung widerspiegelt und speziell bei Untersuchungen des Selbstkonzepts im Schuleingangsalter berücksichtigt werden sollte. Allerdings lässt sich nicht völlig ausschließen, dass kognitiv leistungsfähigere Kinder lediglich besser mit dem Format der Selbstkonzeptskala umgehen konnten als weniger leistungsfähige Kinder. Um spezifischere Aussagen über das Differenziertheitsmaß machen zu können, sollten weitere Untersuchungen folgen, welche beispielsweise den gefundenen Alterseffekt längsschnittlich zu bestätigen versuchen.

Obschon in der vorliegenden Untersuchung sowohl einige erwartungskonforme Ergebnisse repliziert als auch neue Erkenntnisse erzielt werden konnten, birgt die Studie auch Limitationen, vor allem im Hinblick auf die eingesetzte Selbstkonzeptskala. So wurde mit dem Messinstrument bewusst eine soziale Bezugsnorm induziert; einerseits, weil frühere Forschungsbefunde gezeigt haben, dass soziale Vergleiche wichtige Informationen für die Selbstkonzeptgenese junger Kinder darstellen und andererseits, um den Kindern die eigene Einschätzung zu erleichtern. Der soziale Vergleich führt jedoch oft zu starken Bezugsgruppeneffekten, sodass typischerweise zwei SchülerInnen mit identischem Leistungsprofil in unterschiedlich leistungsstarken Klassen nicht ein gleich hohes Selbstkonzept aufweisen (sog. Fischteich-Effekt; Marsh, 1987). Es ist folglich anzunehmen, dass die individuelle Zusammensetzung der jeweiligen Klassen einen Einfluss darauf hatte, wo sich Kinder innerhalb ihrer Gruppe positionierten. Diese Tatsache müsste darum durch eine klassenweise Berechnung der Korrelationen zwischen Selbstkonzept und kognitiven 
Indikatoren berücksichtigt werden. Ein solches mehrebenenanalytisches Vorgehen war aber auf der Basis unserer Stichprobe nicht möglich, weil diese aus einer laufenden Längsschnittstudie stammte und pro Klasse durchschnittlich lediglich Daten von vier Kindern vorlagen. Die Kinder haben also vermutlich ihre Selbsteinschätzung im Vergleich zu einer Gruppe vorgenommen, zu der uns kaum Daten bekannt waren. In Zukunft wäre sicherzustellen, dass beim Einsatz der Selbstkonzeptskala möglichst viele Daten zur Vergleichsgruppe vorliegen. Ein weiterer Kritikpunkt der Studie besteht darin, dass zwei Gruppen verglichen wurden, welche sich sowohl hinsichtlich ihres Alters als auch ihres institutionellen Kontextes (Kindergarten vs. Schule) unterschieden. Alters- und Beschulungseffekte sind hier konfundiert und ihre isolierten Einflüsse auf die Selbstkonzeptentwicklung könnten erst in Studien, welche ein sogenanntes Cutoff-Design (nach Bisanz, Morrison \& Dunn, 1995) verwenden, näher bestimmt werden.

Trotz dieser Einschränkungen kann die vorliegende Untersuchung als Beitrag zu einem aktuellen Forschungsanliegen betrachtet werden, welches zum Ziel hat, motivationale Entwicklungsverläufe bereits von der Vorschulzeit an zu beobachten (Mantzicopoulos, 2006) und die Schuleingangsphase nicht nur für den kognitiven, sondern auch für den affektiv-motivationalen Entwicklungsbereich möglichst positiv zu gestalten. 
Literaturverzeichnis

Anthony, J. L., Williams, J. M., McDonald, R. \& Francis, D. J. (2007). Phonological processing and emergent literacy in younger and preschool children. Annals of Dyslexia, 57, 113-137.

Archibald, S. J. \& Kerns, K. A. (1999). Identification and description of new tests of executive functioning in children. Child Neuropsychology, 5, 115-129.

Asendorpf, J. B. \& van Aken, M. A. G. (1993). Deutsche Versionen der Selbstkonzeptskalen von Harter. Zeitschrift für Entwicklungspsychologie und Pädagogische Psychologie, $25,64-86$.

Aunola, K., Leskinen, E., Onatsu-Arvilommi, T. \& Nurmi, J.-E. (2002). Three methods for studying developmental change: A case of reading skills and self-concept. British Journal of Educational Psychology, 72, 343-364.

Best, J. R., Miller, P. H. \& Jones, L. L. (2009). Executive functions after age 5: Changes and correlates. Developmental Review, 29, 180-200.

Beutel, S.-I. \& Hinz, R. (2008). Schulanfang im Wandel-Selbstkonzepte der Kinder als pädagogische Aufgabe. Berlin: LIT Verlag.

Bialystok, E., Luk, G. \& Craik, F. (2008). Cognitive control and lexical access in younger and older bilinguals. Journal of Experimental Psychology: Learning, Memory, and Cognition, 34, 859-873.

Bisanz, J., Morrison, F. J. \& Dunn, M. (1995). Effects of age and schooling on the acquisition of elementary quantitative skills. Developmental Psychology, 31, 221236.

Blair, C. \& Razza, R. P. (2007). Relating effortful control, executive function, and false belief understanding to emerging math and literacy ability in kindergarten. Child Development, 78, 647-663.

Bouffard, T., Marcoux, M.-F., Vezeau, C. \& Bordeleau, L. (2003). Changes in selfperceptions of competence and intrinsic motivation among elementary schoolchildren. British Journal of Educational Psychology, 73, 171-186.

Brown, L., Sherbenou, R. J. \& Johnson, S. K. (1997). Test of nonverbal intelligence: A language-free measure of cognitive ability ( $3^{\text {th }}$ ed.). Austin: Pro-Ed. 
Buff, A., Nakamura, Y., Hollenweger, J. \& Achermann, E. (2005). Selbstwahrnehmung bei Schuleintritt. In U. Moser, M. Stamm \& J. Hollenweger (Hrsg.), Für die Schule bereit? Oberentfelden: Sauerländer.

Cimeli, P., Neuenschwander, R., Röthlisberger, M. \& Roebers, C. M. (2010).

Längsschnittstudie zum Selbstkonzept bei Kindern im Alter von 5 bis 7 Jahren [Abstract]. In F. Petermann \& U. Koglin (Hrsg.), 47. Kongress der Deutschen Gesellschaft für Psychologie. (S.25, 26). Lengerich: Pabst Science Publishers.

Daseking, M., Oldenhage, M. \& Petermann, F. (2008). Der Übergang vom Kindergarten in die Grundschule - Eine Bestandesaufnahme. Psychologie in Erziehung und Unterricht-Zeitschrift für Forschung und Praxis, 2, 84-99.

Di Filippo, G., Zoccolotti, P. \& Ziegler, J. C. (2008). Rapid naming deficits in dyslexia: A stumbling block for the perceptual anchor theory of dyslexia. Developmental Science, 11, F40-F47.

Dickhäuser, O. \& Galfe, E. (2004). Besser als..., schlechter als...: Leistungsbezogene Vergleichsprozesse in der Grundschule. Zeitschrift für Entwicklungspsychologie und Pädagogische Psychologie, 36, 1-9.

Eccles, J. S. \& Wigfield, A. (2002). Motivational beliefs, values, and goals. Annual Review of Psychology, 53, 109-132.

Filipp, S. H. (2006). Kommentar zum Themenschwerpunkt. Entwicklung von Fähigkeitsselbstkonzepten. Zeitschrift für Entwicklungspsychologie und Pädagogische Psychologie, 20, 65-72.

Filipp, S. H. (Hrsg.). (1984). Selbstkonzept-Forschung: Probleme, Befunde, Perspektiven (Bd. 2). Stuttgart: Klett-Cotta.

French, B. F. \& Mantzicopoulos, P. (2007). An examination of the first/ second- grade form of the pictoral scale of perceived competence and social acceptance: Factor structure and stability by grade and gender across groups of economically disadvantaged children. Journal of School Psychology, 45, 311-331.

Guay, F., Marsh, H. W. \& Boivin, M. (2003). Academic self-concept and academic achievement: Developmental perspectives on their causal ordering. Journal of Educational Psychology, 95, 124-136.

Hansford, B. C. \& Hattie, J. A. (1982). The relationship between self and achievement/ performance measures. Review of Educational Research, 52, 123-142. 
Harter, S. (1998). The development of self-representations. In W. Damon \& N. Eisenberg (Eds.), Handbook of Child Psychology: Social, Emotional, and Personality Development (pp. 553-617). New York: Wiley.

Harter, S. (1999). The construction of the self: A developmental perspective. New York: Guilford Press.

Harter, S. \& Pike, R. (1984). The pictorial scale of perceived competence and social acceptance for young children. Child Development, 55, 1969-1982.

Helmke, A. (1992). Selbstvertrauen und schulische Leistungen. Göttingen: Hogrefe.

Helmke, A. (1998). Vom Optimisten zum Realisten? Zur Entwicklung des Fähigkeitskonzeptes vom Kindergarten bis zur 6. Klassenstufe. In F. E. Weinert (Hrsg.), Entwicklung im Kindesalter (S.115-132). Weinheim: Beltz Psychologie Verlags Union.

Hughes, J. N., Dyer, N., Luo, W. \& Kwok, O.-M. (2009). Effects of peer academic reputation on achievement in academically at-risk elementary students. Journal of Applied Developmental Psychology, 30, 182-194.

Jacobs, J. E., Lanza, S., Osgood, D. W., Eccles, J. S. \& Wigfield, A. (2002). Changes in children`s self-competence and values: Gender and domain differences across grades one through twelve. Child Development, 73, 509-527.

Köller, O. (2004). Konsequenzen von Leistungsgruppierungen. Münster: Waxmann.

Krajewski, K. (2003). Vorhersage von Rechenschwäche in der Grundschule. Hamburg: Verlag Dr. Kovac.

Krajewski, K. \& Schneider, W. (2006). Mathematische Vorläuferfertigkeiten im Vorschulalter und ihre Vorhersagekraft für die Mathematikleistungen bis zum Ende der Grundschulzeit. Psychologie in Erziehung und Unterricht, 53, 246-262.

Krajewski, K. \& Schneider, W. (2009). Exploring the impact of phonological awareness, visual-spatial working memory, and preschool quantity-number competencies on mathematics achievement in elementary school: Findings from a 3-year longitudinal study. Journal of Experimental Child Psychology, 103, 516-531.

Krajewski, K., Schneider, W. \& Nieding, G. (2008). Zur Bedeutung von Arbeitsgedächtnis, Intelligenz, phonologischer Bewusstheit und früher Mengen-Zahlen-Kompetenz beim Übergang vom Kindergarten in die Grundschule. Psychologie in Erziehung und Unterricht, 2, 100-113. 
Mantzicopoulos, P. (2006). Younger children`s changing self-concept: Boys and girls from preschool through second grade. The Journal of Genetic Psychology, 167, 289-308.

Mantzicopoulos, P., French, B. F. \& Maller, S. J. (2004). Factoral structure of the pictoral scale of perceived competence and social acceptance with two pre-elementary samples. Child Development, 75, 1214-1228.

Marsh, H. W. (1987). The big-fish-little-pond effect on academic self-concept. Journal of Educational Psychology, 79, 280-295.

Marsh, H. W. \& Ayotte, V. (2003). Do multiple dimensions of self-concept become more differentiated with age? The differential distinctiveness hypothesis. Journal of Educational Psychology, 95, 687-706.

Marsh, H. W., Craven, R. G. \& Debus, R. (1991). Self-concepts of young children 5 to 8 years of age: Measurement and multidimensional structure. Journal of Educational Psychology, 83, 377-392.

Marsh, H. W., Ellis, L. A. \& Craven, R. G. (2002). How do preschool children feel about themselves? Unraveling measurement and multidimensional self-concept structure. Developmental Psychology, 38, 376-393.

Marsh, H. W. \& Hocevar, D. (1985). Application of confirmatory factor analysis to the study of self-concept: First- and higher order factor models and their invariance across groups. Psychological Bulletin, 97, 562-582.

Martschinke, S. \& Kammermeyer, G. (2003). Jedes Kind ist anders. Jede Klasse ist anders. Zeitschrift für Erziehungswissenschaft, 6, 257-275.

Meyer, W.-U. (1984). Das Konzept von der eigenen Begabung. Bern: Hans Huber.

Milner, B. (1964). Some effects of frontal-lobe lobectomy in man. In J. Warren \& K. Akert (Eds.), The frontal granular cortex and behaviour (pp. 313-331): New York: McGraw-Hill.

Miyake, A., Friedman, N. P., Emerson, M. J., Witzki, A. H., Howerter, A. \& Wager, T. D. (2000). The unity and diversity of executive functions and their contribution to complex frontal lobe tasks: A latent variable analysis. Cognitive Psychology, 41, 49100.

Näslund, J. C. \& Schneider, W. (1996). Kindergarten letter knowledge, phonological skills, and memory processes: Relative effects on early literacy. Journal of Experimental Child Psychology, 62, 30-59. 
Neuenschwander, R., Röthlisberger, M., Michel, E. \& Roebers, C. M. (2011). Unterschiede in ausgewählten Bereichen der Schulfähigkeit: Ein Vergleich von Kindergarten und einem neuen Schuleingangsmodell in der Schweiz. Psychologie in Erziehung und Unterricht, 58, 30-40.

Nicholls, J. G. (1978). The development of the concepts of effort and ability, perception of academic attainment, and the understanding that difficult tasks require more ability. Child Development, 49, 800-814.

Nicholls, J. G. (1984). Achievement motivation: Conceptions of ability, subjective experience, task choice, and performance. Psychological Review, 91, 328-346.

Niklas, F. \& Schneider, W. (in Druck). Die Anfänge geschlechtsspezifischer Leistungsunterschiede in mathematischen und schriftsprachlichen Kompetenzen. Zeitschrift für Entwicklungspsychologie und Pädagogische Psychologie.

Nurmi, J.-E. \& Aunola, K. (2005). Task-motivation during the first school years: A personoriented approach to longitudinal data. Learning and Instructions, 15, 103-122.

Organisation for Economic Co-operation and Development (2009). Ein guter Start ins Leben II: Frühkindliche Betreuung, Bildung und Erziehung. Zugriff am 6.12.2011 unter http://browse.oecdbookshop.org/oecd/pdfs/free/9106035e5.pdf

Piaget, J. \& Inhelder, B. (1977). Von der Logik des Kindes zur Logik des Heranwachsenden. (L. Bernard, Übers.) Olten: Walter-Verlag. (Original erschienen 1955: De la logique de l’enfant à la logique de l'adolescent)

Pior, R. (1998). Selbstkonzepte von Vorschulkindern- Empirische Untersuchungen zum Selbstkonzept sozialer Integration. Münster: Waxmann Verlag.

Renner, G. (2008). Das Selbstkonzept sozialer Integration bei Kindern in der jahrgangsgemischten Eingangsstufe. Unveröffentlichte Dissertation, Nürnberg: Friedrich-Alexander-Universität.

Robins, R. W. \& Beer, J. S. (2001). Positive illusions about the self: Short-term benefits and long term costs. Journal of Personality and Social Psychology, 80, 340-352.

Roebers, C. M. (2007). Entwicklung des Selbstkonzeptes. In M. Hasselhorn \& W. Schneider (Hrsg.), Handbuch der Entwicklungspsychologie (S. 381-391). Göttingen: Hogrefe. Roebers, C. M. \& Schneider, W. (1999). Self-concept and anxiety in immigrant children. International Journal of Behavioral Development, 23, 125-147. 
Rost, D. H. \& Sparfeldt, J. R. (2002). Facetten des schulischen Selbstkonzepts: Ein Verfahren zur Messung des differentiellen Selbstkonzepts schulischer Leistungen und Fähigkeiten (DISK-Gitter). Diagnostica, 48, 130-140.

Röthlisberger, M. \& Michel, E. (2009). Entwicklung und Evaluation eines Programms zur koordinativen Förderung von Kindern in Einschulungsklassen. Praxis der Kinderpsychologie und Kinderpsychiatrie, 58, 215-230.

Röthlisberger, M., Neuenschwander, R., Michel, E. \& Roebers, C. M. (2010). Exekutive Funktionen: Zugrundeliegende kognitive Prozesse und deren Korrelate bei Kindern im späten Vorschulalter. Zeitschrift für Entwicklungspsychologie und Pädagogische Psychologie, 42, 99-110.

Schöne, C., Dickhäuser, O., Spinath, B. \& Stiensmeier-Pelster, J. (2003). Das Fähigkeitsselbstkonzept und seine Erfassung. In J. Stiensmeier-Pelster \& F. Rheinberg (Hrsg.), Diagnostik von Motivation und Selbstkonzept (S. 3-14). Göttingen: Hogrefe.

Shavelson, R. J., Hubner, J. J. \& Stanton, G. C. (1976). Self-concept: Validation of construct interpretation. Review of Educational Research, 46, 407-441.

Shin, H., Bjorklund, D., F. \& Beck, E. F. (2007). The adaptive nature of children`s overestimation in a strategic memory task. Cognitive Development, 22, 197-212.

Spinath, B., Spinath, F. M., Harlaar, N. \& Plomin, R. (2006). Predicting school achievement from general cognitive ability, self-perceived ability, and intrinsic value. Intelligence, $34,363-374$.

Stipek, D. J. \& Daniels, D. H. (1988). Declining perceptions of competence: A consequence of changes in the child or in the educational environment? Journal of Educational Psychology, 80, 352-356.

Wagner, J. W. L. (1999). Soziale Vergleiche und Selbsteinschätzungen: Theorien, Befunde und schulische Anwendungsmöglichkeiten: Münster: Waxmann Verlag.

Wigfield, A. \& Eccles, J. S. (2002). The development of competence beliefs, expectancies for success, and achievement values from childhood through adolescence. In A. Wigfield \& J. S. Eccles (Eds.), Development of Achievement Motivation (pp. 91120). San Diego: Academic Press. 
Tabelle 1

Explorative Faktorenanalysen: Faktorladungen der 10 Themenbereiche des Selbstkonzepts, getrennt dargestellt für Kindergarten- und Schulkinder

\begin{tabular}{|c|c|c|c|}
\hline \multirow[b]{2}{*}{$\begin{array}{l}\text { Themenbereiche } \\
\text { Selbstkonzeptskala }\end{array}$} & \multicolumn{3}{|c|}{ Rotierte Faktorladungen } \\
\hline & $\begin{array}{c}\text { Vorakademisches } \\
\text { Selbstkonzept }\end{array}$ & $\begin{array}{c}\text { Soziales } \\
\text { Selbstkonzept }\end{array}$ & $\begin{array}{l}\text { Spielbezogenes } \\
\text { Selbstkonzept }\end{array}$ \\
\hline Buchstaben & $.83 / .70$ & $.11 /-.13$ & $-.20 / .16$ \\
\hline $\begin{array}{l}\text { Wörter lesen und } \\
\text { schreiben }\end{array}$ & $.76 / .76$ & $.30 / .01$ & $-.15 /-.04$ \\
\hline Zählen & $.64 / .66$ & $-.27 / .13$ & $.22 /-.02$ \\
\hline Rechnen & $.61 / .57$ & $-.08 / .08$ & $.35 / .04$ \\
\hline Daneben sitzen & $-.06 /-.01$ & $.77 / .80$ & $-.22 /-.06$ \\
\hline Spielaufforderung & $.07 /-.26$ & $.65 / .61$ & $.25 / .36$ \\
\hline Freunde haben & $-.10 / .13$ & $.58 / .79$ & $.37 /-.02$ \\
\hline Zusammen spielen & $.20 / .14$ & $.53 / .76$ & $.06 /-.02$ \\
\hline Puzzle & $-.05 / .16$ & $-.02 /-.02$ & $.83 / .78$ \\
\hline Memory & $.11 /-.01$ & $.23 / .02$ & $.60 / .84$ \\
\hline Eigenwerte & $2.34 / 2.19$ & $2.04 / 2.56$ & $1.77 / 1.88$ \\
\hline$\%$ Varianz & $28.94 / 16.38$ & $15.48 / 31.13$ & $12.31 / 11.00$ \\
\hline Cronbach`s Alpha & $.71 / .63$ & $.61 / .76$ & $.44 / .60$ \\
\hline
\end{tabular}

Anmerkungen. Faktorladungen $>.40$ sind fett gedruckt. Faktorladungen vor dem Schrägstrich $=$ Kindergartenkinder $(n=108)$, Faktorladungen hinter dem Schrägstrich $=$ Schulkinder $(n=185)$. Die Reihenfolge der Faktorladungen entspricht der Faktorenlösung für Kindergartenkinder. 
Tabelle 2

Deskriptive Werte zu Selbstkonzeptkomponenten, Differenziertheitsmaßen, bereichsübergreifenden kognitiven Kompetenzen und schulischen

Vorläuferfertigkeiten, einschließlich Mittelwertvergleichen ( $F$-Wert) zwischen Kindergarten- und Schulkindern

\begin{tabular}{|c|c|c|c|c|c|}
\hline & $\begin{array}{l}\text { Kindergarten- } \\
\text { kinder } \\
M(S D)\end{array}$ & $\begin{array}{l}\text { Schulkinder } \\
M(S D)\end{array}$ & Min. - Max. & $F$-Wert & $\eta_{\mathrm{p}}^{2}$ \\
\hline Vorakad. Selbstkonzept & $17.6(6.0)$ & $20.5(3.7)$ & $1.0-25.0$ & $25.66^{* *}$ & .08 \\
\hline Soziales Selbstkonzept & $20.3(4.5)$ & $18.8(4.8)$ & $1.0-25.0$ & $6.76^{*}$ & .02 \\
\hline Spielbez. Selbstkonzept & $20.3(4.9)$ & $18.5(5.2)$ & $1.0-25.0$ & $8.50 *$ & .03 \\
\hline Differenziertheitsmaß & $3.6(2.0)$ & $5.4(2.2)$ & $1.0-10.0$ & $49.87 * *$ & .15 \\
\hline $\mathrm{DM}_{\text {vorakademisch }}$ & $2.3(0.9)$ & $2.8(1.0)$ & $1.0-4.0$ & $23.92 * *$ & .08 \\
\hline $\mathrm{DM}_{\text {sozial }}$ & $2.1(1.0)$ & $2.8(1.0)$ & $1.0-4.0$ & $33.80 * *$ & .10 \\
\hline Fluide Intelligenz $^{a}$ & $9.5(3.8)$ & $13.4(5.2)$ & $0.0-34.0$ & $48.41 * *$ & .14 \\
\hline $\begin{array}{l}\text { Phonologisches } \\
\text { Arbeitsgedächtnis }{ }^{\text {a }}\end{array}$ & $4.4(2.1)$ & $6.2(1.9)$ & $2.0-7.0$ & $61.86^{* *}$ & .17 \\
\hline Exekutive Funktionen $^{\mathrm{b}}$ & $-.44(.69)$ & $.34(.60)$ & $-2.2-1.5$ & $101.04 * *$ & .26 \\
\hline $\begin{array}{l}\text { Mathematische } \\
\text { Vorläuferfertigkeiten }\end{array}$ & $91.0(29.6)$ & $53.2(23.1)$ & $21.1-205.6$ & $135.14 * *$ & .33 \\
\hline Würfelaugen zählen & $25.4(10.7)$ & $16.4(5.2)$ & $8.9-63.2$ & $95.67 * *$ & .25 \\
\hline Einfache Addition & $66.7(25.0)$ & $37.0(20.0)$ & $8.8-186.8$ & $114.24 * *$ & .29 \\
\hline $\begin{array}{l}\text { Schriftsprachliche } \\
\text { Vorläuferfertigkeiten }^{\text {b }}\end{array}$ & $-.58(1.6)$ & $.34(1.6)$ & $-6.6-4.7$ & $22.38 * *$ & .07 \\
\hline Objekte benennen & $69.8(17.8)$ & $64.3(17.1)$ & $18.2-158.0$ & $6.87 *$ & .02 \\
\hline Tierarten aufzählen & $11.5(4.6)$ & $14.4(4.4)$ & $3.0-29.0$ & $29.23 * *$ & .09 \\
\hline
\end{tabular}

Anmerkungen . Min. - Max. = Minimal- und Maximalwerte basierend auf den Mittelwerten der gesamten Stichprobe. $\eta_{\mathrm{p}}{ }^{2}=$ partielles Eta-Quadrat (Effektstärke).

vorakad. $=$ vorakademisch. spielbez. $=$ spielbezogen $. \mathrm{DM}_{\text {vorakademisch }}=$ Differenziertheitsmaß $(\mathrm{DM})$ berechnet aus den vier vorakademischen Themenbereichen. $\mathrm{DM}_{\text {sozial }}=\mathrm{DM}$ berechnet 
aus den vier sozialen Themenbereichen. ${ }^{a}$ Anzahl korrekter Antworten. ${ }^{b}$ z-standardisierte Summenwerte. ${ }^{\mathrm{c}}$ Benötigte Sekunden.

$* p<.05, * * p<.001$. 
Tabelle 3

Korrelationsmatrix für Kindergarten- und Schulkinder: Selbstkonzeptvariablen, bereichsübergreifende kognitive Kompetenzmaße und schulische Vorläuferfertigkeiten

\begin{tabular}{lcccccccccccccc}
\hline & VaSK & SoSK & SpSK & DM & FI & PA & EF & MV & SV \\
\hline Vorakademisches Selbstkonzept (VaSK) & - & $.24^{*}$ & $.31^{* *}$ & $-.27^{* *}$ & $.14^{+}$ & .08 & $.13^{+}$ & $-.16^{*}$ & $.14^{+}$ \\
Soziales Selbstkonzept (SoSK) & $.27^{*}$ & - & $.36^{* *}$ &.$- .46^{* *}$ & -.11 & -.05 & -.07 & .11 & .09 \\
Spielbezogenes Selbstkonzept (SpSK) & $.29^{*}$ & $.34^{* *}$ & - & $-.50^{* *}$ & $-.13^{+}$ & -.11 & $-.12^{+}$ & $.13^{+}$ & .06 \\
Differenziertheitsmaß (DM) & $-.19^{+}$ & $-.34^{* *}$ & $-.31^{* *}$ & - & .10 & $.16^{*}$ & $.21^{*}$ & $-.26^{* *}$ & .08 \\
Fluide Intelligenz (FI) & .13 & -.06 & -.06 & $.19^{*}$ & - & $.28^{* *}$ & $.43^{* *}$ & $-.35^{* *}$ & $.30^{* *}$ \\
Phonologisches Arbeitsgedächtnis (PA) & .08 & -.03 & .01 & .15 & $.26^{*}$ & - & $.36^{* *}$ & $-.20^{*}$ & $.27^{* *}$ \\
Exekutive Funktionen (EF) & .12 & -.14 & -.06 & $.17^{+}$ & $.50^{* *}$ & $.25^{*}$ & - & $-.47^{* *}$ & $.34^{* *}$ \\
Mathematische Vorläuferfertigkeiten (MV) & $-.22^{*}$ & .10 & .02 & -.06 & $-.36^{*}$ & -.12 & $-.47^{* *}$ & - & $-.32^{* *}$ \\
Schriftsprachliche Vorläuferfertigkeiten (SV) & $.21^{*}$ & .03 & -.14 & .04 & $.28^{*}$ & .15 & $.42^{* *}$ & $-.31^{* *}$ & - \\
\hline
\end{tabular}

Anmerkungen. Korrelationen mit paarweisem Ausschluss. Unterhalb der Diagonalen sind die Korrelationen für die Kindergartenkinder, oberhalb der Diagonalen die Korrelationen für die Schulkinder abgebildet.

${ }^{+} p<.10,{ }^{*} p<.05, * * p<.001$ 
Titel zu den Abbildungen

Abbildung 1. Vier Beispielsaufgaben der Selbstkonzeptskala, A = Übungsaufgabe,

B = Aufgabe aus der Skala des Vorakademischen Selbstkonzepts, $C=$ Aufgabe aus der Skala des Spielbezogenen Selbstkonzepts, D = Aufgabe aus der Skala des Sozialen Selbstkonzepts. 
A

die/der Größte

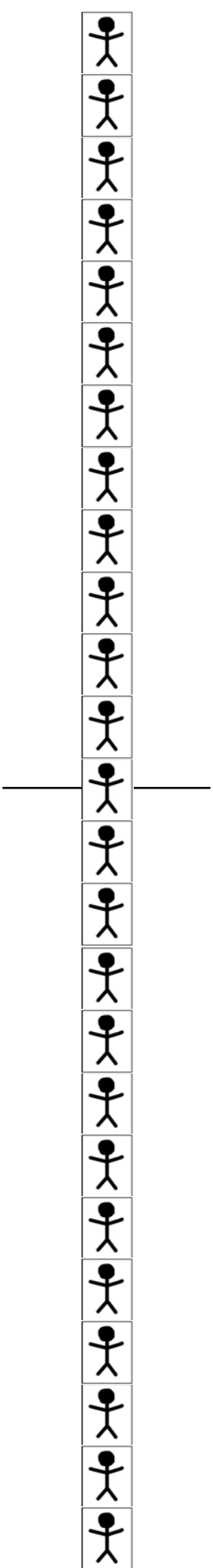

die/der Kleinste
B

die/der Beste im Buchstaben kennen

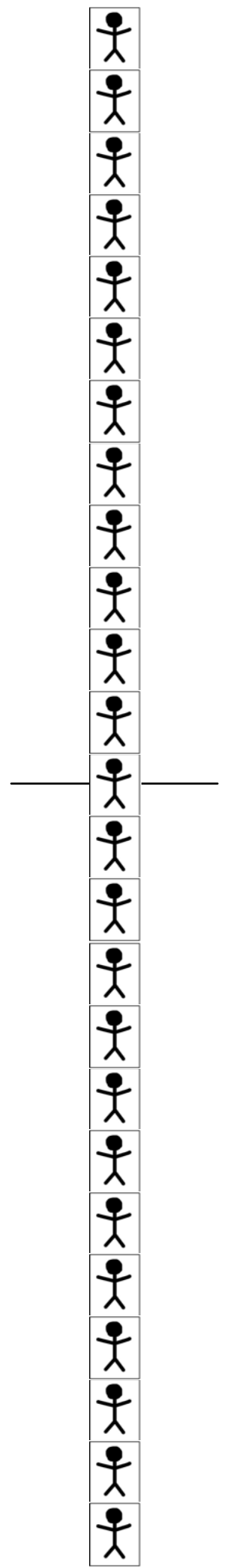

die/der Schlechteste im Buchstaben kennen
C

die/der Beste im Memory spielen

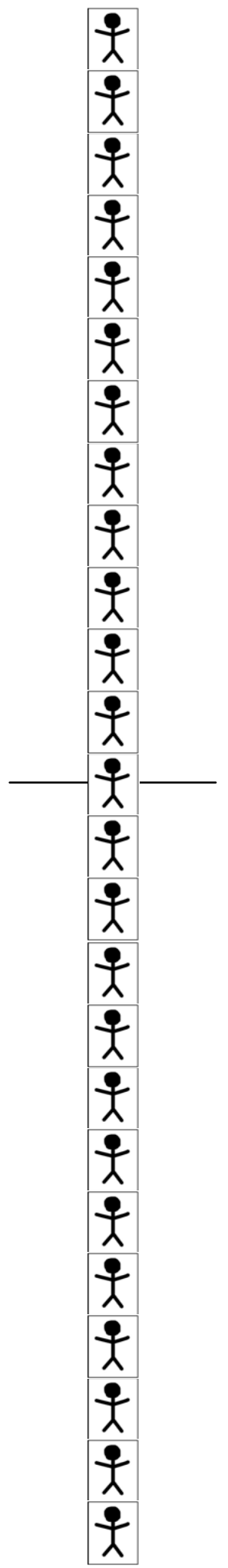

die/der Schlechteste im Memory spielen

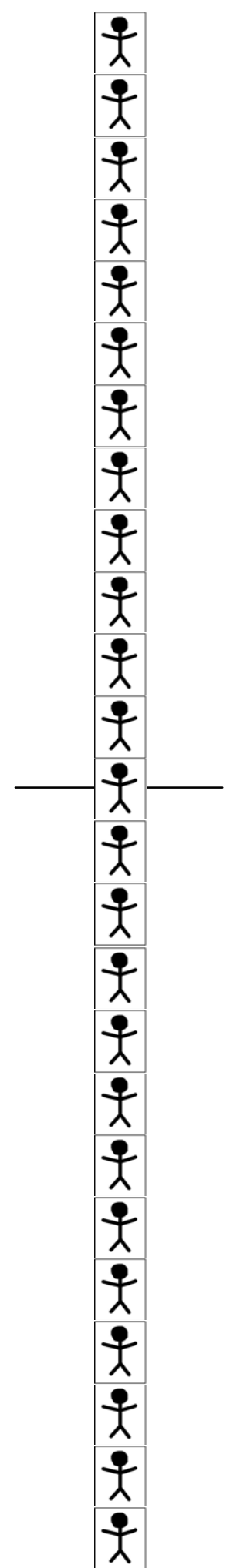

keine Freunde haben 
Studie 2: Cimeli, P., Röthlisberger, M., Neuenschwander, R., \& Roebers, C. M.

Stellt ein niedriges Selbstkonzept einen Risikofaktor für Anpassungsprobleme nach dem Schuleintritt dar? Zur Veröffentlichung eingereichtes Manuskript. 
Kolumnentitel: NIEDRIGES SELBSTKONZEPT ALS RISIKOFAKTOR

Titel: Stellt ein niedriges Selbstkonzept einen Risikofaktor für Anpassungsprobleme nach dem Schuleintritt dar?

Lic. phil. Patrizia Cimeli,

Dr. phil. Marianne Röthlisberger,

Dr. phil. Regula Neuenschwander

und

Prof. Dr. Claudia M. Roebers

Universität Bern 
Zusammenfassung Kinder im Vorschul- und frühen Grundschulalter schätzen sich sehr positiv ein. Man vermutet, dass ein hohes Selbstkonzept eine erfolgreiche Bewältigung von Entwicklungsaufgaben begünstigt. Für diese junge Altersgruppe liegen jedoch erst wenige empirische Belege dazu vor. Ziel der vorliegenden prospektiven Längsschnittstudie war es zu überprüfen, ob ein niedriges Selbstkonzept einen Risikofaktor für Anpassungsprobleme unmittelbar nach dem Schuleintritt darstellt. Dazu wurden die Entwicklungsverläufe zwischen Kindern mit niedrigem Selbstkonzept (unterstes Terzil, $n=31$ ) und Kindern mit hohem Selbstkonzept $(n=72)$ verglichen. Im Kindergarten unterschieden sich die beiden Gruppen weder in soziodemographischen noch in kognitiven Merkmalen. Nach dem Schuleintritt wiesen Kinder mit niedrigem Selbstkonzept jedoch sowohl sozial als auch leistungsmäßig eine schlechtere schulische Anpassung auf als Kinder mit hohem Selbstkonzept. Die Ergebnisse werden hinsichtlich ihrer praktischen Bedeutsamkeit diskutiert.

Schlüsselwörter: Selbstkonzept, Schuleintritt, Risikofaktor, Schulische Anpassung 
Is a low self-concept a risk factor for children's poor adjustment in elementary school?

Abstract Preschool and early elementary school children often exhibit a positive selfperception. A high self-concept is supposed to promote positive coping with developmental demands. However, empirical evidence for this young age group is scarce. The goal of the present prospective longitudinal study was to examine whether a low self-concept represents a risk factor for poor school adjustment. For this purpose, children with low self-concepts (lowest tercile, $n=31$ ) were compared to children with high self-concepts $(n=72)$. In kindergarten there were no group differences in sociodemographics or cognitive characteristics. Yet, results indicated that after school entry children with low self-concepts demonstrated lower school adjustment than children with high self-concepts, both in the social and the achievement domain. The results are discussed in terms of their practical relevance.

Key words: self-concept, school entry, risk factor, school adjustment 
Kinder im Vorschul- und frühen Grundschulalter schätzen sich selber oft sehr positiv ein, d.h. sie verfügen über ein hohes Selbstkonzept (SK; z. B. Harter, 2012). Es wird vermutet, dass diese hohe Selbsteinschätzung Teil eines normativ-adaptiven Mechanismus ist, der Kindern eine optimistische und unermüdliche Auseinandersetzung mit Entwicklungsaufgaben - wie sie typischerweise der Schuleintritt mit sich bringt erleichtert (Bjorklund \& Bering, 2002). Die entwicklungsförderliche Wirkung eines hohen bzw. SK und die entwicklungshinderliche Wirkung eines niedrigen SK konnte für Kinder ab der mittleren Grundschulzeit bereits empirisch gezeigt werden (z. B. Helmke, 1992; siehe auch Resilienzforschung von Werner, 2007). Inwiefern ein hohes bzw. niedriges SK Kindern bei den Anforderungen, die durch den Schuleintritt entstehen, entwicklungsförderlich bzw. -hinderlich wirkt, wurde bisher kaum systematisch und selten in Längsschnittstudien untersucht. Ziel der vorliegenden Studie war es deshalb, Entwicklungsverläufe zwischen Kindergartenkindern mit einem niedrigen SK und Kindergartenkindern mit einem, diesem Alter entsprechenden, hohen SK im Hinblick auf leistungsmäßige und soziale Aspekte der schulischen Anpassung zu vergleichen.

Der Schuleintritt stellt einen frühen und wichtigen Entwicklungsübergang im Leben eines Kindes dar. Das Kind sieht sich mit zentralen Entwicklungsaufgaben konfrontiert, welche neben dem Erlernen der Kulturtechniken auch ein angemessenes Leistungs- und Sozialverhalten beinhalten (z. B. Hasselhorn \& Lohaus, 2007). Die positive Bewältigung dieser alterstypischen Anforderungen gilt als Voraussetzung für eine günstige Entwicklung (Havighurst, 1972) und zeigt sich nach dem Schuleintritt in 
einer erfolgreichen schulischen Anpassung (Luthar \& Cichetti, 2000). Viele Kinder schaffen diese Anpassung problemlos, für einzelne Kinder kann der Schuleintritt jedoch zu einem kritischen Lebensereignis werden (Renner, Martschinke, Munser-Kiefer \& Steinmüller, 2011). In potentiell belastendend Entwicklungsübergängen kommen Risiko- und Schutzfaktoren besondere Bedeutung zu (Scheithauer \& Petermann, 1999). Für die entwicklungspsychologisch-klinische Forschung stellt sich deshalb die Frage, welche Faktoren auf Seite des Kindes den Übergang vom Kindergarten in die Schule begünstigen bzw. erschweren.

Neben einflussreichen familiären Hintergrundvariablen wie dem sozioökonomischen Status existiert eine Vielzahl von Merkmalen auf Seiten des Kindes, die einen wichtigen Beitrag zu einem gelungenen Start in die Schule leisten können (z. B. Petermann, 2008). So hat sich neben der fluiden Intelligenz als klassischer kognitiver Prädiktor für frühe schulische Leistungen auch die Selbstregulation (sog. Exekutive Funktionen) als entscheidend für einen erfolgreichen Übergang in die Schule erwiesen (z. B. Blair \& Razza, 2007).

Neben kognitiven und selbstregulatorischen Fähigkeiten wird auch das SK als Prädiktor für eine positive schulische Entwicklung diskutiert (z. B. Guay, Marsh \& Boivin, 2003). Das SK wird als deskriptive, kognitive Repräsentation der eigenen Fähigkeiten und Merkmale definiert (Harter, 2012).

Für Kinder im Vorschul- und frühen Grundschulalter gilt eine erhebliche Selbstüberschätzung als typisches Merkmal des SK (Nicholls, 1978). Es gibt Hinweise, dass diese überoptimistische Selbstwahrnehmung die ersten Schuljahre nach dem 
Schuleintritt zunächst anhält, bevor sie allmählich zugunsten eines realistischeren Selbstbilds zurückgeht (Cimeli, Neuenschwander, Röthlisberger \& Roebers, in press; Mantzicopoulos, 2006). Als eine wichtige Determinante dieser Selbstüberschätzung gilt das für diese Altersgruppe charakteristische, eindimensionale „Alles-oder-Nichts“-Denken (Harter, 2012). Die Selbstüberschätzung ist so eng mit den typischen kognitiven Charakteristiken der präoperationalen Phase nach Piaget (1960) verbunden und folglich als Ausdruck einer normativen Entwicklung zu verstehen. Damit drängt sich die Frage auf, inwiefern ein für dieses Alter untypisch niedriges SK entwicklungsbeeinträchtigend sein könnte.

Die theoretische Grundlage vieler empirischer SK-Studien bildet das hierarchische, multidimensionale Modell von Shavelson, Hubner und Stanton (1976), wonach das allgemeine SK in die zwei Bereiche - leistungsbezogenes, akademisches und nicht-leistungsbezogenes (d.h. soziales, emotionales und körperliches) SK unterteilt wird. Die hierarchische, ausdifferenzierte und stabile Struktur des Selbst bildet sich erst allmählich im Entwicklungsverlauf aus (z. B. Marsh \& Ayotte, 2003). Bei jungen Kindern wird das SK einerseits als relativ global und undifferenziert, gleichzeitig aber auch als situationsspezifisch und instabil beschrieben (Harter, 2012). Es gibt Hinweise, dass in diesem Alter das SK zwei unterschiedliche Dimensionen (leistungsbezogen vs. nicht-leistungsbezogen) aufweist, die jedoch nicht unabhängig voneinander sind (Harter \& Pike, 1984). Das in der vorliegenden Untersuchung eingesetzte Messinstrument beinhaltet deshalb einen leistungsbezogenen (hier: vorakademisches SK) und einen nicht-leistungsbezogenen Bereich (hier: soziales SK 
der Gleichaltrigenbeziehungen). Mit Blick auf eine erfolgreiche schulische Anpassung scheinen diese beiden Bereiche besonders wichtig zu sein (z. B. Renner et al., 2011).

Da Kinder in der frühen bis mittleren Kindheit vor allem ein situationsspezifisches SK haben, sind die dokumentierten mittleren Stabilitäten vom Kindergarten in die 1. Klasse der Grundschule erwartungsgemäss (Helmke, 1991; Measelle, Ablow, Cowan \& Cowan, 1998). Vor diesem Befundmuster scheint die Identifikation von Kindern mit einem niedrigen SK besonders entwicklungs- und gegebenenfalls auch interventionsrelevant (Verschueren, Marcoen \& Buyck, 1998).

Zusammenhänge zwischen SK und Aussenkriterien fallen bei Kindern im Kindergarten- und frühen Grundschulalter relativ gering aus. Dies gilt sowohl für Korrelationen zwischen dem leistungsbezogenen SK und Leistungsmassen wie Schulleistungstests bzw. Schulnoten $(r=.12-.20$, vgl. Metaanalyse von Hansford \& Hattie, 1982) wie auch für Korrelationen zwischen dem sozialen SK und sozialen Massen wie Einschätzungen der sozialen Kompetenz bzw. Gleichaltrigenakzeptanz (Measelle, 2005; Verschueren, Doumen \& Buyse, 2012). Insgesamt geht man heute von bidirektionalen Einflüssen zwischen SK und entsprechenden Verhaltensmassen aus (reciprocal effects model, vgl. Marsh \& Martin, 2011), wobei sich die relative Gewichtung der gegenseitigen Beeinflussung im Entwicklungsverlauf verändert. Empirische Befunde deuten darauf hin, dass der sog. self-enhancement-Ansatz spezifisch während der Phase des Schuleintritts zum Tragen kommt (Kammermeyer \& Martschinke, 2006). 
In einer Längsschnittsstudie von Verschueren, Buyck und Marcoen (2001) konnte dementsprechend gezeigt werden, dass Vorschulkinder mit einem niedrigen SK im Vergleich zu Kindern mit einem hohen SK nach dem Schuleintritt über schlechtere sozioemotionale Kompetenzen verfügten. In einer zweiten Längsschnittstudie zeigte sich ein niedriges SK als prädiktiv für schlechtere Leseleistung in den ersten drei Schuljahren (Chapman, Tunmer \& Prochnow, 2000).

Zusammengefasst liegen sowohl theoretische wie auch einige wenige empirische Hinweise vor, dass einem niedrigen SK in der Übergangsphase von 4 bis 8 Jahren eine risikoerhöhende Funktion für schulische Anpassungsprobleme zukommen kann. Unklar bleibt, inwiefern solche Effekte auch gefunden werden können, wenn schulische Anpassung als breites Konstrukt verstanden wird, das sowohl Aspekte der erbrachten Leistungen in den Kulturtechniken wie auch des konkreten Leistungs- und Sozialverhaltens beinhaltet. Dabei sollte jedoch die Vorhersagekraft wichtiger Prädiktorvariablen schulischer Anpassung gleichzeitig kontrolliert werden, um den spezifischen Einfluss des SK auf die schulische Anpassung abschätzen zu können. Vor dem Hintergrund bestehender Befunde erwarten wir deshalb bedeutsame Zusammenhänge zwischen dem SK sowie den kognitiven und soziodemographischen Variablen, welche im letzten Jahr des Kindergartens erhoben wurden und verschiedenen Indikatoren schulischer Anpassung, die in der 1. Klasse gemessen wurden. In Bezug auf die Bedeutung eines altersunüblich niedrigen SK erwarten wir, dass diese Kinder die Bewältigung der schulischen Anpassung weniger gut leisten, d.h. schlechtere Werte haben als Kindergartenkinder, die ein altersentsprechend hohes SK aufweisen. 


\section{Methode}

\section{Stichprobe}

Die Stichprobe dieser prospektiven Längsschnittstudie umfasste $N=103$ Kinder (59\% Knaben) aus der Schweiz, für die zu beiden Messzeitpunkten vollständige Daten vorlagen (ursprüngliches $N=110$, Ausfallrate: 6\%). Zum ersten Messzeitpunkt (MZP 1) befanden sich die Kinder im Kindergarten und waren $M=79.9$ Monate $(S D=3.7)$ alt. Zum zweiten Messzeitpunkt (MZP 2) besuchten alle Kinder die 1. Klasse der Grundschule (Alter: $M=92.4$ Monate, $S D=3.8$ ).

\section{Durchführung}

Zu Beginn der Längsschnittstudie wurden das Einverständnis zur Teilnahme sowie soziodemographische Angaben von Eltern und Lehrpersonen eingeholt. $\mathrm{Zu}$ MZP 1 wurden das SK der Kinder sowie kognitive Fähigkeiten (Fluide Intelligenz und Exekutive Funktionen) erfasst. Ein Jahr später (MZP 2) wurden zusätzlich zum SK verschiedene Indikatoren der schulischen Anpassung erhoben: Einschätzung des Schuleintritts, Schulleistungen, Leistungsverhalten und Sozialverhalten. Zudem wurden Informationen zu Fördermaßnahmen eingeholt. Die Rücklaufquote der Lehrpersonenund Elternfragebogen betrug $89 \%-99 \%$.

\section{Instrumente}

Der Sozioökonomische Status (SÖS) wurde anhand der Wegener Prestige-Skala (1988) erfasst; ebenso wurden Angaben zur Muttersprache des Kindes nach den Kategorien (schweizer)deutschsprachig, fremdsprachig und zweisprachig erfragt. 
Die fluide Intelligenz (FI) wurde mit dem sprachfreien Intelligenztest Test of Nonverbal Intelligence (TONI-3) von Brown, Sherbenou und Johnson (1997) erhoben.

Die Exekutiven Funktionen (EF) wurden anhand der drei Komponenten Inhibition, Updating und Shifting operationalisiert. Die abhängige Variable der EF ergab sich aus den gemittelten, z-standardisierten Variablen der Aufgaben Früchte-Gemüse-Stroop, Farbenspanne rückwärts und Kognitive Flexibilität, welche bei Röthlisberger, Neuenschwander, Michel \& Roebers (2010) beschrieben werden.

Das Selbstkonzept wurde mit der Selbstkonzeptsskala (siehe Cimeli et al., in press) erhoben. Vier Fragen bezogen sich auf den vorakademischen Bereich (Vorakademisches Selbstkonzept bzw. VaSK: „rechnen“, „zählen“, „Wörter lesen und schreiben“, „Buchstaben kennen“), vier Fragen auf den sozialen Bereich (Soziales Selbstkonzept bzw. SoSK: „viele vs. keine Freunde haben“, „,viele Kinder wollen vs. niemand will neben ihr/ihm sitzen“, „oft vs. nie zum Spielen aufgefordert werden“, „oft mit anderen Kindern zusammen vs. oft alleine spielen und lernen“). Die Kinder nahmen dabei ihre Selbsteinschätzung auf einer vertikalen Reihe von 25 geschlechtsneutralen Strichzeichnungen vor. Dem Kind wurde erklärt, dass die Figur ganz unten das Kind der Klasse repräsentiere, welches die schlechteste Leistung erbringe bzw. sich in der schwierigsten sozialen Situation befinde und die Figur ganz oben das Kind darstelle, welches die besten Leistungen erbringe bzw. sich in der besten sozialen Situation befinde. Als abhängige Variable diente die vom Kind markierte Position auf der vertikalen Reihe (1-25). Die zwei Skalen VaSK $(\alpha=.72)$ und SoSK $(\alpha=.60)$ korrelierten untereinander zu $r=.28, p<.01$. Die Reliabilität der Gesamtskala aller acht 
Fragen, das generelle SK (GSK), beträgt $\alpha=.70$. Kinder, welche sich im untersten Drittel der GSK-Verteilung befanden, wurden in die Gruppe der Kinder mit einem niedrigen SK ( $\mathrm{SK}_{\text {niedrig }}$ ) eingeteilt $(n=31, M=13.8, S D=1.6)$. Die restliche Stichprobe wurde als Gruppe der Kinder mit einem „,normalen“ SK (SK norm$)$ definiert $(n=72$, $M=21.1, S D=3.0)$.

\section{Schulische Anpassung}

Schuleintritt. Lehrpersonen und Eltern schätzten die Aussage „Das Kind bzw. Mein Kind hat den Schuleintritt gut gemeistert“ auf einer Skala von 1 (stimmt gar nicht) bis 4 (stimmt genau) ein.

Schulleistungen. Die Leistungen in den Fächern Mathematik und Schriftsprache wurden über standardisierte Schulleistungstests und über Beurteilungen der Lehrpersonen erhoben. Zur Erfassung der mathematischen Leistungen wurden zwei Untertests Größenvergleich und Rechenoperationen aus dem HRT 1-4 von Haffner, Baro, Parzer und Resch (2005) adaptiert und eingesetzt. Die schriftsprachlichen Leistungen wurden anhand der Lese- und die Rechtschreibkompetenzen der Kinder ermittelt. Die Lesekompetenz wurde mit der WLLP von Küspert und Schneider (1998), die Rechtschreibkompetenz mit einer adaptierten Version der HSP von May (2002) erfasst. Die z-standardisierten, gemittelten Werte der entsprechenden Untertests ergaben die abhängige Variable der Schulleistungen Mathematik bzw. Schriftsprache. Die Lehrpersonen wurden gebeten, die Schulleistungen der Kinder in den Fächern Mathematik, Lesen und Schreiben auf einer Skala 1 (ungenügend) bis 4 (sehr gut) zu beurteilen. Die Beurteilung in Mathematik diente direkt als abhängige Variable, die 
Beurteilungen in Lesen und Schreiben wurden zur abhängigen Variablen der Beurteilung in Schriftsprache gemittelt.

Leistungsverhalten. Zur Erfassung des globalen Leistungsverhaltens wurden den Lehrpersonen $(\alpha=.80)$ und den Eltern $(\alpha=.79)$ je fünf Fragen zur Lernfreude gestellt. Zwei Fragen waren der IDS von Grob, Meyer und Hagmann-Von Arx (2009) entnommen und für Lehrpersonen und Eltern identisch. Das spezifische Leistungsverhalten wurde bei Lehrperson und Eltern anhand derselben zehn Fragen zum Durchhaltevermögen erhoben, vier davon entstammten der IDS. Während sich die Fragen der Lehrpersonen auf das kindliche Verhalten in einer Stillarbeitssituation bezogen $(\alpha=.95)$, wurde bei den Eltern auf das kindliche Verhalten während der Erledigung der Hausaufgaben fokussiert $(\alpha=.85)$.

Sozialverhalten. Die soziale Anpassung des Kindes an den Klassenverband wurde bei den Lehrpersonen mit drei Fragen auf einer 4-Punkte-Skala erhoben $(\alpha=.89)$.

Fördermaßnahmen. Die Lehrpersonen gaben Auskunft darüber, ob ein Kind den Status eines/r Regelschülers/in oder eines/r Schülers/in mit speziellem Förderbedarf innehatte. Von den 103 Kindern wurden 14 Kinder als Schüler/innen mit speziellem Förderbedarf eingestuft. Bei den Eltern wurde zudem erfragt, ob ihr Kind (unabhängig vom Status) zusätzlichen Förderunterricht erhielt.

\section{Ergebnisse}

Tabelle 1 zeigt die Korrelationen zwischen den Variablen, die zu MZP 1 im Kindergarten erfasst wurden und den Indikatoren schulischer Anpassung von MZP 2. 
Zwischen dem GSK und der Mehrzahl der Indikatoren schulischer Anpassung ergaben sich relativ geringe, aber dennoch bedeutsame Korrelationen. Für die Unterskalen VaSK und SoSK zeigte sich dabei ein bereichsspezifisches Prädiktionsmuster. Die Korrelationen zwischen Geschlecht und Indikatoren schulischer Anpassung wiesen außer in den Schulleistungen Mathematik auf bessere Werte der Mädchen im Vergleich zu den Jungen hin. Während der SÖS lediglich für die Testleistungen in Mathematik prädiktiv war, fanden sich zwischen FI bzw. EF und Indikatoren schulischer Anpassung mit Ausnahme der sozialen Anpassung mittlere Zusammenhänge.

- hier Tabelle 1 einfügen -

Die beiden SK-Gruppen wurden in einem ersten Analyseschritt bezüglich soziodemographischer und kognitiver Variablen miteinander verglichen. Die Ergebnisse in Tabelle 2 zeigen, dass sich die beiden Gruppen in keinem der einbezogenen Merkmale bedeutsam voneinander unterschieden. Auch was die Muttersprache betraf, unterschied sich die Häufigkeitsverteilung der drei Kategorien nicht bedeutsam zwischen den beiden Gruppen, $\chi^{2}(2)=2.57$, n.s.

$$
\text { - hier Tabelle } 2 \text { einfügen - }
$$

Um Aussagen über Unterschiede im Entwicklungsverlauf der beiden SK-Gruppen nach dem Schuleintritt machen zu können, wurden die zwei SK-Gruppen hinsichtlich schulischer Anpassung miteinander verglichen. Tabelle 3 zeigt, dass

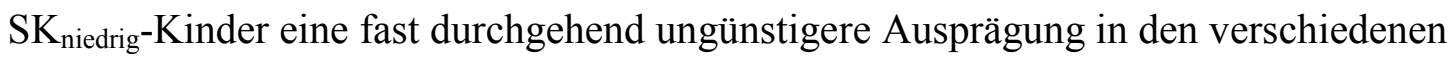
Indikatoren erfolgreicher schulischer Anpassung zeigten als $\mathrm{SK}_{\text {norm }}-$ Kinder. Selbst wenn soziodemographische und kognitive Merkmale von MZP 1 als Kovariaten in die 
Varianzanalyse einbezogen wurden, blieb dieses Befundmuster zugunsten der $\mathrm{SK}_{\text {norm}}$-Kinder weitgehend bestehen.

$$
\text { - hier Tabelle } 3 \text { einfügen - }
$$

Um zu überprüfen, wie stabil sich das SK der Kinder vom Kindergarten in die 1. Klasse verhält, wurde mithilfe einer Varianzanalyse mit Messwiederholung überprüft, wie sich das SK innerhalb der beiden Gruppen über die Zeit veränderte. Es ergaben sich ein signifikanter Haupteffekt SK-Gruppe $F(1,101)=98.49, p<.001, \eta^{2}=.49$, und ein signifikanter Haupteffekt Zeit $F(1,101)=21.60, p<.001, \eta^{2}=.18$. Obwohl die signifikante Interaktion MZP $\times$ Gruppe, $F(1,101)=24.16, p<.001, \eta^{2}=.19$, auf eine gruppenspezifische Veränderung des SK im Entwicklungsverlauf hindeutete, ergab eine einfaktorielle Varianzanalyse für MZP 2, dass auch nach Schuleintritt das SK bei den $\mathrm{SK}_{\text {niedrig}}$-Kindern immer noch signifikant niedriger ausfiel als bei den $\mathrm{SK}_{\text {norm}}$-Kindern $(M=21.0, S D=3.4), F(1,101)=13.13, p<.001, \eta^{2}=.12$. Die Korrelation zwischen dem GSK von MZP 1 und dem GSK von MZP 2 betrug $r=.34, p<.001$. Von den ursprünglichen $\mathrm{SK}_{\text {niedrig }}$-Kindern wurden $42 \%$ ein Jahr später noch immer $\mathrm{SK}_{\text {niedrig}}$-Kinder kategorisiert, 58\% hingegen waren zu SK $\mathrm{norm}$-Kindern ,aufgestiegen“.

Es wurde abschließend überprüft, ob sich $\mathrm{SK}_{\text {niedrig }}$-Kinder von $\mathrm{SK}_{\text {norm}}$-Kinder hinsichtlich eingeleiteter Fördermaßnahmen unterschieden. Unter den 14 Kindern der gesamten Stichprobe, welche mit einem Status als Schüler/innen mit speziellem Förderbedarf eingeschult wurden, befanden sich anteilsmäßig mehr $\mathrm{SK}_{\text {niedrig-Kinder als }}$ $\mathrm{SK}_{\text {norm }}$-Kinder, $\chi^{2}(1)=3.05, p<.10$. Was den Förderunterricht betraf, so wurden 
tendenziell mehr $\mathrm{SK}_{\text {niedrig }}$-Kinder (45\%) gefördert als dies bei den $\mathrm{SK}_{\text {norm }}$-Kindern $(26 \%)$ der Fall war, $\chi^{2}(1)=3.32, p<.10$.

\section{Diskussion}

Das Ziel der vorliegenden prospektiven Längsschnittstudie war es, zu überprüfen, inwiefern ein altersuntypisch niedriges SK von Kindergartenkindern einen Risikofaktor für schulische Anpassungsprobleme in der 1. Klasse darstellt. Dazu wurden zwei Gruppen von Kindern verglichen, die vor dem Schuleintritt entweder ein niedriges SK oder ein, diesem Alter entsprechendes, hohes SK aufwiesen. Die zwei Gruppen unterschieden sich im Kindergarten weder in kognitiven noch in soziodemographischen Merkmalen. Im Entwicklungsverlauf ergaben sich jedoch nach dem Schuleintritt hinsichtlich der schulischen Anpassung signifikante Unterschiede. Kinder, welche am Ende des Kindergartens ein niedriges SK hatten, wiesen in der 1. Klasse sowohl leistungsmässig wie auch sozial schlechtere schulische Anpassungswerte auf als Kinder, welche ein alterstypisch ausgeprägtes SK hatten.

Die vorliegenden Ergebnisse lassen somit vermuten, dass ein niedriges SK tatsächlich als Risikofaktor für Anpassungsprobleme nach dem Schuleintritt bezeichnet werden kann. Zu dieser Schlussfolgerung führt insbesondere die Tatsache, dass sich die Kinder zu MZP 1 im Kindergarten einzig in der Ausprägung des SK unterschieden (vgl. Tabelle 2), nicht aber in den Ausgangsvariablen Geschlecht, Alter, SÖS, FI und EF, welche sich zudem wie erwartet als weitgehend bedeutsame Prädiktoren schulischer Anpassung erwiesen (vgl. Tabelle 1). Die unterschiedlichen Entwicklungsverläufe in der schulischen Anpassung (vgl. Tabelle 3) können aufgrund der uns zur Verfügung 
stehenden Daten also einzig auf die Zugehörigkeit zu den Gruppen $\mathrm{SK}_{\text {niedrig }}$ bzw. $\mathrm{SK}_{\text {norm }}$ zurückgeführt werden. Wenn das SK hier im Gruppenvergleich einen spezifischen Beitrag zur Erklärung früher schulischer Anpassung leisten kann, deutet dies auf eine wichtige Funktion des SK in Übergängen, wie hier vom Kindergarten in die Schule, hin (vgl. Filipp, 2006).

Offen bleibt, ob ein niedriges SK auch einen praktisch relevanten Risikofaktor darstellt; inwiefern also die Unterschiede in Bezug auf die schulische Anpassungsleistung auch tatsächlich praktisch bedeutsam sind. Einerseits sprechen die kleinen Effekte, als welche die gefundenen Unterschiede in der schulischen Anpassung nach Cohen (1969) kategorisiert werden, eher für eine geringe praktische Bedeutung. Andererseits ergeben sich in unseren Daten durchaus Hinweise auf die praktische Relevanz der Effekte. So wurden $\mathrm{SK}_{\text {niedrig}}$-Kinder in ihrer schlechteren schulischen Anpassung durch unterschiedliche Quellen (objektive Tests, Lehrpersonen und Eltern) fast übereinstimmend erkannt, sie zeigten sich in unterschiedlichen Anpassungsbereichen (leistungsmäßig und sozial) auffällig und sie erhielten zudem tendenziell häufiger spezielle Fördermaßnahmen als $\mathrm{SK}_{\text {norm }}$-Kinder. Die vorliegenden Befunde konnten somit unserer Meinung nach zeigen, dass auch sog. kleine Effekte eine praktische Bedeutung im (schulischen) Alltag haben können.

Weitere Hinweise auf die praktische Bedeutsamkeit der Effekte liefern die gefundenen Stabilitätswerte des SK von mittlerer Höhe, wie sie ähnlich schon in anderen Studien gefunden wurden (z. B. Measelle et al., 1998). Eine mittlere Stabilität weist darauf hin, dass zwar die Möglichkeit zur Veränderungen im SK gegeben ist, dass 
aber das SK keineswegs als ausschließlich situationsabhängig bezeichnet werden kann (vgl. Harter, 2012). Zusätzlich muss beachtet werden, dass diese mittleren Stabilitäten trotz eines Bezugsgruppenwechsels vom Kindergarten in die Schule und trotz eines SK-Messinstruments zustande kamen, welches gerade auf dem sozialen Vergleich mit der aktuellen Bezugsgruppe basiert (Gabriel, Kastens, Poloczek, Schoreit \& Lipowsky, 2010). Betrachtet man nur die $\mathrm{SK}_{\text {niedrig}}-$ Kinder, so besteht für beinahe die Hälfte dieser Kinder eine gewisse Gefahr der „Chronifizierung“ eines schlechten Selbstbilds im weiteren Entwicklungsverlauf. Im Sinne der praktischen Bedeutsamkeit handelt es sich möglicherweise bei dieser Subgruppe um Kinder, welche in dieser Übergangsituation konkret einer Intervention bedürfen.

Ziele zukünftiger Forschung sollten darin bestehen, die praktische Bedeutsamkeit der hier dokumentierten Effekte zu verifizieren und Faktoren zu ermitteln, welche die Entstehung eines niedrigen SK begünstigen. Auch mögliche direkte und indirekte Mechanismen zwischen dem SK und Entwicklungsergebnissen sollten weiterführend untersucht werden. Interessant wäre auch zu beobachten, wie sich Kinder mit niedrigem SK längerfristig entwickeln.

Insgesamt erbrachte diese für den deutschsprachigen Bildungsraum erste prospektive Längsschnittstudie Hinweise darauf, dass das SK einen wichtigen Beitrag zu einem gelungenen Start in die Schule leisten kann. 
Bjorklund, D. F. \& Bering, J. M. (2002). The evolved child: Applying evolutionary developmental psychology to modern schooling. Learning and Individual Differences, 12, 347-373.

Blair, C. \& Razza, R. P. (2007). Relating effortful control, executive function, and false belief understanding to emerging math and literacy ability in kindergarten. Child Development, 78, 647-663.

Brown, L., Sherbenou, R. J. \& Johnson, S. K. (1997). TONI-3: Test of nonverbal intelligence: A language-free measure of cognitive ability (3rd ed.). Austin: ProEd.

Chapman, J. W., Tunmer, W. E. \& Prochnow, J. E. (2000). Early reading-related skills and performance, reading self-concept, and the development of academic selfconcept: A longitudinal study. Journal of Educational Psychology, 92, 703-708.

Cimeli, P., Neuenschwander, R., Röthlisberger, M. \& Roebers, C. M. (in press). Das Selbstkonzept von Kindern in der Schuleingangsphase: Ausprägung und Struktur sowie Zusammenhänge mit frühen kognitiven Leistungsindikatoren. Zeitschrift für Entwicklungspsychologie und Pädagogische Psychologie.

Cohen, J. (1969). Statistical power analysis for the behavioral sciences. New York, London: Academic Press.

Filipp, S. H. (2006). Kommentar zum Themenschwerpunkt. Entwicklung von Fähigkeitsselbstkonzepten. Zeitschrift für Entwicklungspsychologie und Pädagogische Psychologie, 20, 65-72. 
Gabriel, K., Kastens, C., Poloczek, S., Schoreit, E. \& Lipowsky, F. (2010). Entwicklung des mathematischen Selbstkonzepts im Anfangsunterricht - Der Einfluss des Klassenkontextes. Zeitschrift für Grundschulforschung, 3, 65-82.

Grob, A., Meyer, S. C. \& Hagmann-von Arx, P. (2009). Intelligence and Development Scales (IDS). Bern: Verlag Hans Huber.

Guay, F., Marsh, H. W. \& Boivin, M. (2003). Academic self-concept and academic achievement: Developmental perspectives on their causal ordering. Journal of Educational Psychology, 95, 124-136.

Haffner, J., Baro, K., Parzer, P. \& Resch, F. (2005). Heidelberger RechentestHRT 1-4: Erfassung mathematischer Basiskompetenzen im Grundschulalter. Göttingen: Hogrefe.

Hansford, B. C. \& Hattie, J. A. (1982). The relationsship between self and achievement/performance measures. Review of Educational Research, 52, 123142.

Harter, S. (2012). The construction of the self: A developmental perspective (Vol. 2). New York: Guilford Press.

Harter, S. \& Pike, R. (1984). The pictorial scale of perceived competence and social acceptance for young children. Child Development, 55, 1969-1982.

Hasselhorn, M. \& Lohaus, A. (2007). Schuleintritt. In M. Hasselhorn \& W. Schneider (Hrsg.), Handbuch der Entwicklungspsychologie. Göttingen: Hogrefe.

Havighurst, R. J. (1972). Developmental tasks and education. New York: McKay. 
Helmke, A. (1991). Entwicklung des Fähigkeitsselbstbildes vom Kindergarten bis zur dritten Klasse. In R. Pekrun \& H. Fend (Hrsg.), Schule und Persönlichkeitsentwicklung - ein Resümee der Längsschnittforschung. Stuttgart: Ferdinand Enke Verlag.

Helmke, A. (1992). Selbstvertrauen und schulische Leistungen. Göttingen: Hogrefe.

Kammermeyer, G. \& Martschinke, S. (2006). Selbstkonzept- und Leistungsentwicklung in der Grundschule - Ergebnisse aus der KILIA-Studie. Empirische Pädagogik, $20,245-259$.

Küspert, P. \& Schneider, W. (1998). Würzburger Leise Leseprobe (WLLP). Göttingen: Hogrefe.

Luthar, S., S. \& Cichetti, D. (2000). The construct of resilience: Implications for interventions and social policies. Developmental Psychopathology, 12, 857-885.

Mantzicopoulos, P. (2006). Younger children`s changing self-concept: Boys and girls from preschool through second grade. The Journal of Genetic Psychology, 167, 289-308.

Marsh, H. W. \& Ayotte, V. (2003). Do multiple dimensions of self-concept become more differentiated with age? The differential distinctiveness hypothesis. Journal of Educational Psychology, 95, 687-706.

Marsh, H. W. \& Martin, A. J. (2011). Academic self-concept and academic achievement: Relations and causal ordering. British Journal of Educational Psychology, 81, 59-77.

May, P. (2002). Hamburger Schreib-Probe (HSP) 1-9 (6. Aufl.). Hamburg: vpm. 
Measelle, J., R. (2005). Children`s self-perceptions as a link between familiy relationship quality and social adaptation to school. In P. Cowan, A., C. Cowan, P., J. Ablow, C., V. K. Johnson \& J. Measelle, R. (Eds.), The family context of parenting in children `s adaptation to elementary school. New Jersey: Lawrence Erlbaum Associates.

Measelle, J., R., Ablow, J., C., Cowan, P., A. \& Cowan, C., P. (1998). Assessing young childrens`s view of their academic, social, and emotional lives: An evaluation of the self-perception scales of the Berkeley Puppet Interview. Child Development, $69,1556-1576$.

Nicholls, J. G. (1978). The development of the concepts of effort and ability, perception of academic attainment, and the understanding that difficult tasks require more ability. Child Development, 49, 800-814.

Petermann, F. (2008). Editorial zum Themenschwerpunkt: Kompetenz- und Leistungsdiagnostik zum Schuleintritt. Psychologie in Erziehung und Unterricht - Zeitschrift für Forschung und Praxis, 55, 81-83.

Piaget, J. (1960). The psychology of intelligence. Patterson: Littlefield-Adams.

Renner, G., Martschinke, S., Munser-Kiefer, M. \& Steinmüller, S. (2011). Diagnose und Förderung des Selbstkonzepts im Anfangsunterricht. In F. Hellmich (Hrsg.), Selbstkonzepte im Grundschulalter: Modelle, empirische Ergebnisse, pädagogische Konsequenzen. Stuttgart: Kohlhammer.

Röthlisberger, M., Neuenschwander, R., Michel, E. \& Roebers, C. M. (2010). Exekutive Funktionen: Zugrundeliegende kognitive Prozesse und deren Korrelate bei 
Kindern im späten Vorschulalter. Zeitschrift für Entwicklungspsychologie und Pädagogische Psychologie, 42, 99-110.

Scheithauer, H. \& Petermann, F. (1999). Zur Wirkungsweise von Risiko- und Schutzfaktoren in der Entwicklung von Kindern und Jugendlichen. Kindheit und Entwicklung, 8, 3-14.

Shavelson, R. J., Hubner, J. J. \& Stanton, G. C. (1976). Self-concept: Validation of construct interpretation. Review of Educational Research, 46, 407-441.

Verschueren, K., Buyck, P. \& Marcoen, A. (2001). Self-representations and socioemotional competences in young children: A 3-year longitudinal study. Developmental Psychology, 37, 126-134.

Verschueren, K., Doumen, S. \& Buyse, E. (2012). Relationships with mother, teacher, and peers: Unique and joint effects on young children`s self-concept. Attachment \& Human Development, 14, 233-248.

Verschueren, K., Marcoen, A. \& Buyck, P. (1998). Five-year-old`s behaviorally presented self-esteem: Relations to self-perceptions and stability across a threeyear period. The Journal of Genetic Psychology, 159, 273-279.

Wegener, B. (1988). Kritik des Prestiges: Opladen: Westdeutscher Verlag.

Werner, E. E. (2007). Resilienz: Ein Überblick über internationale Längsschnittstudien. In G. Opp \& M. Fingerle (Hrsg.), Was Kinder stärkt - Erziehung zwischen Risiko und Resilienz. München: Ernst Reinhardt Verlag. 
Autorenadresse:

Lic. phil. Patrizia Cimeli, patrizia.cimeli@psy.unibe.ch (Korrespondenzadresse)

Dr. phil. Regula Neuenschwander

Dr. phil. Marianne Röthlisberger

Prof. Dr. Claudia M. Roebers

Universität Bern, Institut für Psychologie

Abteilung Entwicklungspsychologie, Muesmattstraße 45, CH-3000 Bern, 
Tabelle 1. Korrelationen zwischen den kindlichen Variablen zu Messzeitpunkt 1 (Kindergarten) und den Indikatoren früher schulischer Anpassung zu Messzeitpunkt 2 (1. Klasse), $N_{\text {Total }}=103$

\begin{tabular}{|c|c|c|c|c|c|c|}
\hline & $\begin{array}{l}\text { Schuleintritt } \\
\text { (LP/EL) }\end{array}$ & $\begin{array}{l}\text { Schulleistungen } \\
\text { Mathematik } \\
\text { (LP/Test) }\end{array}$ & $\begin{array}{l}\text { Schulleistungen } \\
\text { Schriftsprache } \\
\text { (LP/Test) }\end{array}$ & $\begin{array}{l}\text { Leistungsverhalten } \\
\text { global: Lernfreude } \\
\text { (LP/EL) }\end{array}$ & $\begin{array}{l}\text { Leistungsverhalten } \\
\text { spezifisch: } \\
\text { Durchhaltevermögen } \\
\text { (LP/EL) }\end{array}$ & $\begin{array}{l}\text { Sozialverhalten: } \\
\text { Soziale } \\
\text { Anpassung } \\
\text { (LP) }\end{array}$ \\
\hline Generelles SK & $.17^{+} / .23^{*}$ & $.10 / .23 *$ & $.16 / .19^{+}$ & $.21 * / .17^{+}$ & $.21 * / .15$ & .13 \\
\hline Vorakademisches SK & $.19^{+} / .18^{+}$ & $.17 / .25^{*}$ & $.28 * / .27 *$ & $.22 * / .17^{+}$ & $.22 * / .13$ & .03 \\
\hline Soziales SK & $.07 / .20 *$ & $-.03 / .10$ & $-.08 /-.02$ & $.09 / .09$ & $.10 / .11$ & $.21^{*}$ \\
\hline Geschlecht & $-.31 * /-.25 *$ & $.01 / .05$ & $-.31 * /-.22 *$ & $-.32 * /-.21 *$ & $-.34 * * / .-.30 *$ & $-.19^{+}$ \\
\hline Alter in Monaten & $.13 / .26^{*}$ & $.17^{+} / .13$ & $.16 / .22 *$ & $.25 * / .10$ & $.29 * / .04$ & $.24 *$ \\
\hline Sozioökonomischer Status & $-.06 /-.01$ & $.17 / .26^{*}$ & $-.08 / .13$ & $.08 /-.11$ & $-.08 /-.13$ & -.09 \\
\hline Fluide Intelligenz & $.32 * / .14$ & $.40 * * / .32 *$ & $.31 * / .39 * *$ & $.29 * / .21 *$ & $.19^{+} / .20^{*}$ & .08 \\
\hline Exekutive Funktionen & $.32 * / .33 *$ & $.43 * * / .50 * *$ & $.35 * * / .53 * *$ & $.43 * * / .31 *$ & $.49 * * / .25 *$ & .16 \\
\hline
\end{tabular}

Anmerkungen. SK: Selbstkonzept, LP: Einschätzung Lehrperson, EL: Einschätzung Eltern, Geschlecht: Negative Korrelationen bedeuten höhere Werte bei den Mädchen als bei den Jungen.

${ }^{+} p<.10,{ }^{*} p<.05, * * p<.001$ 
Tabelle 2. Vergleich der soziodemographischen und kognitiven Merkmale zwischen Kindern mit niedrigem Selbstkonzept (SK niedrig$)$ und Kindern mit altersentsprechend ausgeprägtem Selbstkonzept $\left(\mathrm{SK}_{\text {norm }}\right)$ zu Messzeitpunkt 1 (Kindergarten), $N_{\text {total }}=103$

\begin{tabular}{lccc}
\hline & SK $_{\text {niedrig }}$ & SK norm & $\begin{array}{c}\text { Signifikanztest } \\
\left(F-\text { bzw. } \chi^{2}-\mathrm{Wert}\right)\end{array}$ \\
\hline$N$ & 31 & 72 & - \\
Geschlecht (männlich, \%) & $71 \%$ & $54 \%$ & $\chi^{2}(1)=2.53$, n.s. \\
Alter in Monaten & $79.5(3.6)$ & $80.1(3.7)$ & $F(1,103)=0.63, n . s$. \\
Sozioökonomischer Status & $8.6(3.7)$ & $8.9(3.3)$ & $F(1,91)=0.08, n . s$. \\
Fluide Intelligenz & $97.2(8.0)$ & $98.2(10.6)$ & $F(1,102)=0.24$, n.s. \\
Exekutive Funktionen & $-0.11(0.6)$ & $0.05(0.8)$ & $F(1,73.8)^{\mathrm{a}}=1.24, n . s$. \\
\hline
\end{tabular}

Anmerkungen. Sozioökonomischer Status: Werte gemäß Wegener-Prestige Skala d.h. Aufsummierung der Prestigewerte beider elterlicher Ausbildungen und des Prestigewerts der Berufsausübung mit höherer Einstufung, Fluide Intelligenz: IQ-Wert, Exekutiven Funktionen: Mittelwert der z-standardisierten Variablen dreier Aufgaben, ${ }^{a}=F$-Wert nach Welch inkl. angepasster Freiheitsgrade. 
Tabelle 3. Vergleich verschiedener Indikatoren schulischer Anpassung zwischen Kindern mit niedrigem Selbstkonzept $\left(\mathrm{SK}_{\text {niedrig}}\right)$ und Kindern mit altersentsprechend ausgeprägtem Selbstkonzept ( $\mathrm{SK}_{\text {norm }}$ zu Messzeitpunkt 2 (1. Klasse), $N_{\text {total }}=103$

\begin{tabular}{ccc}
\hline $\mathrm{SK}_{\text {niedrig }}$ & $\mathrm{SK}_{\text {norm }}$ & Signifikanztest \\
$(n=31)$ & $(n=72)$ & $(F$-Wert $)$ \\
\hline
\end{tabular}

Schuleintritt

$\begin{array}{cccc}\text { Einschätzung LP } & 3.2(0.9) & 3.6(0.7) & F(1,45.5)^{\mathrm{a}}=3.46, p<.10, \eta_{\mathrm{p}}{ }^{2}=.04 \\ \text { Einschätzung EL* } & 3.5(0.6) & 3.8(0.5) & F(1,42.4)^{\mathrm{a}}=6.61, p<.05, \eta_{\mathrm{p}}{ }^{2}=.08\end{array}$

Schulleistungen

$\begin{array}{rccc}\text { Mathematik (LP) } & 3.0(0.8) & 3.1(0.8) & F(1,99)=0.70, \text { n.s. } \\ \text { Mathematik (Test)* } & -0.3(0.7) & 0.1(0.9) & F(1,101)=4.04, p<.05, \eta_{\mathrm{p}}{ }^{2}=.04 \\ \text { Schriftsprache (LP) } & 2.8(0.8) & 3.1(0.7) & F(1,101)=3.64, p<.10, \eta_{\mathrm{p}}{ }^{2}=.04 \\ \text { Schriftsprache (Test)* } & -0.3(0.8) & 0.1(0.9) & F(1,101)=4.28, p<.05, \eta_{\mathrm{p}}{ }^{2}=.04\end{array}$

Leistungsverhalten

(global und spezifisch)

$\begin{array}{rrrr}\text { Lernfreude (LP) } & 3.2(0.6) & 3.4(0.5) & F(1,101)=2.80, p<.10, \eta_{\mathrm{p}}{ }^{2}=.03 \\ \text { Lernfreude (EL)* } & 3.3(0.4) & 3.5(0.5) & F(1,99)=4.10, p<.05, \eta_{\mathrm{p}}{ }^{2}=.04 \\ \text { Durchhaltevermögen (LP)** } & 2.6(0.8) & 3.1(0.8) & F(1,101)=7.26, p<.01, \eta_{\mathrm{p}}{ }^{2}=.07 \\ \text { Durchhaltevermögen (EL)** } & 2.7(0.4) & 3.0(0.5) & F(1,99)=7.30, p<.01, \eta_{\mathrm{p}}{ }^{2}=.07\end{array}$

Sozialverhalten

$$
\text { Soziale Anpassung (LP)* } 3.1(0.8) \quad 3.5(0.7) \quad F(1,101)=5.88, p<.05, \eta_{\mathrm{p}}{ }^{2}=.06
$$

Anmerkungen. ${ }^{\mathrm{a}}=F$-Wert nach Welch inkl. angepasste Freiheitsgrade. $\eta_{\mathrm{p}}{ }^{2}=$ partielles

Eta-Quadrat (Effektstärke), LP: Einschätzung Lehrperson, EL: Einschätzung Eltern.

${ }^{+} p<.10, * p<.05, * * p<.01$ 
Studie 3: Roebers, C. M., Röthlisberger, M., Neuenschwander, R., \& Cimeli, P.

School progress, academic performance, and self-regulatory processes as a function of early elementary school context. [Schulischer Werdegang, schulische Leistungen und selbst-regulatorische Prozesse in Abhängigkeit des Schuleingangsmodells] Manuscript resubmitted for publication. 
School progress, academic performance, and self-regulatory processes as a function of early elementary school context

Schulischer Werdegang, schulische Leistungen, und selbst-regulatorische Prozesse in Abhängigkeit des Schuleingangsmodells

Claudia M. Roebers, Marianne Röthlisberger,

Regula Neuenschwander, and Patrizia Cimeli

Center of Cognition, Learning, and Memory

University of Bern, Switzerland 
Zusammenfassung

In der vorliegenden Studie wurden Schulleistungen, Selbstkonzept, Exekutive Funktionen und metakognitive Fähigkeiten in Abhängigkeit der Zugehörigkeit zu zwei

Schuleingangsmodellen untersucht. Eine Stichprobe mit $N=230$ Kindern wurde untersucht, von denen $50 \%$ der 7- bis 8- jährigen Kinder das traditionelle Schuleingangsmodell besuchten (1. oder 2. Klassenstufe), und $50 \%$ der Kinder sich in der sogenannten "Basisstufe" befanden, einem jahrgangsübergreifenden Schuleingangsmodell, welches individualisierte Lernformen beinhaltet. Obwohl im traditionellen Schuleingangsmodell mehr Kinder in Spezialklassen ausgesondert wurden oder ein Schuljahr wiederholen mussten als in der Basisstufe, zeigten die Kinder in der Basisstufe vergleichbare Schulleistungen wie die Kinder im traditionellen Schuleingangsmodell. Auch in den anderen, einbezogenen Indikatoren, die mit selbst-regulierten Prozessen in Verbindung gebracht werden, konnten kaum praktisch bedeutsame Unterschiede zwischen den beiden Schuleingangsmodellen gefunden werden. Die Ergebnisse werden zum einen vor dem Hintergrund der aktuellen schulpolitischen Debatte zum Schulübertritt und zur frühen Bildung diskutiert. Zum anderen geben die vorliegenden Befunde Hinweise auf die Veränderbarkeit von wichtigen Schülermerkmalen durch schulische Einflüsse.

Schlüsselwörter: Basisstufe, Schuleintritt, Metakognitive Fähigkeiten, Exekutive Funktionen, Vorakademisches Selbstkonzept, Schulleistungen 
Abstract

Against the background of an integrative theoretical framework of self-regulated learning, a broad range of students` characteristics, including academic achievement, self-concept, executive functioning, and metacognitive processes were examined as a function of children`s early school context. A sample of $N=230$ children was included in the study. While half of the participating 7- to 8-yr-olds attended classes in the traditional school system, the other half of the sample was enrolled in classes that embraced kindergarten to $2^{\text {nd }}$ grade students in mixed-grades classes (Basisstufe) and that emphasized individualized learning opportunities and flexible grade progression. Although in the traditional classes more children had been segregated or repeated a grade than in the heterogeneous mixed-grades classes, academic performance in the mixed-grades classes were comparable. Results are discussed against the background of issues concerning early school transition and early education as well as in terms of environmental factors (school contexts) theoretically influencing central students` characteristics.

Keywords:

Mixed-grades classes, school transition, metacognitive processes, executive functions, preacademic self-concept, academic performance 


\section{Introduction}

Pre-primary and primary education has received increased attention from two sides: on the one side, social policy makers in many different countries have recognized the importance of uniform access to high quality early education for young children for facilitating the transition into primary education and for reaching and fostering children in need of special support; for example, children with special needs, from low-income families, or from immigrant families (OECD, 2001). On the other side, empirical research on early indicators of school readiness and on early cognitive and social-emotional predictors of later academic achievement has increased our understanding about important prerequisites for a child`s successful transition to formal learning (e.g., Blair \& Diamond, 2008).

Based on findings stemming from international studies on students`outcomes and on structural differences in school systems (e.g., OECD, 2005, 2006), many OECD countries have made efforts aiming at improving pre-primary and primary education. It is now widely accepted that preschool education is an effective mean to deal with the large heterogeneity in young children, to promote children`s school readiness, to yield longer-lasting positive effects on children`s cognitive and social-emotional development, and to increase equal opportunities for a successful transition into formal school for children from diverse socio-economic backgrounds (e.g., Sammons, Elliot, Sylva, Melhuish, Siraj-Blatchford, \& Taggart, 2004; Sylva, Taggart, Siraj-Blatchford, Totsika, Ereky-Stevens, Gildena, \& Bell, 2007). Systematic and scientific evaluations of school models that specifically allow a flexible and individualized transition into school (based on a child's competencies and not based on chronological age), however, are still rare.

The goal of the present paper is to present empirical evidence on one newly installed school transition model in Switzerland, in which 4- to 8-year-olds are integrated in one class (mixed-grades classes; "Basisstufe") by systematically comparing children in traditional 
kindergarten and primary school classes with age mates enrolled in such mixed-grades classes. While in a previous research report on this longitudinal study, the focus had been laid on developmental progression in the core dimensions of executive functions as well as on precursors of academic skills (first and second measurement point; see Neuenschwander, Röthlisberger, Michel, \& Roebers, 2011; Roebers, Röthlisberger, Cimeli, Michel, \& Neuenschwander, 2011), emphasize in this paper will be put on children's school progression, aspects of self-regulated learning, and academic outcomes (third measurement point). In the following paragraphs, we will first provide more details on the aims and the existing evaluations of these unique mixed-grades classes, and will then move on outlining the theoretical rationale for the outset of our study.

\section{Individualized and Flexible Transition to Formal Learning}

One of the major aims of the pilot school project " 4 to 8 " ("Basisstufe") was to provide an educational, institutional context for young children in which a smooth and gradual transition into formal learning could take place without leaving any child behind (no segregation, no grade-retention). Children aged 4 to 8 years of age were enrolled together in one class with two teachers being present in $50 \%$ of the time allowing both individualized instructions and teaching in small, relatively homogenous groups of students. At the same time, play and social learning was strongly emphasized in these mixed-grades classes, purposefully giving children of any age ample opportunities to engage in activities of the own choice. There is one thorough and detailed formal evaluation of the implementation of these mixed-grades classes by Vogt, Zumwald, Urech, and Abt (2010). In this study, more than 80 classes (from the school project and traditional kindergarten and elementary school classes) were followed over an extended time period embracing 4 assessments that included questionnaires, video-recorded systematic class observations, and structured interviews of teachers, parents and children. 
As to issues of class management, instructional methods and learning settings, results of that evaluation revealed that the two teachers present in these project classes provide substantially more and different forms of learning, playing, and instruction throughout the school day compared to the traditional classes. Depending on the school subject, individualized learning activities or small-group instructions seem to be dominant. Topics in science, for example, were found to be especially suited for individualized work, while mathematics and literacy were more often taught in small, relatively homogenous groups of students (followed by individualized work). As to children's play and social development, 6and 7-year-olds in the mixed-grades classes were found to spent significantly more time in child-centered free play in comparison to children enrolled in traditional $1^{\text {st }}$ or $2^{\text {nd }}$ grade classes. At the same time, 4- and 5-year-olds in the mixed-grades classes spent less time playing than children in the traditional kindergarten classes but were more often engaged in individualized tasks covering a circumscribed topic (mainly in the domains of science or music), or instructed in small groups without individualization (precursors of mathematics and literacy; Vogt et al., 2010).

As to children's outcomes, Moser and Bayer (2010) undertook the students' outcome evaluation and documented that mixed-grades classes were indeed successful in terms of fully integrating children with special needs. In terms of academic outcomes, the authors found that mean performance in mixed-grades classes was equally good as in traditional school settings (see also Liebers, 2008, for similar results on mixed-grades classes in Germany). These findings were not necessarily expected and were positively evaluated since (a) the mixedgrades classes integrate children with special needs, developmental delays and disorders, resulting in an increased probability for poorer mean performance, and (b) 6- and 7-year-old children in the mixed-grades classes were documented to spent more time playing (Vogt et al., 2010). Relatedly, parents and teachers alike gave higher ratings of social skills for children in mixed-grades classes (Vogt et al., 2010). With respect to precursors of academic 
performance, Roebers and colleagues (Roebers et al., 2011) showed that both Swiss traditional kindergarten and theses newly installed mixed-grades classes equally well fostered development in early mathematics and literacy (counting, simple addition, verbal fluency).

With respect to children's self-perceptions of competence, an important student's characteristic (Helmke, 1998; Marsh \& Martin, 2011), the three existing studies concerning the effects of mixed-grades classes on children's (pre-)academic self-concept have produced contradictory results. In one study, the younger students in mixed-grades classes have evaluated their performance less positive than children in same-grade classes (Neuenschwander et al., 2011). Moser and Bayer (2010) as well as Liebers (2008) found no group differences in children's self-concept between mixed-grades and same- grade class members. Results seem to strongly depend on children's age within the mixed-grades classes (allowing more upward or more downward comparisons) and have been interpreted as confirming effects of group-reference, social comparisons, and positive feedback, already present in this young age (e.g., Gabriel, Kastens, Poloczek, Schoreit, \& Lipowsky, 2011; Leflot, Onghena, \& Colpin, 2010; Marsh \& Parker, 1984).

\section{Theoretical Background}

Given that the mixed-grades classes proved to provide more individualized and small group learning opportunities, the theoretically important question arises whether and to what extent such a learning context may foster development of self-regulatory skills. Other curricula emphasizing individualized learning have been found to yield positive effects on self-regulatory skills (e.g., Lillard, 2012; Lillard \& Else-Quest, 2006; Perry, 1998). In this report, self-regulatory processes embrace executive functions (i.e., inhibitory control, cognitive flexibility, and working memory), procedural metacognition (i.e., metacognitive monitoring and control), but also a student's self-perceived competence (i.e., academic selfconcept), as these constructs have been found to be theoretically and empirically related to 
each other and to early academic outcomes (Efklides, 2011; Roebers, Cimeli, Röthlisberger, \& Neuenschwander, in press). As self-regulatory processes are now widely considered as an important individual differences variable (a correlate and/or predictor) for explaining achievement differences throughout the life-span (e.g., Baumert et al., 2001; Blair \& Diamond, 2008; Moffitt et al., 2011), an innovative and integrative framework of selfregulated learning (and the ontogeny thereof) was chosen as theoretical background of the present study.

Integrating existing theories of self-regulated learning, Efklides (2011) outlines a model in which affective and cognitive individual characteristics on the person level, such as self-concept, abilities, and motivation, interact with the demands of any given task. Individual differences in these aspects explain how a student decides to deal with any given task (i.e., how much study time to allocate, how much effort to invest, which strategies to adopt; i.e., top-down processes). At the same time, students make subjective (metacognitive) experiences during task mastery (e.g., experiencing uncertainty, making performance predictions; i.e., monitoring) which in turn trigger control processes (e.g., adaptation of strategies as bottom-up processes). For instance, a student with a high (as opposed to a low) self-concept (SC) will experience a task as challenging (as opposed to threatening), will invest more time in finding the correct solution (thus make more experiences), will be certain about the correctness of his or her solution (as opposed to uncertain), and will internally attribute success to effort and/or ability (as opposed to luck or an easy task) leading to an even higher SC. Thus, the model integrates conceptualizations of self-regulated learning that focus strongly on information processing (Boekaerts, 1999; Nelson \& Narens, 1990; Pressley, Borkowsky, \& Schneider, 1989 ) and those including a broader, motivational perspective (Winne \& Hadwin, 2008; Zimmerman \& Moylan, 2009). Of importance for the present study, metacognitive (Efklides, 2011) and executive processes (i.e., cognitive flexibility, inhibition, working memory; Borkowsky, Chan, \& Muthukrishna, 2000) taking place while mastering a task are considered 
as the missing link between student's on-task behavior and long-term outcomes of selfregulated learning. In other words, it is only through monitoring and executive control processes that strategies can be initiated, adapted, and/or terminated.

Clearly, such a broad and overarching theoretical framework is difficult to confirm as a whole. However, Efklides and colleagues provide empirical evidence how self-concept, affect, and mood systematically influence task-specific self-regulatory processes and learning outcomes (e.g., Efklides, 2006; Efklides \& Petkaki, 2005). Roebers and colleagues (Roebers et al., in press) followed-up on these results and were able to show for $2^{\text {nd }}$ graders that individual differences in children's self-concept were linked to metacognitive monitoring (confidence judgments in a spelling test), both cross-sectionally and longitudinally (i.e., topdown self-regulatory processes). Adequate metacognitive control skills, in turn, were substantially associated with executive functions, also both cross-sectionally and longitudinally (i.e., bottom-up self-regulatory processes). Together, these findings demonstrate the complex, bi-directional interplay between characteristics of the person and processes triggered through the subjective experiences an individual makes while mastering the task at hand.

\section{The Present Study}

In the present study, a multi-perspective approach on the above mentioned mixedgrades classes' school project was realized, with a special focus on the above mentioned diverse aspects of self-regulatory processes. A systematic comparison of these two distinct school context is already informative on the descriptive level, as mixed-grades classes have only rarely been formally installed and evaluated but are being discussed by social policy makers since the 1960s ("ungraded elementary schools"; Gutiérrez \& Slavin, 1992). Of equal importance, however, is the theoretically motivated question whether enrollment in mixed- 
grades classes implies a psychologically distinct learning context that may lead to differences in self-regulatory processes.

Thereby, the present study is highly explorative in nature and thus, no specific hypotheses were generated. In order to provide first insights into the effects of a school system aiming to allow a gradual transition into formal learning, an issue that is currently of international interest (see Daseking, Oldenhage, \& Petermann, 2008, for an overview), the focus is laid on students' characteristics discussed in the context of self-regulatory processes. As to academic outcomes and children's early school progression, we aimed at following up on Moser and Bayer's report (2010). Even more importantly, however, we aimed to extend the literature by exploring children's self-regulatory skills as - theoretically - the mixedgrades classes may yield small but detectable effects due to the formal organization of these classes in which instruction in small groups of children and individualized work phases were more frequently realized than in the traditional classes (Vogt et al., 2010). These aspects were children's self-concept (Harter, 2012), executive functions (Blair \& Diamond, 2008;

Bukowsky et al., 2000; Diamond, Barnett, Thomas, \& Munro, 2007), as well as metacognitive monitoring and control (Efklides, 2011; Roebers at al., in press; Schneider \& Lockl, 2008). Because different school contexts may not only affect the level of skills but also the interplay of these, theoretically related student's characteristics, the associations between the included variables as a function of learning context were also addressed.

\section{Method}

\section{Sample}

The study presented in this paper included $N=230$ children, $N=106$ boys and $N=$ 124 girls. At the third measurement point of which data is reported here, the mean age of the younger age group (called "younger group", $n=140)$ was 7 years and 7 months $(M=68.9$ 
months; $S D=4.4$ months) and the mean age of the older age group (called "older group", $n=$ 90) was 8 years and 6 months $(M=78.9$ months; $S D=4.0$ months $)$. There were two different primary school contexts: within each age group, half of the children (younger group: $n=70$, older group: $n=45$ ) attended traditional Kindergarten (first and second measurement point) or primary school classes (third measurement point reported here), whereas half of the children attended "mixed-grades classes" (description of the different school contexts see above).

\section{Insert Table 1 about here}

A careful individual $(1: 1)$ matching was initially done between kindergarten children and mixed-grades class children at $T_{1}$ in order to specifically address schooling effects (Neuenschwander et al., 2011). Matching variables were sex, age in months, language skills, non-verbal intelligence, as well as parents`socio-economic background (SES). As Table 1 shows the two age groups of children within the two school contexts did not differ concerning the matching variables. In the initial report of this study $\left(\mathrm{T}_{1}\right), n=164$ five-year-olds $(n=84$ in each school context) and $n=114$ six-year-olds ( $n=57$ in each school context) were included (see Neuenschwander et al., 2011). One year later at the second measurement $\left(\mathrm{T}_{2}\right)$, there were $n=160$ now 6 -year-olds and $n=104$ now 7-year-olds $(n=80$ and $n=52$, in each of the two school contexts, respectively; Roebers et al., 2011). In the present report, only data of the third and last measurement $\left(\mathrm{T}_{3}\right)$ is presented with $n=140(n=70$ in each school context $)$ now 7-year-olds and $n=90$ now 8-year-olds ( $n=45$ in each school context; see above). Whenever a participating child moved out of the study's reach, his or her experimental twin was also removed from the analyses, leading to an exclusion of $n=52$ children. In other words, $82 \%$ of the matched sample completed all three measurement points. Importantly, there were no systematic differences between children who dropped out (or were excluded from the analyses) and those who completed the study. 
The traditional pre-primary and primary school system in Switzerland comprises two years of child-centered, play-oriented kindergarten for 5- and 6- year-old children. School enrollment into the formal- instructional primary school is solely based upon the child`s date of birth according to a cutoff date. As described in more detail in the introduction, the innovative school transition school project called "mixed-grades classes" (in German "Basisstufe"), in contrast, integrates the two years of kindergarten and the first two primary school years. This school context dissolves the didactical segregation of the traditional school system providing a "playful learning” and offers a flexible, ideally individual school transition according to the child's development. The mixed-grades classes consist of children from four different age groups (4- to 8-yr-olds) and are run by two professionals (one kindergarten and one primary school teacher) working in team-teaching and imply about $50 \%$ of individualized, formal instruction in the major school domains, emphasizing basic academic skills (Vogt et al., 2010). The teachers had received specialized training before starting their work in the mixed-grades classes and were closely supervised by the school authorities who ran the school project. Parents were not able to choose which school context their child would attend; rather, the mixed-grades classes were installed by the school authorities and children were enrolled based on the school district and their home address.

Children came from diverse socio-economic backgrounds, ranging from lower working-class to upper middle-class families; $n=39$ children (19\%) were immigrants and non-native (German) speakers, with either Caucasian or Asian ethnicity; all participants were sufficiently fluent in German language. The participating children at the end of the study $\left(\mathrm{T}_{3}\right)$ were distributed over 81 different kindergarten, primary school or mixed-grades classes. Informed parental consent was obtained prior to the study. Due to sickness or due to pragmatic reasons (time constraints of the schools) few tasks were not completed by all participants; the slightly varying numbers of data points in the analyses are indicated by the degrees of freedom. 


\section{Procedure}

Data included in the present paper were collected in the last quarter of the school year. Children were seen by a trained experimenter individually in a separate room in their institution. Each child participated in two sessions of approximately 30 minutes each, with the two sessions being 4 to 8 days apart. The order of the tasks was counterbalanced.

Additionally, a small group testing of 3 to 6 children for the standardized school achievement tests including the metacognitive monitoring and controlling measurement was realized. In the end of each assessment, children and teachers received a small present for participation.

\section{Measures}

School Progress and Achievement. Data on children's school progress (special need class, grade etc.) was derived directly during the testing through the experimenter and validated through the child's teacher. School achievement in the domain of math, reading and spelling was measured with standardized school achievement tests. For Literacy Achievement three tasks were used, two of them measuring reading and one of them measuring spelling. In the Reading Speed test children had to choose the corresponding picture to a written word (max. 70) out of four pictures representing similar sounding items within a time limit of 5 minutes (WLLP; Küspert \& Schneider, 1998 test-retest reliabilities: .75 - .81). In the Reading Comprehension test children were asked to judge the accuracy of a sentence (max. 140) containing between 3 and 9 words, within a time limit of 3 minutes (SLS; Mayringer \& Wimmer, 2005, parallel- test reliability: .90). The dependent variable of the two reading tests was the number of correct answers, i.e., the sum of correct identified word-picture pairs and the sum of correct identified sentences. In the Spelling test children had to write down a sentence that was read aloud by the experimenter without time limit (adaption of HSP; May, 2002). The dependent variable of the spelling test was the number of correct graphemes out of max. 27 correct graphemes. 
For Mathematical Achievement three tasks were administered (adapted and originally from HRT 1-4; Haffner, Baro, Parzer, \& Resch, 2005). The first math task, Equations, consisted of max. 40 items in which quantities had to be compared within 2 minutes. Children had to fill in an equal $(=)$ or a correct unequal sign $(<$ or $>$ ) between two quantities. For getting the right solutions sometimes number knowledge (e.g., $83>38$ ), sometimes estimating and calculating (e.g., $17+17>17$ ) was required. As dependent variable an “equation score" was calculated (number of correct equation solutions minus number of incorrect equation solutions divided through 2). In the second math task, Sequence of Numbers, max. 20 progressions of numbers had to be completed by the children within 3 minutes. For the third math task, Additions and Subtractions, children were given max. 12 basic arithmetic tasks including addition and subtraction signs and varying in their length (min. 2 numbers- max. 5 numbers) to be completed in 2 minutes and 30 seconds. For the last two mathematical achievement tasks the dependent variables were the number of correct solutions within the given time limit.

Self-Concept. A rating scale was used for measuring Academic Self-Concept (similar versions: Helmke, 1998; Nicholls, 1978; Nurmi \& Aunola, 2005; Roebers \& Schneider, 1999; Röthlisberger \& Michel, 2009; Stipek \& Daniels, 1988). Children were given a sheet of paper depicting vertical rows of 25 schematic faces and explained that theses rows of faces represented their classmates. The experimenter further explicated that on top of each row, there is the student that is best in the specified area of interest (e.g., counting), on the bottom of that same row, there is the classmate that is worst in this domain. The "children" on the middle line represented a child that is "average" in this domain. Participants were then instructed to mark the one face in each row that best represented their own rank, for each of the domains separately. For the analyses reported below, a numerical value was assigned to each of the positions ( 1 - 25), with higher values indicating a more positive view of the child`s 
own performance in the single domains. The dependent variable representing the child`s academic self-concept at the third measurement was calculated as the average value of his self-ratings in "counting", "letter knowledge", "reading and spelling", and "basic arithmetic skills" (Cimeli et al. in press; Roebers et al., in press).

Executive Functions. Executive functions were operationalized following the conceptualization of executive functions in the three processes "inhibition", "updating" and “shifting" of Miyake et al. (2000).

An adapted version of the Fruit Stroop task of Archibald and Kerns (1999) was used to assess "inhibition". Four pages displaying each 25 items were presented to the child: the first page displayed 25 colored squares and children were required to name the colors as quickly as possible. On the second page, four different fruits and vegetables in their natural original color were presented (congruent trial), followed by a third page displaying the same fruits and vegetables in black and white and children were required to name the original colors. The fourth and final page showed the fruits and vegetables colored incorrectly and children were required again to name the original color (incongruent trial). The dependent variable was the measure of interference according to Archibald and Kerns '(1999) formula with smaller values (sec) indicating better performance in terms of resistance to interference (Roebers et al., 2011).

As a measure of "updating", a Backwards Color Recall task similar to a backwards digit span task was used (Schmid, Zoelch, \& Roebers, 2008; Schumann-Hengsteler et al., 2010). In this task, the relevant information (colors) has not only to be remembered but also manipulated (recall in reversed order). Embedded in a cover story of a dwarf, children were presented a sequence of colored discs on a computer screen and asked to recall the sequence in the reversed order. Only colors with monosyllabic (German) names were selected. Duration of disc presentation was $1 \mathrm{sec}$. Testing began with a two- item trial and there were three trials 
per level. The transition to the next level was only possible if two of three trials were correctly recalled. The dependent variable was the number of color sequences correctly recalled in the reversed order (Roebers et al., 2011).

"Shifting" was assessed with the computerized Cognitive Flexibility task developed in our lab (Michel, Kauer, \& Roebers, 2011; Roebers \& Kauer, 2009; Roebers et al., 2011). There were two categories of stimuli (two different kinds of fish) presented simultaneously on the right and left side of the computer screen. Children were told that their task was to feed two families of fish alternately, i.e., to feed a member of one family in the first trial and a member of the other family in the next trial. Because each time two fish (one of each category) appeared on the screen with randomly changing sides, it was the child's task to decide whose turn it was to be fed by remembering which category of fish had been fed in the previous trial. Fish were fed by pressing a button on the side corresponding to their appearance of the computer screen (right or left). There were 46 trials, with a short break including positive feedback after half the trials. Interstimuli intervals varied from 300-700 msec. The dependent variable was the proportion of correct responses (accuracy). In order to get comparable metrics across the different components of executive functions, all three dependent variables of the processes inhibition, updating and shifting were z-standardized. A composite score as the mean of these three z-standardized measures then served as dependent measure for executive functions.

Metacognitive Monitoring and Control. Metacognitive monitoring and control were measured using a multi-phase task adapted from previous experimental studies (Krebs \& Roebers, 2009; 2010; Roebers \& Fernandez, 2002; Roebers, Schmid, \& Roderer, 2009): in the first phase of this task, children were presented with 18 schematic pictures of simple objects and animals (e.g., "Hund"- dog; "Fliege" - fly; "Handtuch" - towel; "Fahrrad" - bicycle) and instructed to write the corresponding word on the line beside the picture. Items were selected based on intensive piloting ensuring varying degrees of item difficulty (mean proportion 
correct across children $=.65$; range: $.24-.90$, each 6 easy, average, and difficult items, respectively). After having completed the spelling test with a black pencil, children were given a blue pencil and were asked to rate their confidence that the word written down was spelled correctly. Before they were allowed to start with the confidence ratings, children were familiarized with the 7-point-Likert confidence scale (Roebers et al., 2009). Explanations and labels were given for each point on the scale and practice questions were asked. Children understood the use and rationale of the scale with ease. The experimenter made sure that children gave confidence judgments to every word they had written down, resulting in $N=18$ confidence ratings. For the third and last phase of this task, the blue pencils were exchanged into red ones and children were allowed to cross-out previously written words if they believed they were spelled incorrectly. Children were not obliged to cross-out any word, only if they wished. The dependent variables for monitoring were the averaged confidence judgments considering the easy, average, and difficult items separately and distinguishing between average confidence judgment of correctly spelled words and average confidence judgment of incorrectly spelled words within these groups of items. The dependent variable for metacognitive control was an aggregate score for adequate control behavior in the last phase of the experiment, that is, summing the number of adequately withdrawn (words that had been spelled incorrectly and then crossed-out) and adequately maintained words (words that had been spelled correctly and had then been maintained in the controlling phase). The reliability and validity of this approach is documented and described in even more detail in Roebers et al. (in press).

\section{Results}

Insert Figure 1 about here

Young Students`School Progression as a Function of School Context. Figure 1 illustrates children`s school progress at the last measurement of the present study. In the traditional 
school system, $2 \%$ of the children faced grade retention either by repeating a grade or by having their school enrollment postponed for a year, and an additional 11\% were segregated into special needs classes, while in the mixed-grades classes, there was no such a case of grade retention or segregation into special need classes during our study period. Noteworthy, at the same time, the frequency of children progressing more quickly than scheduled through the first elementary school years was much higher in the mixed-grades classes than in the traditional school system (19\% vs. 3\%).

\section{Insert Figure 2 about here}

Academic Performance. In the following section, we present group differences as a function of age and school context. Because the variables used for the matching nevertheless contributed to individual differences in the outcome measures, we will additionally report results of analyses of covariance in which individual differences in the matching variables were controlled for. Figure 2 presents mean performance in the domain of literacy (reading speed, reading comprehension, and spelling) as a function of age group and school context. Separate analyses of variance were conducted with age group and school context as betweensubject factors. Results for reading speed of words revealed no significant main effect of school context, but a significant main effect of age group, $F(1,226)=90.21, p<.001, \eta^{2}=$ .29. The age group $\times$ school context interaction was not significant. Controlling for the background variables depicted in Table 1 did not change this overall pattern of results; however and as expected, the main effect of age group was no longer significant $(F<2)$.

The corresponding analysis of variance on reading comprehension of sentences also revealed no significant main effect of school context, but a significant main effect of age group, $F(1,226)=87.55, p<.001, \eta^{2}=.28$. For reading comprehension, however, the interaction between age group and school context interaction was significant, $F(1,226)=3.94$, $p<.05, \eta^{2}=.02$. After controlling for the background variables, the age effect was no longer 
significant $(\mathrm{F}<4)$, but the interaction between school context and age remained significant $\left(F(1,219)=6.60, p<.05 ; \eta^{2}=.04\right)$. As can be seen in the middle panel of Figure 2 , the interaction was due to the fact that in the younger age group children did not differ between the two school contexts $(F(1,138)=0.30, p>.05)$, while within the older age group, children in mixed-grades classes showed significantly superior performance compared to children in the traditional school system.

The 2 (age groups) x 2 (school context) analysis of variance with spelling performance as dependent measure revealed no significant main effect of school context but a significant main effect of age group, $F(1,225)=88.46, p<.001, \eta^{2}=.28$. Again, the interaction between age group and school context was significant, $F(1,225)=4.15, p<.05, \eta^{2}=.02$. Controlling for the background variables did decrease the size of the main effects (main effect of age: $F(1$, $218)=8.38, p<.01$; interaction age $\mathrm{X}$ school context: $F(1,218)=6.51, p<05)$, the overall pattern, however, remained the same. Follow-up tests on the interaction revealed that the interaction between age and school context was due to the fact that, among the younger age group, children in the traditional school system did not differ from children in the mixedgrades classes, whereas in the older age group, children in the mixed-grades classes significantly outperformed children in the traditional school system. Taken together, the inclusion of two age groups consistently revealed substantial improvements in aspects of early literacy clearly demonstrating the reliability of the underlying data. Of central importance for the main research question addressed here are the findings of superior performance of children in the mixed-grades classes with respect to reading comprehension and spelling, advantages that only emerged in the older age group.

Insert Figure 3 about here

With respect to mathematical skills, Figure 3 presents the mean performance in the three measures of early arithmetic skills as a function of age and school context. Separate 
analyses of variance with the three dependent variables were conducted. Results for equation performance as dependent measure revealed no significant main effect of school context but a significant main effect of age group, $F(1,226)=62.87, p<.001, \eta^{2}=.22$; the interaction between age group and school context was not significant. Controlling for the background variables (depicted in Table 1) decreased the age effect somewhat $(F(1,187)=5.28, p<.05)$ but did not change the overall pattern of results. A corresponding analysis of variance with sequences performance as dependent measure revealed no significant main effect of school context but a significant main effect of age group, $F(1,226)=71.21, p<.01, \eta^{2}=.24$. Again, the interaction between age group and school context was not significant. The analyses of covariance controlling for the background variables revealed that also the age effect was no longer significant $(F(1,219)=2.48, n s)$. Finally, the analysis of variance with arithmetic performance (additions/subtractions) as dependent measure also revealed no significant main effect of school context but again a significant main effect of age group, $F(1,226)=64.68, p<$ $.001, \eta^{2}=.22$, that remained significant after controlling for the background variables $(F(1,187)=11.41, p<.01$. As was the case for equations and sequences, there was no significant interaction between age group and school context for addition/subtraction.

Insert Figure 4 about here

Academic Self-Concept. An analysis of variance was conducted with academic self-concept as dependent variable, age group and school context as between-subjects factors. Inspection of the left panel of Figure 4 suggests that all children`s self-concept was positively biased, independent of school context. Results revealed only a small main effect of age group $\left(F(1,225)=3.93, p<.05, \eta^{2}=.02\right.$, that remained relatively unchanged when controlling for the included background variables $(F(1,219)=5.79, p<.05$. Neither the effect of school context nor the interaction between age and school context were significant.

Executive Functions. The right panel of Figure 4 presents performance in the standardized composite measure of executive functions as a function of age and school context at the third 
measurement of the present study. The conducted analysis of variance revealed a strong main effect of age group, $F(1,225)=39.23, p<.001, \eta^{2}=.15$ (that was negligible after controlling for the background characteristics, $F<1$ ), but no main effect of school context and no interactions.

Insert Figure 5 about here

Metacognitive Skills. Two aspects of metacognition was included in the present study: children`s ability to adequately rate their confidence that a given word was spelled correctly (monitoring) and to efficiently correct errors in their own spelling (control). Figure 5 depicts mean performance in metacognitive monitoring and controlling as a function of age and school context. When monitoring of easy and average words was considered, there were no differences between the two school contexts, $F_{\text {easy }}(1,150)=0.11, n . s ., F_{\text {average }}(1,197)=0.80$, n.s.. However, an analysis of variance on monitoring the difficult items (confidence judgments) with age group and school context as between-subjects factors and correctness of spelling as within-subject factor revealed a significant main effect of correctness of spelling, $F(1,95)=15.35, \mathrm{p}<.001, \eta^{2}=.14$, and of school context, $F(1,95)=7.72, p<.01, \eta^{2}=.08$, but no main effect of age and no interaction between age group and school context. This main effect of school context remained significant even after controlling for the background variables $(F(1,77)=8.13, p<.01)$. As can be seen in the left panel of Figure 5 , all children adequately gave significantly lower confidence judgments for words they had spelled incorrectly and children in the mixed-grades classes gave overall lower confidence ratings than children in the traditional classes. Although inspection of Figure 5 suggests that the older children in the mixed-grades classes showed a substantially more pronounced metacognitive differentiation between correctly and incorrectly spelled words (appear especially unsure for incorrectly spelled words), the interaction was not significant (after controlling for the background variables: $F<1$ ). 
As to adequate metacognitive controlling, the right panel of Figure 5 shows average performance with respect to children`s crossing out of incorrectly spelled words and maintenance of correctly spelled words (sum score mirroring adequate controlling). Results of the analysis of variance with age group and school context as between-subject factors revealed no main effect of school context but a main effect of age group, $F(1,226)=48.77, p$ $<.001, \eta^{2}=.18$, and also an age group by school context interaction, $\mathrm{F}(1,226)=3.46, p<.10$, $\eta^{2}=.02$. However, after controlling for the background variables, only the age effect remained significant $(F(1,187)=4.78$. As was found for monitoring, inspection of Figure 6 suggests an advantage of the older children in the mixed-grades classes; the differences were, however, not statistically reliable.

Insert Table 2 about here

Interrelations between the included variables. As a final aspect, associations between school achievement measures and the included student's characteristics will be presented as a function of school context. By these means, an integration of the included variables was attempted, based on the theoretical notion that a school context may also influence the interplay of single self-regulatory processes and their impact on academic outcomes. Table 2 presents partial correlations controlling for age. In order to simplify presentation of results, data was aggregated into larger constructs. Inspection of Table 2 reveals that the overall pattern of associations does not differ substantially between the two school contexts. Among the self-regulatory processes, metacognitive skills and executive functions (but not selfconcept and mathematics) are significantly related to both mathematic and literacy achievement in both school contexts. Self-concept, metacognition, and executive functions do not seem to be substantially interrelated, but mathematics and literacy are.

\section{Discussion}


The major aim of the present study was to explore children's early school progress into formal learning, their academic performance, and a variety of self-regulatory processes known to be related to learning outcomes as a function of school context. Specifically, individual differences in students' academic self-concept, executive functions, and metacognitive processes (monitoring and control) were included. A strict individual matching procedure controlling for important background characteristics (i.e., SES, non-verbal intelligence, speed of processing, language skills, age, and sex) was realized resulting in two sub-samples of children attending either traditional classes or mixed-grades classes.

Marked group differences between the two school contexts emerged when children`s school progression was addressed. In the mixed-grades classes, a higher proportion of children progressed faster towards higher grades compared to children attending the traditional school system with rigid transition scheduling (see also Moser \& Bayer). In our view, these results suggest that one central aim of installing the mixed-grades classes aiming to account for the pronounced developmental heterogeneity in young children `s readiness for school was achieved. It appears from our results that students benefitted from the opportunities to flexibly reach their achievement goals and were then allowed to progress. At the same time, the relatively high percentages of children that were mostly segregated into special needs classes from the traditional school system together with the absence of decelerated progressions in the mixed-grades classes show that the mixed-grades classes successfully facilitated children`s transition into formal school learning, indicating a noticeable school's readiness for children in the case of the mixed-grades classes. Thus, it appears that the mixed-grades classes can provide individualized learning opportunities for the weaker students and at the same time avoid early stigmatizations and their negative psychological consequences (Liebers, 2008). 
Although heterogeneity within the single classes was larger in the mixed-grades classes compared to the traditional school, we found by and large no group differences in academic performance, confirming previous studies (Liebers, 2008; Moser \& Bayer, 2010). This overall pattern of similar group performance generalized to the subcomponents of math (i.e., Equations, Sequences, and Addition/Subtraction) and literacy (reading speed). Interestingly, however, the older children in the mixed-grades classes had an advantage over their age-mates in the traditional classes in terms of reading comprehension and spelling. Admittedly, these differences were small and not necessarily of practical relevance. Our findings are in line with previous results and show that mixed-grades classes are at least not disadvantageous for children (Gutiérrez \& Slavin, 1992; Moser \& Bayer, 2010). Overall, the present results regarding academic performance argue for a successful implementation of the mixed-grades classes and suggest that the impact of an individualized learning context on academic achievement is rather small, domain-specific, and age-dependent. The description of such outcomes despite many open questions regarding the precise learning opportunities for a single child may serve as a starting point for future research.

Children`s self-concept proofed to be unaffected by the school context, although the two school systems differ in the composition of their classes, a factor that is generally known to affect children`s self-evaluations by means of social comparisons ("big-fish-little-pondeffect", see Marsh \& Parker, 1984). Possibly, children in the mixed-grades classes engage preliminary in social comparisons within a homogenous learning subgroup like the traditional school children do and/or in intra- individual comparisons which - in this age window - is dominated by children's factual competence gains (Cimeli, Neuenschwander, Röthlisberger, \& Roebers, in press; Nicholls, 1984). Notably, a similar self-concept in traditional and mixedgrades class children is not in line with what has been reported about possible emotional and motivational disadvantages of early academic environments. In earlier studies, early academic learning had been found to negatively affect children's self-concept and their learning 
motivation (Elkind, 1981, Stipek, Feiler, Byler, Ryan, Milburn, \& Salmon, 1998). Against this background, our results of comparable academic performance and - at the same time - a very positive self-concept on the side of the mixed-grades class children are promising. They might also suggest that more research is needed to challenge the conventional wisdom that a combination of early academic environments and positive emotional and motivational development is not possible (Stipek et al., 1995).

As was expected, there were no effects of the school context in our aggregated measure of executive functions. Although at different time points small and age-dependent advantages in executive functions in the mixed-grades classes had been reported (Neuenschwander et al., 2011), the general view of executive functions following mainly biologically predetermined developmental trajectories seems to be confirmed by the present findings (Skibbe, Connors, Morrison, \& Jewkes, 2011). Environmental factors known to improve executive functions may be very similar for children in the two school contexts. Such factors might be classroom rules, routines, and teachers' instructions emphasizing children's autonomy (e.g., Skibbe et al., 2011). It is also possible that we did not include the most sensible age range in which differences in learning context may yield substantial effects (Diamond et al., 2007).

With respect to metacognitive monitoring and controlling, the present study offers preliminary evidence that an individualized learning context may yield to small (and possibly age-dependent) advantages in these higher-order cognitive processes. As these self-regulated processes are considered as central for both, learning progress in the short and in the long run, even small and yet statistically non-reliable differences favoring children in the mixed-grades classes may stimulate further research into this direction. Although we cannot draw firm conclusions and this results needs to be replicated, we are tempted to believe that the present 
data is the first to offer direct evidence for the claim that schooling, over extended periods of time, will influence metacognitive development (Lockl \& Schneider, 2008).

In line with the interpretation that differences in the learning environment may need more time to yield their effects is the finding of no substantial differences in the associations among the different included student's characteristics (see Table 2). We aimed to explore whether the learning context would affect the relative importance of single aspects of self-regulatory processes and/or on academic outcomes. This did not seem to be the case, as the differences in the partial correlations between the two subsamples were only subtle. The significant correlation among mathematical and literacy performance is in line with previous studies revealing substantial associations between academic domains in young elementary school children suggesting that domain-specific competence profiles yet have to develop (Helmke \& Weinert, 1999). The substantial overlaps between the included aspects of self-regulatory processes on the one hand, and the two school achievement measures, on the other hand, demonstrate the importance for self-regulatory processes for academic outcomes. Interestingly, young children`s self-concept (embracing self-concept in literacy and mathematics) appeared to be already relatively domain-specific as weaker associations with academic performance were found compared to the other included variables (Marsh \& Martin, 2011). Finally, the present data confirmed recent theoretical accounts and empirical findings with respect to executive functions being empirically distinct from metacognition (see Best \& Miller, 2011; Roebers et al., in press).

Overall, there do not seem to be strong and longer-lasting positive or negative effects of mixed-grades classes in comparison to the traditional school system. Yet, the theoretical assumption that the learning environment may yield - sooner or later - effects on the students` outcomes including self-regulatory skills individual cannot be rejected. This is because (a) the differences between the learning contexts may have been too subtle, or (b) children did not 
yet spent enough time in their learning context for effects to emerge. Both interpretations are supported by the bulk of studies documenting positive effects of training, with focused, individualized or adaptive interventions (i.e., massed practice) relatively consistently and positively affecting the targeted domain (e.g., Röthlisberger, Cimeli, Neuenschwander, \& Roebers, 2012; Thorell, Lindquist, Bergman, Nutley, Bohlin, \& Klingberg, 2009). However, trainings are costly and difficult to implement in regular school days, transfer effects to nontrained processes sometimes difficult to obtain, and benefits may vanish over time (Hager \& Hasselhorn, 2000; Hasselhorn \& Mähler, 2000). Pursuing this line of argumentation, it may thus for pragmatic reasons still be worthwhile to optimize classroom processes and learning environments. Further research would certainly be worthwhile.

There are a number of limitations of the present approach that should be discussed at this point: Although there was a thorough evaluation of the implementation of the mixed-grades classes by the individual teachers, we do not have any data on instructional processes, individual differences in classroom organization and management, making it difficult to draw firm conclusions about underlying processes allowing the mixed-grades class students to undergo a typical academic and self-regulatory development as indicated by our results. But, the present study nevertheless constitutes one of very few attempts to gain detailed insights into children`s outcomes with the aim to fuel future research in this domain. Undoubtedly, early education and a smooth, child-oriented transition into formal learning are contemporary issues that deserve more research attention. Combining classroom and instructional processes with children`s outcomes in a multi-level analyses is certainly a necessary next step when trying to understand effects of a learning environment on children`s academic outcomes. 


\section{References}

Archibald, S. J., \& Kerns, K. A. (1999). Identification and description of new tests of executive functioning in children. Journal Child Neuropsychology, 5, 115 - 129. doi: 10.1076/chin.5.2.115.3167

Baumert, J., Klieme, E., Neubrand, M., Prenzel, M., Schiefele, U., Schneider, W., .. . Weiß, M. (Eds.). (2001). Basiskompetenzen von Schülerinnen und Schülern im internationalen Vergleich. [Basic competencies of students in an international comparison]. Opladen, Germany: Leske + Budrich.

Best, J. R., \& Miller, P. H. (2010). A developmental perspective on executive function. Child Development, 81, 1641-1660. doi: 10.1111/j.1467-8624.2010.01499.x

Blair, C., \& Diamond, A. (2008). Biological processes in prevention and intervention: The promotion of self-regulation as a means of preventing school failure. Development and Psychopathology, 20, 899-911. doi: 10.1017/S0954579408000436

Boekaerts, M. (1999). Self-regulated learning: Where we are today. International Journal of Educational Research, 31, 445-457. doi: 10.1016/S0883-0355(99)00014-2

Borkowski, J. G., Chan, L. K. S., \& Muthukrishna, N. (2000). A process-oriented model of metacognition: Links between motivation and executive functioning. In J. G. Borkowski \& J. D. Day (Eds.), Cognition in special children: Comparative approaches to retardation, learning disabilities, and giftedness (pp. 123-152). Norwood, NJ: Ablex.

Cimeli, P., Neuenschwander, R., Röthlisberger, M., \& Roebers, C. M. (in press). Das Selbstkonzept von Kindern in der Schuleintrittsphase: Ausprägung, Struktur und Zusammenhänge mit frühen Leistungsindikatoren [Self-concept of children at school entry: Mean level, structure and relations to indicators of early achievement]. Zeitschrift für Entwicklungspsychologie und Pädagogische Psychologie. 
Daseking, M., Oldenhage, M., \& Petermann, F. (2008). Der Übergang vom Kindergarten in die Grundschule - Eine Bestandsaufnahme. [The transition from kindergarten to elementary school - an appraisal]. Psychologie in Erziehung und Unterricht, 55, 8499.

Diamond, A., Barnett, W. S., Thomas, J., \& Munro, S. (2007). Preschool program improves cognitive control. Science, 318, 1387-1388. doi: 10.1126/science.1151148

Efklides, A. (2006). Metacognitive experiences: The missing link in the self-regulated learning process. Educational Psychology Review, 18, 287-291.

Efklides, A. (2011). Interactions of metacognition with motivation and affect in self-regulated learning: The MASRL model. Educational Psychologist, 46, 6-25. doi: $10.1080 / 00461520.2011 .538645$

Efklides, A., \& Petkaki, C. (2005). Effects of mood on students' metacognitive experiences. Learning and Instruction, 15, 415-431.

Elkind, D. (1981). The hurried child - growing up too fast too soon. Reading, Massachusetts: Addison-Wesley Publishing Company.

Gabriel, K., Kastens, C., Poloczek, S., Schoreit, E., \& Lipowsky, F. (2010). Entwicklung des mathematischen Selbstkonzepts im Anfangsunterricht - Der Einfluss des Klassenkontextes. [The development of elementary students `mathematical selfconcept - the influence of the class context]. Zeitschrift für Grundschulforschung, 3, $65-82$.

Gutièrrez, R., \& Slavin, R. E. (1992). Achievement effects of the nongraded elementary school: A best evidence synthesis. Review of Educational Research, 62, 333-376.

Haffner, J., Baro, K., Parzer, P., \& Resch, F. (Eds.). (2005). Heidelberger Rechentest- HRT 14: Erfassung mathematischer Basiskompetenzen im Grundschulalter. [Heidelberg test of arithmetic skills]. Göttingen, Germany: Hogrefe. 
Hager, W., \& Hasselhorn, M. (2000). Psychologische Interventionsmassnahmen: Was sollten sie bewirken können?. [Psychological interventions: What should effects should they have?] In W. Hager, J.-L. Patry, \& H. Brezing (Hrsg.), Handbuch Evaluation psychologischer Interventionsmaßnahmen: Standards und Kriterien (S. 41 - 85). Huber Verlag: Bern.

Harter, S. (2012). The construction of the self: Developmental and sociocultural foundations. New York, NY: Guilford Press.

Hasselhorn, M., \& Mähler, C. (2000). Transfer: Theorien, Technologien und empirische Erfassung. [Transfer: Theory, techniques, and empirical measurement] In W. Hager, J.-L. Patry \& H. Brezing (Hrsg.), Handbuch Evaluation psychologischer Interventionsmaßnahmen: Standards und Kriterien (S. 86 - 101). Huber Verlag: Bern. Helmke, A. (1998). Vom Optimisten zum Realisten? Zur Entwicklung des Fähigkeitskonzeptes vom Kindergarten bis zur 6. Klassenstufe [From optimism to realism? The development of the self-concept of ability from kindergarten to 6th grade]. In F. E. Weinert (Ed.), Entwicklung im Kindesalter (pp. 115-132). Weinheim, Germany: Beltz Psychologie Verlags Union.

Helmke, A., \& Weinert, F. E. (1999). Schooling and the development of achievement differences. In F. E. Weinert, \& W. Schneider (Eds.), Individual development from 3 to 12: Findings from the Munich longitudinal study (pp 176 - 192). Cambridge, UK: Cambridge University press.

Krebs, S. S., \& Roebers, C. M. (2010). Children's strategic regulation, metacognitive monitoring, and control processes during test taking. British Journal of Educational Psychology, 80, 325-340. doi: 10.1348/000709910X485719

Küspert, P., \& Schneider, W. (1998). Würzburger Leise Leseprobe (WLLP). Göttingen, Germany: Hogrefe. 
Leflot, G., Onghena, P., \& Colpin, H. (2010). Teacher-child interactions: Relations with children`s self-concept in second grade. Infant and Child Development, 19, 385-405. doi: $10.1002 /$ icd.672

Liebers, K. (2008). Überblick zum Forschungsstand Schulanfang. [A review on the state of research concerning school start]. In K. Liebers (Ed.), Kinder in der flexiblen Schuleingangsphase. Perspektiven für einen gelingenden Schulstart (pp. 102-140). Wiesbaden, Germany: Verlag für Sozialwissenschaften.

Lillard, A. (2012). Preschool children's development in classic Montessori, supplemented Montessori, and conventional programs. Journal of School Psychology, 50, 379-401.

Lillard, A., \& Else-Quest, N. (2006). Evaluating montessori education. Science, 313, 18931894. doi: $10.1126 /$ science. 1132362

Marsh, H. W., \& Martin, A. J. (2011). Academic self-concept and academic achievement: Relations and causal ordering. British Journal of Educational Psychology, 81, 59-77. doi: 10.1348/000709910X503501

Marsh, H. W., \& Parker, J. W. (1984). Determinants of student self-concept - Is it better to be a relatively large fish in a small pond even if you don't learn to swim as well. Journal of Personality and Social Psychology, 47, 213-231. doi: 10.1037/0022-3514.47.1.213

May, P. (2002). Hamburger Schreib-Probe (HSP) 1-9 (6th ed.). [Hamburg spelling test]. Hamburg, Germany: vpm.

Mayringer, H., \& Wimmer, H. (2005). Salzburger Lese-Screening für die Klassenstufen 1-4 (SLS) (2nd ed.). [Salzburg reading screening for 1 to 4th grade]. Bern, Switzerland: Hans Huber.

Michel, E., Kauer, M., \& Roebers, C. M. (2011). Motorische Koordinationsdefizite im Kindesalter: Welche Bedeutungen haben kognitive Basisfunktionen? [Motor coordination deficits in childhood: The role of basic cognitive processes]. Kindheit und Entwicklung, 20, 49-58. doi: 10.1026/0942-5403/a000024 
Miyake, A., Friedman, N. P., Emerson, M. J., Witzki, A. H., Howerter, A., \& Wager, T. D. (2000). The unity and diversity of executive functions and their contribution to complex frontal lobe tasks: A latent variable analysis. Cognitive Psychology, 41, 49100. doi: 10.1006/cogp.1999.0734

Moffitt, T. E., Arseneault, L., Belsky, D., Dickson, N., Hancox, R. J., Harrington, H., . . . \& Caspi, A. (2011). A gradient of childhood self-control predicts health, wealth, and public safety. PNAS Proceedings of the National Academy of Sciences of the United States of America, 108, 2693-2698. doi:10.1073/pnas.1010076108

Moser, U., \& Bayer, N. (2010). Schlussbericht der summativen Evaluation: Lernfortschritte vom Eintritt ind die Eingangsstufe bis zum Ende der dritten Klasse der Primarschule. EDK-Ost: Schulverlag Plus AG.

Nelson, T. O., \& Narens, L. (1990). A theoretical framework and new findings. In G. Bower (Ed.), The psychology of learning and motivation: Advances in research and theory (Vol. 26, pp. 125-141). New York, NY: Academic Press.

Neuenschwander, R., Röthlisberger, M., Michel, E., \& Roebers, C. M. (2011). Unterschiede in ausgewählten Bereichen der Schulfähigkeit: ein Vergleich von Kindergarten und einem neuen Schuleingangsmodell in der Schweiz. [Differences in selected aspects of school readiness: A systematic comparison of kindergarten and an innovative school transition project]. Psychologie in Erziehung und Unterricht, 58, 30-40.

Nicholls, J. G. (1978). The development of the concepts of effort and ability, perception of academic attainment, and the understanding that difficult tasks require more ability. Child Development, 49, 800-814.

Nurmi, J.-E., \& Aunola, K. (2005). Task-motivation during the first school years: A personoriented approach to longitudinal data. Learning and Instructions, 15, 103-122. doi: 10.1016/j.learninstruc.2005.04.009

OECD. (2001). Bildung auf einen Blick 2001. Paris: OECD Publishing. 
OECD. (2005). Pisa 2003: Technical Report. Paris: OECD.

OECD. (2006). Ein guter Start ins Leben II: Frühkindliche Betreuung, Bildung und Erziehung OECD Multilingual Summaries. Paris: OECD.

Perry, N. E. (1998). Young children's self-regulated learning and contexts that support it. Journal of Educational Psychology, 90, 715-729.

Pressley, M., Borkowski, J. G., \& Schneider, W. (1989). Good information processing: What it is and how education can promote it. International Journal of Educational Research, 13, 857-867. doi:10.1016/0883-0355(89)90069-4

Roebers, C. M., Cimeli, P., Röthlisberger, M., \& Neuenschwander, R. (in press). Executive functioning, metacognition, and self-perceived competence in elementary school children: An explorative study on their interrelations and their role for school achievement. Metacognition \& Learning.

Roebers, C. M., \& Fernandez, O. (2002). The effects of accuracy motivation on children's and adults' event recall, suggestibility, and their answers to unanswerable questions. Journal of Cognition and Development, 3, 415-443. doi: 10.1207/S15327647JCD3,403

Roebers, C. M., \& Kauer, M. (2009). Motor and cognitive control in a normative sample of 7year-olds. Developmental Science, 12, 175-181. doi: 10.1111/j.14677687.2008.00755.x

Roebers, C. M., Röthlisberger, M., Cimeli, P., Michel, E., \& Neuenschwander, R. (2011). School enrollment and executive functioning: A longitudinal perspective on developmental progression, stability, the influence of learning context and the prediction of preacademic skills. European Journal of Developmental Psychology, 8 , 526-540. doi: 10.1080/17405629.2011.571841 
Roebers, C. M., Schmid, C., \& Roderer, T. (2009). Metacognitive monitoring and control processes involved in primary school children's test performance. British Journal of Educational Psychology, 79, 749-767. doi: 10.1348/978185409X429842

Roebers, C. M., \& Schneider, W. (1999). Self-concept and anxiety in immigrant children. International Journal of Behavioral Development, 23, 125-147. doi: $10.1080 / 016502599384035$

Röthlisberger, M., \& Michel, E. (2009). Entwicklung und Evaluation eines Programms zur koordinativen Förderung von Kindern in Einschulungsklassen. Praxis der Kinderpsychologie und Kinderpsychiatrie, 58, 215-230.

Röthlisberger, M., Neuenschwander, R., Cimeli, P., Michel, E. \& Roebers, C. (2012). Improving children`s executive functions in 5- and 6-year-olds: Evaluation of a small group intervention in prekindergarten and kindergarten children. Infant and Child Development, 21, 411-429. DOI: 10.1002/icd.752

Sammons, P., Elliot, K., Sylva, K., Melhuish, E., \& Taggart, B. (2004). The impact of preschool on young children's cognitive attainments at entry to reception. British Educational Research Journal, 30, 691-712.

Schmid, C., Zoelch, C., \& Roebers, C. M. (2008). Das Arbeitsgedächtnis von 4- bis 5jährigen Kindern.[Working memory in 4- to 5-year-old children]. Zeitschrift für Entwicklungspsychologie und Pädagogische Psychologie, 40, 2-12.

Schneider, W. (2010). Metacognition and memory development in childhood and adolescence. In H. Salaters Waters \& W. Schneider (Eds.), Metacognition, strategy use, and instruction (pp. 54-84). New York, NY: Guilford Press.

Schneider, W., \& Lockl, K. (2008). Procedural metacognition in children: evidence for developmental trends. In J. Dunlosky \& R. A. Bjork (Eds.), Handbook of metamemory and memory (pp. 391-409). New York, NY: Lawrence Erlbaum. 
Skibbe, L. E., Connor, C. M., Morrison, F. J., \& Jewkes, A. M. (2011). Schooling effects on preschoolers' self-regulation, early literacy, and language growth. Early Childhood Research Quarterly, 26, 42-49. doi: 10.1016/j.ecresq.2010.05.001

Spinath, B., Spinath, F. M., Harlaar, N., \& Plomin, R. (2006). Predicting school achievement from general cognitive ability, self-perceived ability, and intrinsic value. Intelligence, 34, 363-374. doi: 10.1016/j.intell.2005.11.004

Stipek, D. J., \& Daniels, D. H. (1988). Declining perceptions of competence: A consequence of changes in the child or in the educational environment? Journal of Educational Psychology, 80, 352-356. doi: 10.1037/0022-0663.80.3.352

Stipek, D. J., Feiler, R., Daniels, D., \& Milburn, S. (1995). Effects of different instructional approaches on young children`s achievement and motivation. Child Development, 66, 209-223. doi: 10.1111/j.1467-8624.1995.tb00866.x

Stipek, D. J., Feiler, R., Byler, P., Ryan, R., Milburn, S., \& Salmon, J. M. (1998). Good beginnings: What difference does the program make in preparing young children for school? Journal of Applied Developmental Psychology, 19, 41-66. doi: $10.1016 / \mathrm{S} 0193-3973(99) 80027-6$

Sylva, K., Taggart, B., Siraj-Blatchford, I., Totsika, V., Ereky-Stevens, K., Gildena, R., \& Bell, D. (2007). Curricular quality and day-to-day learning activities in pre-school. International Journal of Early Years Education, 15, 49-64.

Thorell, L. B., Lindqvist, S., Nutley, S. B., Bohlin, G., \& Klingberg, T. (2009). Training and transfer effects of executive functions in preschool children. Developmental Science, 12, 106-113. doi: 10.1111/j.1467-7687.2008.00745.x

Vogt, F., Zumwald, B., Urech, C., \& Abt, N. (2010). Schlussbericht der formativen Evaluation. Grund-/Basisstufe: Umsetzung, Unterrichtsentwicklung und Akzeptanz bei Eltern und Lehrpersonen (pp. 24-44). EDK-Ost: Schulverlag Plus AG. 
Winne, P. H. \& Hadwin (2008). Self-regulated learning viewed from models of information processing. In B. J. Zimmerman \& D. H. Schunk (Eds.), Self-regulated learning and academic achievement: Theoretical perspectives (pp. 153-190). Mahwah, NJ: Lawrence Erlbaum.

Zimmerman, B. J., \& Moylan, A. R. (2009). Where metacognition and motivation intersect. In D. J. Hacker (Ed.), Handbook of metacognition in education (pp. 299-316). New York, NY: Taylor \& Francis. 


\section{Table 1}

Characteristics of the Matched Sample at the beginning of the study

\begin{tabular}{|c|c|c|c|}
\hline & $\begin{array}{c}\text { Traditional School } \\
\text { System }\end{array}$ & $\begin{array}{c}\text { Mixed-Grades } \\
\text { Classes }\end{array}$ & Min - Max \\
\hline \multicolumn{4}{|l|}{ Younger Age Group } \\
\hline $\mathrm{N}$ & 70 & 70 & \\
\hline Sex (female, \%) & 45.7 & 45.7 & \\
\hline Age (in Months) & $68.4(3.7)$ & $69.3(5.0)$ & $61-78$ \\
\hline Language Skills & $95.6(13.9)$ & $94.8(16.0)$ & $58-132$ \\
\hline Speed of Processing & $440.0(76.8)$ & $421.3(73.2)$ & $287-659$ \\
\hline Non-verbal Intelligence & $4.9(2.0)$ & $5.1(2.0)$ & $0-11$ \\
\hline SES & $8.6(3.3)$ & $8.8(3.5)$ & $4-19$ \\
\hline \multicolumn{4}{|l|}{ Older Age Group } \\
\hline $\mathrm{N}$ & 45 & 45 & \\
\hline Sex (female, \%) & 66.7 & 66.7 & \\
\hline Age (in Months) & $78.2(3.7)$ & $79.7(4.2)$ & $66-85$ \\
\hline Language Skills & $97.4(9.2)$ & $97.1(9.9)$ & $69-121$ \\
\hline Speed of Processing & $386.1(67.7)$ & $394.6(70.7)$ & $271.8-618.8$ \\
\hline Non-verbal Intelligence & $6.6(1.9)$ & $6.6(2.1)$ & $2-10$ \\
\hline SES & $9.7(2.9)$ & $10.6(2.9)$ & $5-18$ \\
\hline
\end{tabular}

Note. $N=230$ children who completed $\mathrm{T}_{1}, \mathrm{~T}_{2}$ and $\mathrm{T}_{3}$ and who are included in the present paper; Language Skills: Verbal IQ-score; Speed of Processing: Median reaction time in msec; Non-verbal Intelligence: raw scores; SES: Prestige Points of Parental Education and Current Profession 


\section{Table 2}

Partial correlations (controlling for chronological age in months) between the included student's characteristics; partial correlations for the traditional school system before the slash (/), for the mixed-grades classes behind the slash

\begin{tabular}{l|cccc}
\hline & 2. & 3. & 4. & 5. \\
\hline 1. Mathematic & $.66^{* *} / .59^{* *}$ & $.10 / .15$ & $.38^{* *} / .28^{* *}$ & $.24 * / .28^{*}$ \\
\multicolumn{1}{l}{ Achievement } & -- & $.15 / .25^{* *}$ & $.30^{* *} / .21^{*}$ & $.41^{* *} / .49^{* *}$ \\
2. Literacy & & -- & $.11 / .19^{*}$ & $.07 / .06$ \\
3. Academic Self-Concept & & & -- & $.11 / .08$ \\
4. Executive Functions & & & & \\
5. Metacognition & & & & \\
\hline
\end{tabular}

Note: $* p<.01 ; * * p<.001$ 


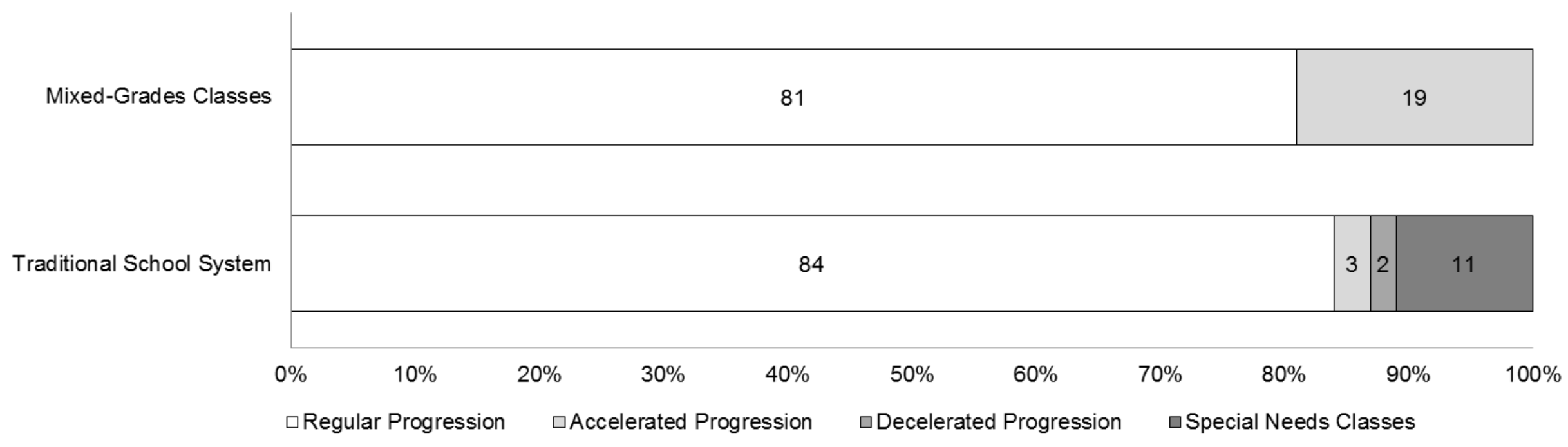

Figure 1. Young students`school progression as a function of school context 


\section{Early Literacy}
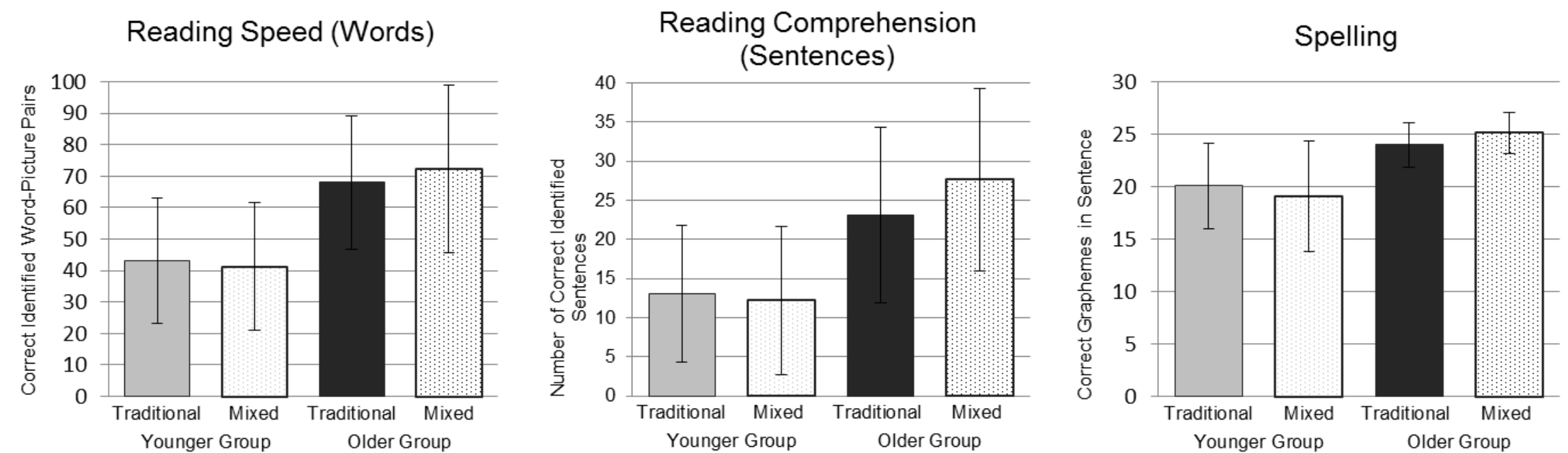

Figure 2. Means and standard deviations in early literacy (at measurement point 3 ) in the two age groups and the two school systems (traditional $=$ traditional school system, mixed $=$ mixed-grades classes $)$. 


\section{Early Mathematical Skills}
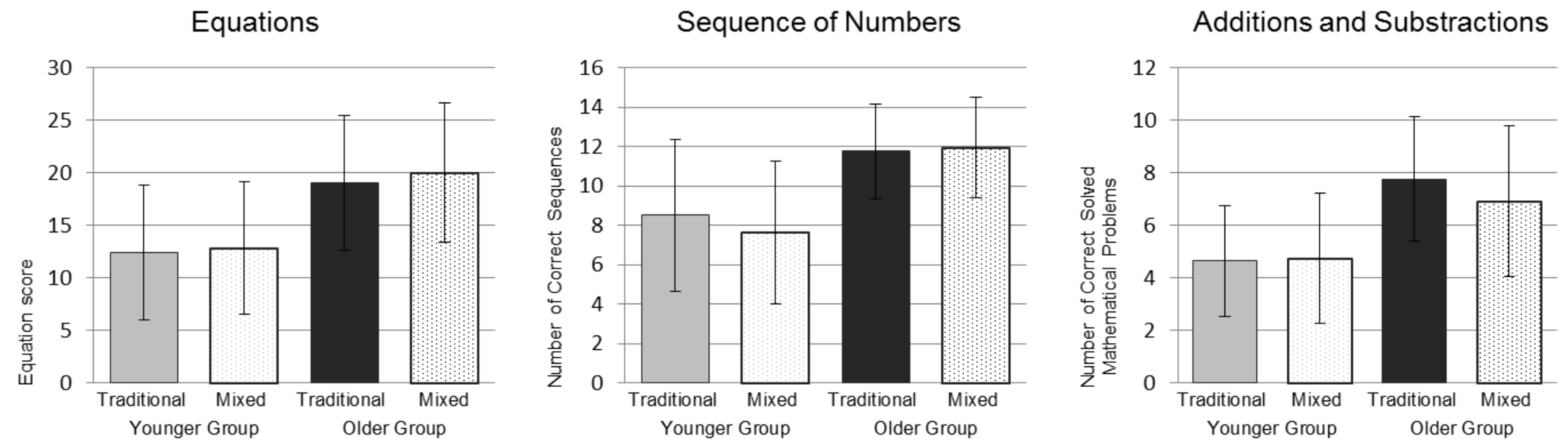

Figure 3. Means and standard deviations in early mathematical skills (at measurement point 3) as a function of age group and school system $($ traditional $=$ traditional school system, mixed $=$ mixed-grades classes $)$. 

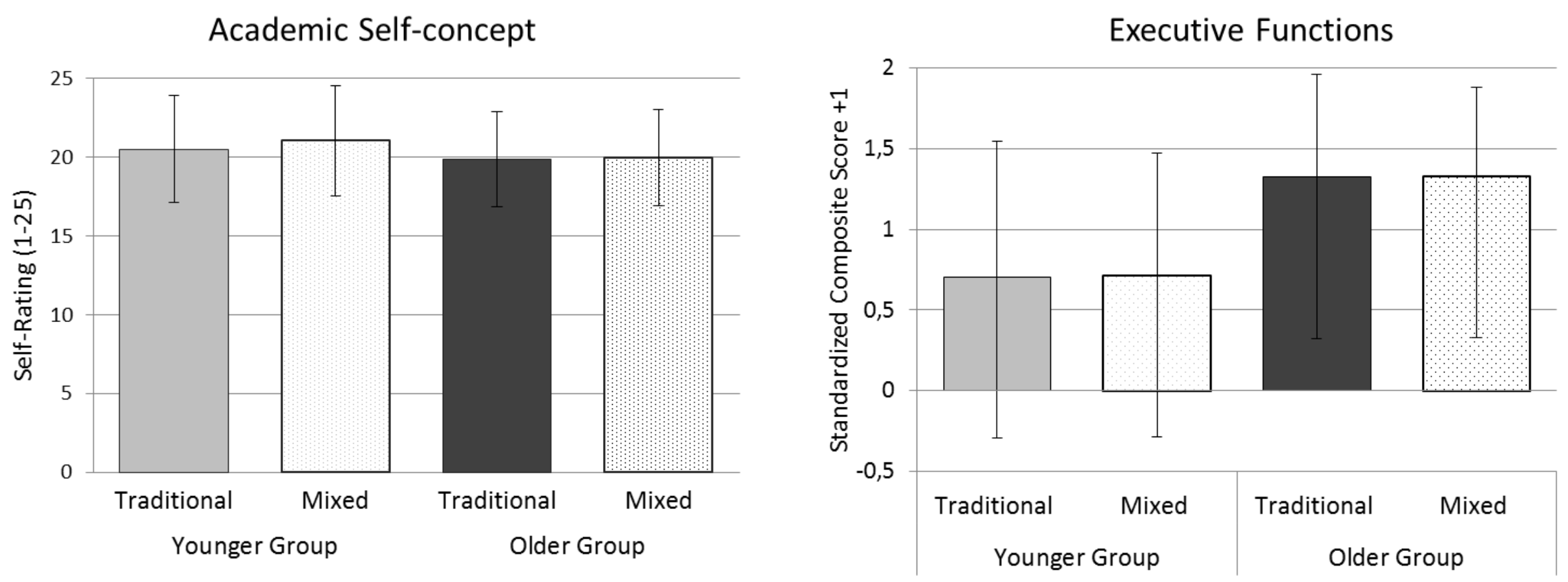

Figure 4: Children's academic self-concept (left panel; embracing mathematics and literacy) and executive functions (right panel) as a function of age group and school context 


\section{Metacognition}
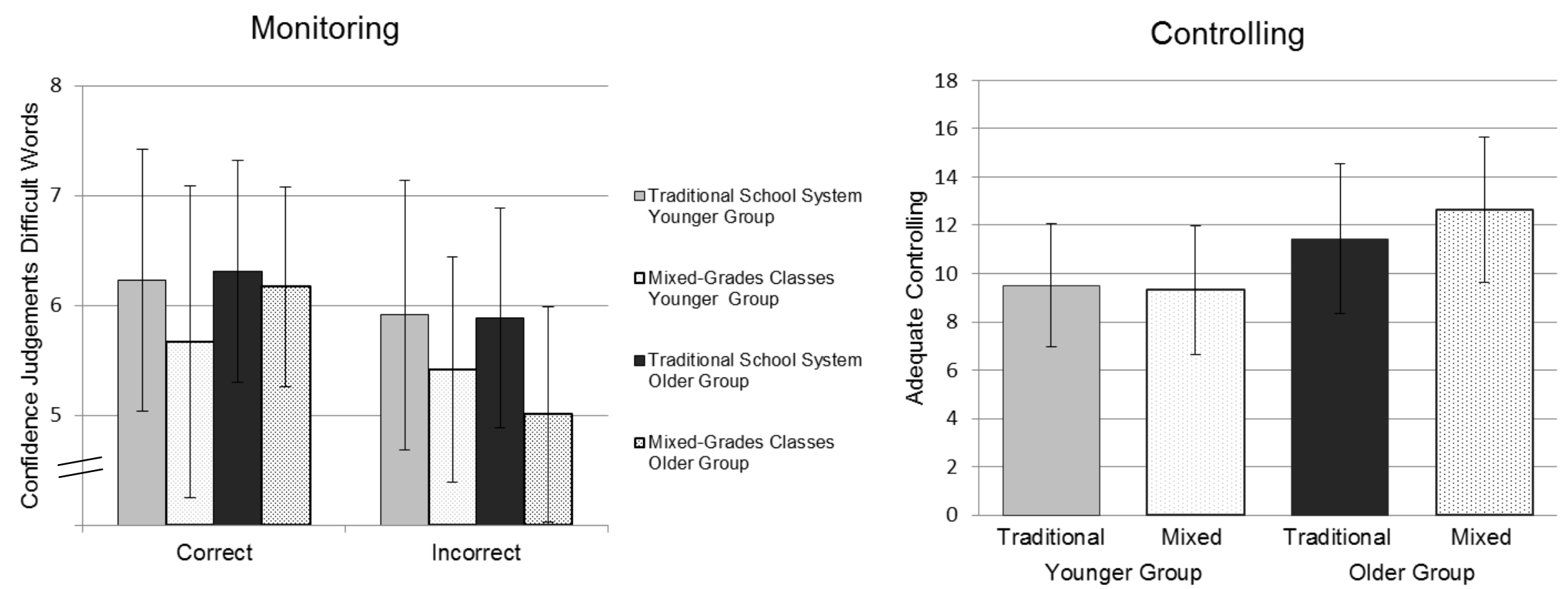

Figure 5. Means and standard deviations in monitoring (confidence judgments for correctly and incorrectly spelled difficult words) and in controlling (adequately withdrawn and maintained words) in the two age groups and the two school systems (at measurement point 3), traditional= traditional school system, mixed= mixed-grades classes. 